\title{
THE GROUPOID APPROACH TO LEAVITT PATH ALGEBRAS
}

\author{
SIMON W. RIGBY
}

\begin{abstract}
When the theory of Leavitt path algebras was already quite advanced, it was discovered that some of the more difficult questions were susceptible to a new approach using topological groupoids. The main result that makes this possible is that the Leavitt path algebra of a graph is graded isomorphic to the Steinberg algebra of the graph's boundary path groupoid.

This expository paper has three parts: Part 1 is on the Steinberg algebra of a groupoid, Part 2 is on the path space and boundary path groupoid of a graph, and Part 3 is on the Leavitt path algebra of a graph. It is a self-contained reference on these topics, intended to be useful to beginners and experts alike. While revisiting the fundamentals, we prove some results in greater generality than can be found elsewhere, including the uniqueness theorems for Leavitt path algebras.
\end{abstract}

Leavitt path algebras are $\mathbb{Z}$-graded algebras with involution, whose generators and relations are encoded in a directed graph. Steinberg algebras, on the other hand, are algebras of functions defined on a special kind of topological groupoid, called an ample groupoid. To understand how they are related, it is useful to weave together some historical threads. This historical overview might not be comprehensive, but it is intended to give some idea of the origins of our subject.

0.1. Historical overview: Groupoids, graphs, and their algebras. In the late 1950s and early 1960s, William G. Leavitt [51, 52] showed that there exist simple rings whose finite-rank free modules admit bases of different sizes. In a seemingly unrelated development, in 1977, Joachim Cuntz [33] showed that there exist separable $C^{*}$-algebras that are simple and purely infinite. Cuntz's paper was one of the most influential in the history of operator theory. It provoked intense interest (that is still ongoing) in generalising, classifying, and probing the structure of various classes of $C^{*}$-algebras. One of the next landmarks was reached in 1980, when Jean Renault [59] defined groupoid $C^{*}$-algebras, taking inspiration from the $C^{*}$-algebras that had previously been associated to transformation groups. The Cuntz algebras were interpreted as groupoid $C^{*}$-algebras, and from that point onwards there was a new framework and some powerful results with which to pursue new and interesting examples.

In 1997, Kumjian, Pask, Raeburn, and Renault [50] showed how to construct a Hausdorff ample groupoid (and hence a $C^{*}$-algebra) from a row- and column-finite directed graph with no sinks. They showed that these $C^{*}$-algebras universally satisfy the Cuntz-Krieger relations from [34], which had become significant in the intervening years. Graph $C^{*}$-algebras were then studied in depth. Usually, they were conceptualised in terms of the partial isometries that generate them; direct methods, rather than groupoid methods, were used predominantly $[16,58]$. Meanwhile, the Cuntz algebras had also been interpreted as inverse semigroup $C^{*}$-algebras. Paterson [56,57], at the turn of the 21st century, organised the situation a bit better. He showed that all graph $C^{*}$-algebras (of countable graphs, possibly with sinks, infinite emitters, and infinite receivers) are inverse semigroup $C^{*}$-algebras, and that all inverse semigroup $C^{*}$-algebras are groupoid $C^{*}$-algebras. The key innovation was defining the universal groupoid of an inverse semigroup, which is an ample but not necessarily Hausdorff topological groupoid.

It is unclear when the dots were first connected between Leavitt's algebras and Cuntz's $C^{*}$-algebras (probably in [12], a very long time after they first appeared). The Cuntz algebra $\mathcal{O}_{n}$ is the norm completion of the complex Leavitt algebra $L_{n, \mathbb{C}}$. Over any field $\mathbb{K}$, the ring $L_{n, \mathbb{K}}$ and the $C^{*}$-algebra $\mathcal{O}_{n}$ are purely infinite simple, and they have the same $K_{0}$ group (but these concepts have a different meaning for rings compared to $C^{*}$-algebras). This begins a process in which the algebraic community generalises, classifies, and probes the structure of various classes of rings in much the same way as the operator algebra community did with $C^{*}$-algebras. Leavitt path algebras were introduced in [3] and [13] 
as universal $\mathbb{K}$-algebras satisfying path algebra relations and Cuntz-Krieger relations. Generalising the relationship between the Leavitt and Cuntz algebras, the graph $C^{*}$-algebra of a graph $E$ is the norm completion of the complex Leavitt path algebra of $E$. The interplay with $C^{*}$-algebras is not the only connection between Leavitt path algebras and other, older, areas of mathematics - see for instance [1, $\S 1]$ and [55].

Knowing what we know now, the next step was very natural. Is there a way of defining a "groupoid $\mathbb{K}$-algebra" in such a way that:

- When the input is the universal groupoid of an inverse semigroup $S$, the output is the (discrete) inverse semigroup algebra $\mathbb{K} S$;

- When the input is a graph groupoid $\mathcal{G}_{E}$, the output is the Leavitt path algebra $L_{\mathbb{K}}(E)$ ?

This question was asked and answered by Steinberg [63], and Clark, Farthing, Sims, and Tomforde [27]. Consistent with previous experiences of converting operator algebra constructions into $\mathbb{K}$-algebra constructions, they found that the groupoid $C^{*}$-algebra is the norm completion of the groupoid $\mathbb{C}$-algebra. It is also worth noting that Steinberg chose a broad scope and defined groupoid $R$-algebras over any commutative ring $R$, rather than just fields. We call these groupoid algebras Steinberg algebras.

Each of the three parts in this paper can be read separately. However, we work towards Leavitt path algebras as the eventual subject of interest, and this influences the rest of the text. For example, in Part 1 we try not to impose the Hausdorff assumption on groupoids if it is not necessary, but there are no examples here of non-Hausdorff groupoids. Throughout, we use the graph theory notation and terminology that is conventional in Leavitt path algebras. (In most of the $C^{*}$-algebra literature, the orientation of paths is reversed.) And in Part 2, we ignore some topics like amenability that would be important if we were intending to study the $C^{*}$-algebras of boundary path groupoids.

A standing assumption throughout the paper is that $R$ is a commutative ring with 1 . We rarely need to draw attention to it or require it to be anything special.

0.2. Background: Leavitt path algebras. For an arbitrary graph $E$, there is an $R$-algebra, $L_{R}(E)$, called the Leavitt path algebra of $E$. The role of the graph may seem unclear at the outset, because all it does is serve as a kind of notational device for the generators and relations that define $L_{R}(E)$. Surprisingly, it turns out that many of the ring-theoretic properties of $L_{R}(E)$ are controlled by graphical properties of $E$. For example, the Leavitt path algebra has some special properties if the graph is acyclic, cofinal, downward-directed, has no cycles without exits, etc.

Since 2005, there has been an abundance of research on Leavitt path algebras. One of the main goals has been to characterise their internal properties, ideals, substructures, and modules. As a result, we have a rich supply of algebras with "interesting and extreme properties" [8]. This is useful for generating counterexamples to reasonable-sounding conjectures, e.g. [6, 46], or for supporting other long-standing conjectures by showing they hold within this varied class, e.g. [9, 15].

Another goal has been finding invariants that determine Leavitt path algebras up to isomorphism, or Morita equivalence. This enterprise is known as the classification question for Leavitt path algebras. Of course, something that is easier than classifying all Leavitt path algebras is classifying those that have a certain property (like purely infinite simplicity), or classifying the Leavitt path algebras of small graphs. This has led to interesting developments in $K$-theory (see $[2, \S 6.3]$ and [44]) and has motivated the study of substructures of Leavitt path algebras, like the socle [14] and invariant ideals [47].

A third goal is to explain why graph $C^{*}$-algebras and Leavitt path algebras have so much in common. One expects a priori that these two different structures would have little to do with one another. But in fact, many theorems about Leavitt path algebras resemble theorems about graph $C^{*}$-algebras [1, Appendix 1]. For instance, the graphs whose $C^{*}$-algebras are $C^{*}$-simple are exactly the same graphs whose Leavitt path algebras are simple (over any base field). One conjecture in this general direction is the Isomorphism Conjecture for Graph Algebras [7]: if $E$ and $F$ are two graphs such that $L_{\mathbb{C}}(E) \cong L_{\mathbb{C}}(F)$ 
as rings, then $C^{*}(E) \cong C^{*}(F)$ as $*$-algebras. In the unital case, an affirmative answer has been given in [37, Theorem 14.7].

0.3. Background: Steinberg algebras. An ample groupoid is a special kind of locally compact topological groupoid. The Steinberg algebra of such a groupoid is an $R$-module of functions defined on it. It becomes an associative $R$-algebra once it is equipped with a generally noncommutative operation called the convolution (generalising the multiplicative operation on a group algebra). If the groupoid $\mathcal{G}$ is Hausdorff, one can characterise its Steinberg algebra quite succinctly as the convolution algebra of locally constant, compactly supported functions $f: \mathcal{G} \rightarrow R$. Steinberg algebras first appeared independently in [63] and [27]. The primary motivation was to generalise other classes of algebras, especially inverse semigroup algebras and Leavitt path algebras.

Steinberg algebras do not only unify and generalise some seemingly disparate classes of algebras, but they also provide an entirely new approach to studying them. Many theorems about Leavitt path algebras and inverse semigroup algebras have since been recovered as specialisations of more general theorems about Steinberg algebras. For example, various papers [28, 64, 65, 67] have used groupoid techniques to characterise, in terms of the underlying graph or inverse semigroup, when a Leavitt path algebra or inverse semigroup algebra is (semi)prime, indecomposable, (semi)primitive, noetherian, or artinian.

Simplicity theorems play a very important role in graph algebras and some related classes of algebras. (In contrast, inverse semigroup algebras are never simple.) This theme goes right back to the beginning, when Leavitt proved in [52] that the Leavitt algebras $L_{n, \mathbb{K}}(n \geq 2)$ are all simple. Likewise, Cuntz proved in [33] that the Cuntz algebras $\mathcal{O}_{n}(n \geq 2)$ are $C^{*}$-simple in the sense that they have no closed two-sided ideals. When Leavitt path algebras were introduced, in the very first paper on the subject, Abrams and Aranda Pino [3] wrote the simplicity theorem for Leavitt path algebras of row-finite graphs. It was extended to Leavitt path algebras of arbitrary graphs, as soon as these were defined in [4].

Once Steinberg algebras appeared on the scene, Brown, Clark, Farthing, and Sims [19] proved a simplicity theorem for Steinberg algebras of Hausdorff ample groupoids over $\mathbb{C}$. That effort led them to unlock a remarkable piece of research in which they derived a simplicity theorem for the $C^{*}$-algebras of secondcountable, locally compact, Hausdorff étale groupoids. It speaks to the significance of these new ideas, that they were put to use in solving a problem that was open for many decades. The effort has recently been repeated for non-Hausdorff groupoids, in [26], where it is said that "We view Steinberg algebras as a laboratory for finding conditions to characterize $C^{*}$-simplicity for groupoid $C^{*}$-algebras."

Besides the ones we have already discussed, there are many interesting classes of algebras that appear as special cases of Steinberg algebras. These include partial skew group rings associated to topological partial dynamical systems [17], and Kumjian-Pask algebras associated to higher-rank graphs [31]. In quite a different application, Nekrashevych [54] has produced Steinberg algebras with prescribed growth properties, including the first examples of simple algebras of arbitrary Gelfand-Kirrilov dimension.

0.4. Background: Graph groupoids. There are actually a few ways to associate a groupoid to a graph $E$; see for example [50, p. 511], [56, pp. 156-159], and [30, Example 5.4]. The one that we are interested in is called the boundary path groupoid, $\mathcal{G}_{E}$. Its unit space is the set of all paths that are either infinite or end at a sink or an infinite emitter (i.e., boundary paths). This groupoid was introduced in its earliest form, for row- and column-finite graphs without sinks, by Kumjian, Pask, Raeburn, and Renault [50]. It bears a resemblance to a groupoid studied a few years earlier by Deaconu [35]. The construction was later generalised in a number of different directions, taking a route through inverse semigroup theory [57], and going as far as topological higher-rank graphs (e.g. [49, 61, 71]).

The boundary path groupoid is an intermediate step towards proving that all Leavitt path algebras are Steinberg algebras, and it becomes an important tool for the analysis of Leavitt path algebras. For an arbitrary graph $E$, there is a $\mathbb{Z}$-graded isomorphism $A_{R}\left(\mathcal{G}_{E}\right) \cong L_{R}(E)$, where $A_{R}\left(\mathcal{G}_{E}\right)$ is the Steinberg algebra of $\mathcal{G}_{E}$ and $L_{R}(E)$ is the Leavitt path algebra of $E$. Consequently, if we understand some property of Steinberg algebras (for example, the centre [29]) then we can understand that property of Leavitt path algebras by translating groupoid terms into graphical terms and applying the isomorphism 
$A_{R}\left(\mathcal{G}_{E}\right) \cong L_{R}(E)$. Similarly, there is an isometric $*$-isomorphism $C^{*}\left(\mathcal{G}_{E}\right) \cong C^{*}(E)$, where $C^{*}\left(\mathcal{G}_{E}\right)$ is the full groupoid $C^{*}$-algebra of $\mathcal{G}_{E}$ and $C^{*}(E)$ is the graph $C^{*}$-algebra of $E$.

The diagonal subalgebra of a Steinberg algebra (resp., groupoid $C^{*}$-algebra) is the commutative subalgebra (resp., $C^{*}$-subalgebra) generated by functions supported on the unit space. If two ample groupoids $\mathcal{F}$ and $\mathcal{G}$ are topologically isomorphic, it is immediate that $A_{R}(\mathcal{F}) \cong A_{R}(\mathcal{G})$ and the isomorphism sends the diagonal to the diagonal. The converse is a very interesting and current research topic called "groupoid reconstruction". It was shown in [11] that if $\mathcal{F}$ and $\mathcal{G}$ are topologically principal, and $R$ is an integral domain, then $A_{R}(\mathcal{F}) \cong A_{R}(\mathcal{G})$ with an isomorphism that preserves diagonals if and only if $\mathcal{F} \cong \mathcal{G}$. This was generalised in [23] and [66]. For $C^{*}$-algebras, there are results of a similar flavour [60] .

For boundary path groupoids, groupoid reconstruction is essentially the question: if $E$ and $F$ are graphs such that $L_{R}(E) \cong L_{R}(F)$, does it imply $\mathcal{G}_{E} \cong \mathcal{G}_{F}$ ? Many mathematicians [11, 20, 22, 66] have been working on this and they have given positive answers after imposing various assumptions on the graphs, the ring $R$, or the type of isomorphism between the Leavitt path algebras. It seems likely that more results will emerge. It is already known from [21, Theorem 5.1] that if there exists a diagonal-preserving isomorphism of graph $C^{*}$-algebras $C^{*}(E) \cong C^{*}(F)$, then $\mathcal{G}_{E} \cong \mathcal{G}_{F}$. It is plausible that the groupoid reconstruction programme for graph groupoids could eventually prove the general Isomorphism Conjecture for Graph Algebras [7].

\section{The Steinberg ALGeBra of A Groupoid}

Part 1 is structured as follows. It begins, in $\S 1.1$ by providing some background on groupoids. In $\S 1.2$, we develop some facts about topological groupoids and almost immediately specialise to étale and ample groupoids. We give a very brief treatment of inverse semigroups and their role in the subject. In $\S 1.3$, we introduce the Steinberg algebra of an ample groupoid, describing it in a few different ways to make the definition more transparent. We develop the basic theory in a self-contained way, paying attention to what can and cannot be said about non-Hausdorff groupoids. In $\S 1.4$, we investigate some important properties, showing that these algebras are locally unital and enjoy a kind of symmetry that comes from an involution (in other words, they are $*$-algebras). In $\S 1.5$, we investigate the effects of groupoid-combining operations like products, disjoint unions, and directed unions, and find applications with finite-dimensional Steinberg algebras and the Steinberg algebras of approximately finite groupoids. In $§ 1.6$, we discuss graded groupoids and graded Steinberg algebras.

1.1. Groupoids. This classical definition of a groupoid is modified from [59]. We have chosen to paint a complete picture; indeed, some parts of the definition can be derived from other parts.

Definition 1.1. A groupoid is a $\operatorname{system}\left(\mathcal{G}, \mathcal{G}^{(0)}, \boldsymbol{d}, \boldsymbol{c}, \boldsymbol{m}, \boldsymbol{i}\right)$ such that:

(G1) $\mathcal{G}$ and $\mathcal{G}^{(0)}$ are nonempty sets, called the underlying set and unit space, respectively;

(G2) $\boldsymbol{d}, \boldsymbol{c}$ are maps $\mathcal{G} \rightarrow \mathcal{G}^{(0)}$, called domain and codomain;

(G3) $\boldsymbol{m}$ is a partially defined binary operation on $\mathcal{G}$ called composition: specifically, it is a map from the set of composable pairs

$$
\mathcal{G}^{(2)}=\{(g, h) \in \mathcal{G} \times \mathcal{G} \mid \boldsymbol{d}(g)=\boldsymbol{c}(h)\}
$$

onto $\mathcal{G}$, written as $\boldsymbol{m}(g, h)=g h$, with the properties:

- $\boldsymbol{d}(g h)=\boldsymbol{d}(h)$ and $\boldsymbol{c}(g h)=\boldsymbol{c}(g)$ whenever the composition $g h$ is defined;

- $(g h) k=g(h k)$ whenever either side is defined;

(G4) For every $x \in \mathcal{G}^{(0)}$ there is a unique identity $1_{x} \in \mathcal{G}$ such that $1_{x} g=g$ whenever $\boldsymbol{c}(g)=x$, and $h 1_{x}=h$ whenever $\boldsymbol{d}(h)=x$; 
(G5) $\boldsymbol{i}: \mathcal{G} \rightarrow \mathcal{G}$ is a map called inversion, written as $\boldsymbol{i}(g)=g^{-1}$, such that $g^{-1} g=1_{\boldsymbol{c}(g)}, g g^{-1}=1_{\boldsymbol{d}(g)}$, and $\left(g^{-1}\right)^{-1}=g$.

The definition can be summarised by saying: a groupoid is a small category in which every morphism is invertible. Having said this, the elements of $\mathcal{G}$ will usually be called morphisms.

Remark 1.2. We always identify $x \in \mathcal{G}^{(0)}$ with $1_{x} \in \mathcal{G}$, so $\mathcal{G}^{(0)}$ is considered a subset of $\mathcal{G}$. The elements of $\mathcal{G}^{(0)}$ are called units.

Many authors write $\boldsymbol{s}$ (source) and $\boldsymbol{r}$ (range) instead of $\boldsymbol{d}$ and $\boldsymbol{c}$ in the definition of a groupoid. Our notation is chosen to avoid confusion in the context of graphs, where $s$ and $r$ refer to the source and range, respectively, of edges and directed paths.

A homomorphism between groupoids $\mathcal{G}$ and $\mathcal{H}$ is a functor $F: \mathcal{G} \rightarrow \mathcal{H}$; that is, a map sending units of $\mathcal{G}$ to units of $\mathcal{H}$ and mapping all the morphisms in $\mathcal{G}$ to morphisms in $\mathcal{H}$ in a way that respects the structure. A subgroupoid is a subset $\mathcal{S} \subseteq \mathcal{G}$ that is a groupoid with the structure that it inherits from $\mathcal{G}$. For $x \in \mathcal{G}^{(0)}$, we use the notation $x \mathcal{G}=\boldsymbol{c}^{-1}(x), \mathcal{G} x=\boldsymbol{d}^{-1}(x)$, and $x \mathcal{G} y=\boldsymbol{c}^{-1}(x) \cap \boldsymbol{d}^{-1}(y)$. The set $x \mathcal{G} x$ is a group, called the isotropy group based at $x$, and the set $\operatorname{Iso}(\mathcal{G})=\bigcup_{x \in \mathcal{G}^{(0)}} x \mathcal{G} x$ is a subgroupoid, called the isotropy subgroupoid of $\mathcal{G}$. If $\operatorname{Iso}(\mathcal{G})=\mathcal{G}^{(0)}$ then $\mathcal{G}$ is called principal. We say that $\mathcal{G}$ is transitive if for every pair of units $x, y \in \mathcal{G}^{(0)}$ there is at least one morphism in $x \mathcal{G} y$.

The conjugacy class of $g \in \operatorname{Iso}(\mathcal{G})$ is the set $\mathrm{Cl}_{\mathcal{G}}(g)=\left\{h g h^{-1} \mid h \in \mathcal{G} \boldsymbol{c}(g)\right\}$. The set of conjugacy classes partitions $\operatorname{Iso}(\mathcal{G})$. The conjugacy class of a unit is called an orbit, and the set of orbits partitions $\mathcal{G}^{(0)}$. Equivalently, the orbit of $x \in \mathcal{G}^{(0)}$ is $\mathrm{Cl}_{\mathcal{G}}(x)=\boldsymbol{c}\left(\boldsymbol{d}^{-1}(x)\right)=\boldsymbol{d}\left(\boldsymbol{c}^{-1}(x)\right)$, or the unit space of the maximal transitive subgroupoid containing $x$. A subset $U \subseteq \mathcal{G}^{(0)}$ is invariant if for all $g \in \mathcal{G}, \boldsymbol{d}(g) \in U$ implies $\boldsymbol{c}(g) \in U$, which is to say that $U$ is a union of orbits. If $x, y \in \mathcal{G}^{(0)}$ belong to the same orbit, then the isotropy groups $x \mathcal{G} x$ and $y \mathcal{G} y$ are isomorphic. In fact, there can be many isomorphisms $x \mathcal{G} x \rightarrow y \mathcal{G} y$. For every $g \in y \mathcal{G} x$ there is an "inner" isomorphism $x \mathcal{G} x \rightarrow y \mathcal{G} y$ given by $x \mapsto g x g^{-1}$. This allows us to speak of the isotropy group of an orbit.

Examples 1.3. Many familiar mathematical objects are essentially groupoids:

(a) Any group $G$ with identity $\varepsilon$ can be viewed as a groupoid with unit space $\{\varepsilon\}$. Conjugacy classes are conjugacy classes in the usual sense.

(b) If $\left\{G_{i} \mid i \in I\right\}$ is a family of groups with identities $\left\{\varepsilon_{i} \mid i \in I\right\}$, then the disjoint union $\bigsqcup_{i \in I} G_{i}$ has a groupoid structure with $\boldsymbol{d}(g)=\boldsymbol{c}(g)=\varepsilon_{i}$ for every $g \in G_{i}$. The composition, defined only for pairs $(g, h) \in \bigsqcup_{i \in I} G_{i} \times G_{i}$, is just the relevant group law. This is known as a bundle of groups. The isotropy subgroupoid of any groupoid is a bundle of groups.

(c) Let $X$ be a set with an equivalence relation $\sim$. We define the groupoid of pairs $\mathcal{G}_{X}=\{(x, y) \in$ $X \times X \mid x \sim y\}$ with unit space $X$, and view $(x, y)$ as a morphism with domain $y$, codomain $x$, and inverse $(x, y)^{-1}=(y, x)$. A pair of morphisms $(x, y),(w, z)$ is composable if and only if $y=w$, and composition is defined as $(x, y)(y, z)=(x, z)$. Every principal groupoid is isomorphic to a groupoid of pairs. If $\sim$ is the indiscrete equivalence relation (where $x \sim y$ for all $x, y \in X$ ) then $\mathcal{G}_{X}$ is called the transitive principal groupoid on $X$.

(d) Let $G$ be a group with a left action on a set $X$. There is a groupoid structure on $G \times X$, where the unit space is $\{\varepsilon\} \times X$, or simply just $X$. We understand that the morphism $(g, x)$ has domain $g^{-1} x$ and codomain $x$. Composition is defined as $(g, x)\left(h, g^{-1} x\right)=(g h, x)$, and inversion as $(g, x)^{-1}=$ $\left(g^{-1}, g^{-1} x\right)$. The isotropy group at $x$ is isomorphic to the stabiliser subgroup associated to $x$. Orbits are orbits in the usual sense, and the groupoid is transitive if and only if the action is transitive. This is called the transformation groupoid associated to the action of $G$ on $X$.

(e) The fundamental groupoid of a topological space $X$ is the set of homotopy path classes on $X$. The unit space of this groupoid is $X$ itself, and the isotropy group at $x \in X$ is the fundamental group 
$\pi_{1}(X, x)$. The groupoid is transitive if and only if $X$ is path-connected, and it is principal if and only if every path component is simply connected.

1.2. Topological groupoids. Briefly, here are some of our topological conventions. We use the word base to mean a collection of open sets, called basic open sets, that generates a topology by taking unions. A neighbourhood base is a filter for the set of neighbourhoods of a point. In this paper, the word basis is reserved for linear algebra. A compact topological space is one in which every open cover has a finite subcover, and a locally compact topological space is one in which every point has a neighbourhood base of compact sets. If $X$ and $Y$ are topological spaces, a local homeomorphism is a map $f: X \rightarrow Y$ with the property: every point in $X$ has an open neighbourhood $U$ such that $\left.f\right|_{U}$ is a homeomorphism onto an open subset of $Y$. Every local homeomorphism is open and continuous.

The definition of a topological groupoid is straightforward, but there is some inconsistency in the literature on what it means for a groupoid to be étale or locally compact. While some papers require germane conditions, our definitions are chosen to be classical and minimally restrictive. We are mainly concerned with étale and ample groupoids. Roughly speaking, étale groupoids are topological groupoids whose topology is locally determined by the unit space.

Definition 1.4. A groupoid $\mathcal{G}$ is

(a) a topological groupoid if its underlying set has a topology, and the maps $\boldsymbol{m}$ and $\boldsymbol{i}$ are continuous, with the understanding that $\mathcal{G}^{(2)}$ inherits its topology from $\mathcal{G} \times \mathcal{G}$;

(b) an étale groupoid if it is a topological groupoid and $\boldsymbol{d}$ is a local homeomorphism.

Some pleasant consequences follow from these two definitions. In any topological groupoid, $\boldsymbol{i}$ is a homeomorphism because it is a continuous involution, and $\boldsymbol{d}$ and $\boldsymbol{c}$ are both continuous because $\boldsymbol{d}(g)=$ $\boldsymbol{m}(\boldsymbol{i}(g), g)$ and $\boldsymbol{c}=\boldsymbol{d} \boldsymbol{i}$. If $\mathcal{G}$ is étale, then $\boldsymbol{d}, \boldsymbol{c}$, and $\boldsymbol{m}$ are local homeomorphisms, and $\mathcal{G}^{(0)}$ is open in $\mathcal{G}$ (the openness of $\mathcal{G}^{(0)}$ is proved from first principles in [38, Proposition 3.2]). If $\mathcal{G}$ is a Hausdorff topological groupoid, then $\mathcal{G}^{(0)}$ is closed. Indeed (and this neat proof is from [62]) if $\left(x_{i}\right)_{i \in I}$ is a net in $\mathcal{G}^{(0)}$ with $x_{i} \rightarrow g \in \mathcal{G}$, then $x_{i}=\boldsymbol{c}\left(x_{i}\right) \rightarrow \boldsymbol{c}(g)$ because $\boldsymbol{c}$ is continuous, so $g=\boldsymbol{c}(g) \in \mathcal{G}^{(0)}$ by uniqueness of limits. If $\mathcal{G}$ is any topological groupoid, the maps $\boldsymbol{d} \times \boldsymbol{c}: \mathcal{G} \times \mathcal{G} \rightarrow \mathcal{G}^{(0)} \times \mathcal{G}^{(0)}$ and $(\boldsymbol{d}, \boldsymbol{c}): \mathcal{G} \rightarrow \mathcal{G}^{(0)} \times \mathcal{G}^{(0)}$ are both continuous. If $\mathcal{G}^{(0)}$ is Hausdorff, the diagonal $\Delta=\left\{(x, x) \mid x \in \mathcal{G}^{(0)}\right\}$ is closed in $\mathcal{G}^{(0)} \times \mathcal{G}^{(0)}$; consequently, $\mathcal{G}^{(2)}=(\boldsymbol{d} \times \boldsymbol{c})^{-1}(\Delta)$ is closed in $\mathcal{G} \times \mathcal{G}$ and $\operatorname{Iso}(\mathcal{G})=(\boldsymbol{d}, \boldsymbol{c})^{-1}(\Delta)$ is closed in $\mathcal{G}$.

Let $\mathcal{G}$ be a topological groupoid. If $U \subseteq \mathcal{G}$ is an open set such that $\left.\boldsymbol{c}\right|_{U}$ and $\left.\boldsymbol{d}\right|_{U}$ are homeomorphisms onto open subsets of $\mathcal{G}^{(0)}$, then $U$ is called an open bisection. If $\mathcal{G}$ is étale and $U \subseteq \mathcal{G}$ is open, the restrictions $\left.\boldsymbol{c}\right|_{U}$ and $\left.\boldsymbol{d}\right|_{U}$ are continuous open maps, so they need only be injective for $U$ to be an open bisection. An equivalent definition of an étale groupoid is a topological groupoid that has a base of open bisections. If $\mathcal{G}$ is étale and $\mathcal{G}^{(0)}$ is Hausdorff, then $\mathcal{G}$ is locally Hausdorff, because all the open bisections are homeomorphic to subspaces of $\mathcal{G}^{(0)}$. Another property of étale groupoids is that for any $x \in \mathcal{G}^{(0)}$, the fibres $x \mathcal{G}$ and $\mathcal{G} x$ are discrete spaces. Consequently, a groupoid with only one unit (i.e., a group) is étale if and only if it has the discrete topology.

Definition 1.5. An ample groupoid is a topological groupoid with Hausdorff unit space and a base of compact open bisections.

If $\mathcal{G}$ is an ample groupoid, the notation $B^{\mathrm{co}}(\mathcal{G})$ stands for the set of all nonempty compact open bisections in $\mathcal{G}$, and $\mathcal{B}\left(\mathcal{G}^{(0)}\right)$ stands for the set of nonempty compact open subsets of $\mathcal{G}^{(0)}$.

Recall that a topological space is said to be totally disconnected if the only nonempty connected subsets are singletons, and 0-dimensional if every point has a neighbourhood base of clopen (i.e., closed and open) sets. These two notions are equivalent if the space is locally compact and Hausdorff [70, Theorems 29.5 \& 29.7]. The following proposition is similar to [39, Proposition 4.1]. It is useful for reconciling slightly different definitions in the literature (e.g., [27]) and for checking when an étale groupoid is ample. 
Proposition 1.6. Let $\mathcal{G}$ be an étale groupoid such that $\mathcal{G}^{(0)}$ is Hausdorff. Then the following are equivalent:

(1) $\mathcal{G}$ is an ample groupoid;

(2) $\mathcal{G}^{(0)}$ is locally compact and totally disconnected;

(3) Every open bisection is locally compact and totally disconnected.

Proof. (1) $\Rightarrow(2)$ Let $U \subseteq \mathcal{G}^{(0)}$ be open. Since $\mathcal{G}$ is ample and $\mathcal{G}^{(0)}$ is open, for every $x \in U$ there is a compact open bisection $B$ such that $x \in B \subseteq U \subseteq \mathcal{G}^{(0)}$. Moreover, $\mathcal{G}^{(0)}$ is Hausdorff, so $B$ is closed. This shows that $\mathcal{G}^{(0)}$ is locally compact and 0-dimensional (hence totally disconnected).

$(2) \Rightarrow(3)$ Every open bisection is homeomorphic to an open subspace of $\mathcal{G}^{(0)}$, so it is totally disconnected and locally compact.

$(3) \Rightarrow(1)$ Let $U$ be open in $\mathcal{G}$, and $x \in U$. Since $\mathcal{G}$ is étale, it has a base of open bisections, so there is an open bisection $B$ with $x \in B \subseteq U$. Moreover, $B$ is Hausdorff, locally compact, and totally disconnected, so $x$ has a compact neighbourhood $W \subseteq B$ and a clopen neighbourhood $V \subseteq W$. Since $B$ is Hausdorff and $V$ is closed in $W$, it follows that $V$ is compact. Moreover, $V$ is an open bisection because $B$ is an open bisection. So, $V$ is a compact open bisection. This shows that $\mathcal{G}$ has a base of compact open bisections, so $\mathcal{G}$ is ample.

Remark 1.7. If $\mathcal{G}$ is a topological groupoid and $\mathcal{E}$ is a subgroupoid of $\mathcal{G}$, then $\mathcal{E}$ is automatically a topological groupoid with the topology it inherits from $\mathcal{G}$. If $\mathcal{G}$ is étale, then so is $\mathcal{E}$. However, if $\mathcal{G}$ is ample, then it is not guaranteed that $\mathcal{E}$ is ample. Indeed, by Proposition 1.6 (2), a subgroupoid $\mathcal{E}$ of an ample groupoid $\mathcal{G}$ is ample if and only if $\mathcal{E}^{(0)}$ is locally compact. In particular, $\mathcal{E}$ is ample if $\mathcal{G}$ is ample and $\mathcal{E}^{(0)}$ is either open or closed in $\mathcal{G}^{(0)}$.

The following lemma is similar to [56, Proposition 2.2.4], but with slightly different assumptions.

Lemma 1.8. Let $\mathcal{G}$ be an étale groupoid where $\mathcal{G}^{(0)}$ is Hausdorff. If $A, B, C \subseteq \mathcal{G}$ are compact open bisections, then

(1) $A^{-1}=\left\{a^{-1} \mid a \in A\right\}$ and $A B=\left\{a b \mid(a, b) \in(A \times B) \cap \mathcal{G}^{(2)}\right\}$ are compact open bisections.

(2) If $\mathcal{G}$ is Hausdorff, then $A \cap B$ is a compact open bisection.

Proof. (1) Firstly, $A^{-1}=\boldsymbol{i}(A)$ is compact and open because $\boldsymbol{i}$ is a homeomorphism. Clearly, $A^{-1}$ is an open bisection. Secondly, note that $A B$ might be empty, in which case it is trivially a compact open bisection. Otherwise, $(A \times B) \cap \mathcal{G}^{(2)}$ is compact because $\mathcal{G}^{(2)}$ is closed in $\mathcal{G} \times \mathcal{G}$, and $A B=\boldsymbol{m}\left((A \times B) \cap \mathcal{G}^{(2)}\right)$ is compact because $\boldsymbol{m}$ is continuous. Since $\boldsymbol{m}$ is a local homeomorphism, it is an open map, and $A B=\boldsymbol{m}\left((A \times B) \cap \mathcal{G}^{(2)}\right)$ is open. To prove that it is a bisection, suppose $(a, b)$ is a composable pair in $A \times B$ and $\boldsymbol{d}(a b)=x$. Since $A$ and $B$ are bisections, $b$ is the unique element in $B$ having $\boldsymbol{d}(b)=x$, and $a$ is the unique element of $A$ having $\boldsymbol{d}(a)=\boldsymbol{c}(b)$. So, $\left.\boldsymbol{d}\right|_{A B}$ is injective. Similarly, $\left.\boldsymbol{c}\right|_{A B}$ is injective.

(2) It is trivial that $A \cap B$ is an open bisection. The Hausdorff property on $\mathcal{G}$ implies $A$ and $B$ are closed, so $A \cap B$ is closed, hence compact.

Lemma 1.8 remains true if the words "compact" or "open", or both, are removed throughout the statement. Using Lemma 1.8 (2) with mathematical induction shows that when an ample groupoid is Hausdorff, its set of compact open bisections is closed under finite intersections. The converse to this statement is also true: an ample groupoid is Hausdorff if the set of compact open bisections is closed under finite intersections (see [63, Proposition 3.7]).

The main takeaway from Lemma 1.8 (1) is that the compact open bisections in an ample groupoid are important for two reasons: they generate the topology, and they can be multiplied and inverted in a way that is consistent with an algebraic structure called an inverse semigroup. An inverse semigroup 
is a semigroup $S$ such that every $s \in S$ has a unique inverse $s^{*} \in S$ with the property $s s^{*} s=s$ and $s^{*} s s^{*}=s^{*}$

Example 1.9. If $X$ is a set, a partial symmetry of $X$ is a bijection $s: \operatorname{dom}(s) \rightarrow \operatorname{cod}(s)$ where $\operatorname{dom}(s)$ and $\operatorname{cod}(s)$ are (possibly empty) subsets of $X$. Two partial symmetries $s$ and $t$ are composed in the way that binary relations are composed, so that $s t: \operatorname{dom}(s t) \rightarrow \operatorname{cod}(s t)$ is the map $s t(x)=s(t(x))$ for all $x \in X$ such that $s(t(x))$ makes sense. It is not necessary to have $\operatorname{dom}(s)=\operatorname{cod}(t)$ in order to compose $s$ and $t$. The semigroup $\mathcal{I}_{X}$ of partial symmetries on $X$ is called the symmetric inverse semigroup on $X$. The Wagner-Preston Theorem is an analogue of Cayley's Theorem for groups: every inverse semigroup $S$ has an embedding into $\mathcal{I}_{S}$.

The following result is an adaptation of [56, Proposition 2.2.3].

Proposition 1.10. If $\mathcal{G}$ is an ample groupoid, $B^{\mathrm{co}}(\mathcal{G})$ is an inverse semigroup with the inversion and composition rules displayed in Lemma 1.8 (1).

Proof. Lemma $1.8(1)$ proves that $B^{\mathrm{co}}(\mathcal{G})$ is a semigroup and that $A \in B^{\mathrm{co}}(\mathcal{G})$ implies $A^{-1} \in B^{\mathrm{co}}(\mathcal{G})$. If $A \in B^{\mathrm{co}}(\mathcal{G})$ then $A A^{-1}=\boldsymbol{c}(A)$ because all composable pairs in $A \times A^{-1}$ are of the form $\left(a, a^{-1}\right)$ for some $a \in A$. Therefore $A A^{-1} A=\boldsymbol{c}(A) A=A$ and $A^{-1} A A^{-1}=A^{-1} \boldsymbol{c}(A)=A^{-1} \boldsymbol{d}\left(A^{-1}\right)=A^{-1}$. To show that the inverses are unique, suppose $B \in B^{\mathrm{co}}(\mathcal{G})$ satisfies $A B A=A$ and $B A B=B$. Then for all $a \in A$ there exists $b \in B$ such that $a b a=a$. But then $b=a^{-1} a a^{-1}=a^{-1}$. This shows $A^{-1} \subseteq B$. Similarly, $B A B=B$ implies $B^{-1} \subseteq A$ and consequently $B \subseteq A^{-1}$. Therefore $B=A^{-1}$.

The proposition above has shown how to associate an inverse semigroup to an ample groupoid. The connections between ample groupoids and inverse semigroups run much deeper than this. There are at least two ways to associate an ample groupoid $\mathcal{G}$ to an inverse semigroup $S$. The first is the underlying groupoid $\mathcal{G}_{S}$, where the underlying set is $S$, the topology is discrete, the unit space is the set of idempotents in $S$, and $\boldsymbol{d}(s)=s^{*} s$ while $\boldsymbol{c}(s)=s s^{*}$, for every $s \in S$. Composition in $\mathcal{G}_{S}$ is the binary operation from $S$, just restricted to composable pairs. The second way to associate an ample groupoid to an inverse semigroup $S$ is more complicated. It is called the universal groupoid of $S$, and it only differs from the underlying groupoid when $S$ is large (i.e., fails to have some finiteness conditions). The universal groupoid has a topology that makes it ample but not necessarily Hausdorff. The universal groupoid of $S$ is quite powerful (as shown in [63]) because its Steinberg algebra $A_{R}(\mathcal{G}(S))$ is isomorphic to the inverse semigroup algebra $R S$. This takes us beyond our scope and, after all, we still need to define Steinberg algebras.

1.3. Introducing Steinberg algebras. The purpose of this section is to define and characterise the Steinberg algebra of an ample groupoid over a unital commutative ring $R$. Throughout this section, assume $\mathcal{G}$ is an ample groupoid. In order to make sense of continuity for $R$-valued functions, assume $R$ has the discrete topology. The support of a function $f: X \rightarrow R$ is defined as the set $\operatorname{supp} f=\{x \in X \mid$ $f(x) \neq 0\}$. When $X$ has a topology, we say that $f$ is compactly supported if $\operatorname{supp} f$ is compact. If every point $x \in X$ has an open neighbourhood $N$ such that $\left.f\right|_{N}$ is constant, then $f$ is called locally constant. It is easy to prove that $f: X \rightarrow R$ is locally constant if and only if it is continuous. We use the following notation for the characteristic function of a subset $U$ of $\mathcal{G}$ :

$$
\mathbf{1}_{U}: \mathcal{G} \rightarrow R ; \quad \mathbf{1}_{U}(g)= \begin{cases}1 & \text { if } g \in U \\ 0 & \text { if } g \notin U\end{cases}
$$

Let $R^{\mathcal{G}}$ be the set of all functions $f: \mathcal{G} \rightarrow R$. Canonically, $R^{\mathcal{G}}$ has the structure of an $R$-module with operations defined pointwise.

Definition 1.11 (The Steinberg algebra). Let $A_{R}(\mathcal{G})$ be the $R$-submodule of $R^{\mathcal{G}}$ generated by the set:

$\left\{\mathbf{1}_{U} \mid U\right.$ is a Hausdorff compact open subset of $\left.\mathcal{G}\right\}$. 
The convolution of $f, g \in A_{R}(\mathcal{G})$ is defined as

$$
f * g(x)=\sum_{\substack{y \in \mathcal{G} \\ \boldsymbol{d}(y)=\boldsymbol{d}(x)}} f\left(x y^{-1}\right) g(y)=\sum_{\substack{(z, y) \in \mathcal{G}^{(2)} \\ z y=x}} f(z) g(y) \quad \text { for all } x \in \mathcal{G} .
$$

The $R$-module $A_{R}(\mathcal{G})$, with the convolution, is called the Steinberg algebra of $\mathcal{G}$ over $R$.

Example 1.12. If $\Gamma$ is a discrete group, then $A_{R}(\Gamma)$ is isomorphic to $R \Gamma$, the usual group algebra of $\Gamma$ with coefficients in $R$.

We have yet to justify the definition of the convolution in (1.1). The two sums in the formula are equal, by substituting $z=x y^{-1}$. But it should not be taken for granted that the sum is finite, that $*$ is associative, or even that $A_{R}(\mathcal{G})$ is closed under $*$. These facts will be proved later. First, we prove the following result (inspired by [63]) that leads to some alternative descriptions of $A_{R}(\mathcal{G})$ as an $R$-module.

Proposition 1.13. Let $\mathcal{B}$ be a base for $\mathcal{G}$ consisting of Hausdorff compact open sets, with the property:

$$
\left\{\bigcap_{i=1}^{n} B_{i} \mid B_{i} \in \mathcal{B}, \bigcup_{i=1}^{n} B_{i} \text { is Hausdorff }\right\} \subseteq \mathcal{B} \cup\{\emptyset\} .
$$

Then $A_{R}(\mathcal{G})=\operatorname{span}_{R}\left\{\mathbf{1}_{B} \mid B \in \mathcal{B}\right\}$.

Proof. Let $A=\operatorname{span}_{R}\left\{\mathbf{1}_{B} \mid B \in \mathcal{B}\right\}$. From the definition of $A_{R}(\mathcal{G})$, we have $A \subseteq A_{R}(\mathcal{G})$. To prove the other containment, suppose $U$ is a Hausdorff compact open subset of $\mathcal{G}$. It is sufficient to prove that $\mathbf{1}_{U}$ is an $R$-linear combination of finitely many $\mathbf{1}_{B_{i}}$, where each $B_{i} \in \mathcal{B}$. Since $\mathcal{B}$ is a base for the topology on $\mathcal{G}$, we can write $U$ as a union of sets in $\mathcal{B}$, and use the compactness of $U$ to reduce it to a finite union $U=B_{1} \cup \cdots \cup B_{n}$, where $B_{1}, \ldots, B_{n} \in \mathcal{B}$. By the principle of inclusion-exclusion:

$$
\mathbf{1}_{U}=\sum_{k=1}^{n}(-1)^{k-1} \sum_{\substack{I \subseteq\{1, \ldots, n\} \\|I|=k}} \mathbf{1}_{\cap_{i \in I} B_{i}} .
$$

The main assumption ensures that the sets $\cap_{i \in I} B_{i}$ on the right hand side are either empty or in $\mathcal{B}$. Therefore $A_{R}(\mathcal{G}) \subseteq A$.

Corollary 1.14. If $\mathcal{G}$ is Hausdorff and $\mathcal{B}$ is a base of compact open sets that is closed under finite intersections, then $A_{R}(\mathcal{G})=\operatorname{span}_{R}\left\{\mathbf{1}_{B} \mid B \in \mathcal{B}\right\}$.

We remarked after Lemma 1.8 that if $\mathcal{G}$ is non-Hausdorff, $B^{\mathrm{co}}(\mathcal{G})$ is not closed under finite intersections. Strange things can happen in non-Hausdorff spaces and the problem lies in the fact that compact sets are not always closed, and the intersection of two compact sets is not always compact. However, $B^{\mathrm{co}}(\mathcal{G})$ does satisfy the hypothesis of Proposition 1.13.

Corollary 1.15. [63, Proposition 4.3] The Steinberg algebra is generated as an $R$-module by characteristic functions of compact open bisections. That is, $A_{R}(\mathcal{G})=\operatorname{span}_{R}\left\{\mathbf{1}_{B} \mid B \in B^{\mathrm{co}}(\mathcal{G})\right\}$.

Proof. If $B_{1}, \ldots, B_{n} \in B^{\mathrm{co}}(\mathcal{G})$, and $U=\cup_{i} B_{i}$ is Hausdorff, then each $B_{i}$ is closed in $U$ because $U$ is compact, so $\cap_{i} B_{i}$ is closed in $U$. And, $B_{1}$ is a compact set containing the closed set $\cap_{i} B_{i}$, so $\cap_{i} B_{i}$ is compact. Clearly $\cap_{i} B_{i}$ is an open bisection, so $\cap_{i} B_{i} \in B^{\mathrm{co}}(\mathcal{G})$.

Remark 1.16. If $\mathcal{G}$ is an ample groupoid and $\mathcal{E}$ is an open subgroupoid, then $\mathcal{E}$ is also ample (see Remark 1.7). Let $\iota: \mathcal{E} \hookrightarrow \mathcal{G}$ be the inclusion homomorphism. There is a canonical monomorphism $m: A_{R}(\mathcal{E}) \hookrightarrow A_{R}(\mathcal{G})$, linearly extended from $\mathbf{1}_{U} \mapsto \mathbf{1}_{\iota(U)}$ for every Hausdorff compact open set $U \subseteq \mathcal{E}$. If $\mathcal{E}$ is closed, $m$ has a left inverse $e: A_{R}(\mathcal{G}) \rightarrow A_{R}(\mathcal{E})$, linearly extended from $\mathbf{1}_{U} \mapsto \mathbf{1}_{U \cap \mathcal{E}}$ for every Hausdorff compact open set $U \subseteq \mathcal{G}$.

We still owe a proof that the convolution, from equation (1.1), is well-defined and gives an $R$-algebra structure to $A_{R}(\mathcal{G})$. The next two results are similar to [63, Propositions $\left.4.5 \& 4.6\right]$. 
Lemma 1.17. Let $A, B, C \in B^{\mathrm{co}}(\mathcal{G})$ and $r, s \in R$. Then:

(1) $\mathbf{1}_{A^{-1}}(x)=\mathbf{1}_{A}\left(x^{-1}\right)$ for all $x \in \mathcal{G}$;

(2) $\mathbf{1}_{A} * \mathbf{1}_{B}=\mathbf{1}_{A B}$;

Proof. (1) We have $x \in A^{-1}$ if and only if $x^{-1} \in A$.

(2) Let $x \in \mathcal{G}$. By definition:

$$
\mathbf{1}_{A} * \mathbf{1}_{B}(x)=\sum_{\substack{y \in \mathcal{G} \\ d(y)=\boldsymbol{d}(x)}} \mathbf{1}_{A}\left(x y^{-1}\right) \mathbf{1}_{B}(y)=\sum_{\substack{y \in B \\ d(y)=\boldsymbol{d}(x)}} \mathbf{1}_{A}\left(x y^{-1}\right)
$$

Assume $x$ is of the form $x=a b$ where $a \in A$ and $b \in B$. Since $B$ is a bisection, $b$ is the only element of $B$ having $\boldsymbol{d}(b)=\boldsymbol{d}(x)$, and it follows that

$$
\mathbf{1}_{A} * \mathbf{1}_{B}(x)=\mathbf{1}_{A}\left(x b^{-1}\right)=\mathbf{1}_{A}(a)=1 .
$$

On the other hand, assume $x \notin A B$. If there is $y \in B$ such that $\boldsymbol{d}(y)=\boldsymbol{d}(x)$, then $x y^{-1} \notin A$, for if it were, then $x y^{-1} y=x$ would be in $A B$. Therefore (1.2) yields $\mathbf{1}_{A} * \mathbf{1}_{B}(x)=0$.

Lemma 1.17 (2) implies that characteristic functions of compact open subsets of the unit space can be multiplied pointwise. That is, if $V, W \in \mathcal{B}\left(\mathcal{G}^{(0)}\right)$ then $V W=V \cap W=W V$ and $\mathbf{1}_{V} * \mathbf{1}_{W}(x)=\mathbf{1}_{V}(x) \mathbf{1}_{W}(x)$ for all $x \in \mathcal{G}$. As $\mathcal{G}^{(0)}$ is open in any ample groupoid $\mathcal{G}$, by Remark 1.16, there is a commutative subalgebra $A_{R}\left(\mathcal{G}^{(0)}\right) \hookrightarrow A_{R}(\mathcal{G})$.

The ingredients of an $R$-algebra are an $R$-module $A$ and a binary operation $A \times A \rightarrow A$. The binary operation should be $R$-linear in the first and second arguments (that is, bilinear), and it should be associative. There does not need to be a multiplicative identity. It is tedious to prove that $*$ is associative from its definition in (1.1), so a proof was omitted in [63].

Proposition 1.18. The $R$-module $A_{R}(\mathcal{G})$, equipped with the convolution, is an $R$-algebra.

Proof. We need to show that the image of $*: A_{R}(\mathcal{G}) \times A_{R}(\mathcal{G}) \rightarrow R^{\mathcal{G}}$ is contained in $A_{R}(\mathcal{G})$, and that $*$ is associative and bilinear. Bilinearity can be proved quite easily from formula (1.1). Recall from Corollary 1.15 that the elements of $A_{R}(\mathcal{G})$ are $R$-linear combinations of characteristic functions of compact open bisections. If $f=\sum_{i} a_{i} \mathbf{1}_{A_{i}}, g=\sum_{j} b_{j} \mathbf{1}_{B_{j}}$, and $h=\sum_{k} c_{k} \mathbf{1}_{C_{k}}$, where the sums are finite, and $A_{i}, B_{j}, C_{k} \in B^{\mathrm{co}}(\mathcal{G})$ while $a_{i}, b_{j}, c_{k} \in R$ for all $i, j, k$, then

$$
(f * g) * h=\sum_{i} \sum_{j} \sum_{k} a_{i} b_{j} c_{k} \mathbf{1}_{\left(A_{i} B_{j}\right) C_{k}}=\sum_{i} \sum_{j} \sum_{k} a_{i} b_{j} c_{k} \mathbf{1}_{A_{i}\left(B_{j} C_{k}\right)}=f *(g * h),
$$

using Lemma $1.17(2)$ and the bilinearity of $*$. This proves $*$ is associative. Evidently, $f * g=$ $\sum_{i, j} a_{i} b_{j} \mathbf{1}_{A_{i} B_{j}} \in A_{R}(\mathcal{G})$, so $A_{R}(\mathcal{G})$ is closed under $*$.

It is often useful to think of $*$ simply as the extension of the rule $\mathbf{1}_{A} * \mathbf{1}_{B}=\mathbf{1}_{A B}$ for all pairs $A, B \in B^{\mathrm{co}}(\mathcal{G})$, rather than the more complicated-looking expression (1.1) that we first defined it with. Moreover, one can infer from it that $A_{R}(\mathcal{G})$ is a homomorphic image of the semigroup algebra of $B^{\mathrm{co}}(\mathcal{G})$ with coefficients in $R$.

Proposition 1.19. If $\mathcal{G}$ is Hausdorff and ample, then

$$
A_{R}(\mathcal{G})=\{f: \mathcal{G} \rightarrow R \mid f \text { is locally constant, compactly supported }\} .
$$

Moreover, if $\mathcal{B}$ is a base for $\mathcal{G}$ consisting of compact open sets, such that $\mathcal{B}$ is closed under finite intersections and relative complements, then every nonzero $f \in A_{R}(\mathcal{G})$ is of the form $f=\sum_{i=1}^{m} r_{i} \mathbf{1}_{B_{i}}$, where $r_{1}, \ldots, r_{n} \in R \backslash\{0\}$ and $B_{1}, \ldots, B_{n} \in \mathcal{B}$ are mutually disjoint. 
Proof. Let $A$ be the set of locally constant, compactly supported $R$-valued functions on $\mathcal{G}$. Let $\mathcal{B}$ be a base of compact open sets for $\mathcal{G}$, such that $\mathcal{B}$ is closed under finite intersections and relative complements. (A worthy candidate for $\mathcal{B}$ is $B^{\mathrm{co}}(\mathcal{G})$.) If $0 \neq f \in A_{R}(\mathcal{G})$ then according to Corollary $1.14, f=\sum_{i=1}^{n} s_{i} \mathbf{1}_{D_{i}}$ for some basic open sets $D_{i} \in \mathcal{B}$ and nonzero scalars $s_{i} \in R$. We aim to rewrite it as a linear combination of characteristic functions of disjoint open sets. If $s \in \operatorname{im} f \backslash\{0\}$, then we have the expression:

$$
f^{-1}(s)=\bigcup_{\substack{I \subseteq\{1, \ldots, n\} \\ s=\sum_{i \in I} s_{i}}} B_{I}, \quad \text { where } \quad B_{I}=\bigcap_{\substack{i \in I \\ j \notin I}} D_{i} \backslash D_{j}
$$

By assumption, each nonempty $B_{I}$ in the expression is an element of $\mathcal{B}$; in particular, each $B_{I}$ is compact and open. Finite unions preserve openness and compactness, so $f^{-1}(s)$ is open and compact for every nonzero $s \in \operatorname{im} f$. It follows that $f^{-1}(0)=\mathcal{G} \backslash\left(\bigcup_{s \in \operatorname{im} f \backslash\{0\}} f^{-1}(s)\right)$ is open. Therefore $f$ is locally constant. As $f$ is a linear combination of $n$ characteristic functions, it is clear that $|\operatorname{im} f \backslash\{0\}| \leq$ $2^{n}$. Being a finite union of compact sets, supp $f=\bigcup_{s \in \operatorname{im} f \backslash\{0\}} f^{-1}(s)$ is compact. Thus $f \in A$, and this shows $A_{R}(\mathcal{G}) \subseteq A$. To prove the other containment, that $A \subseteq A_{R}(\mathcal{G})$, suppose $f \in A$. As $f$ is continuous and supp $f$ is compact, $f(\operatorname{supp} f)=\operatorname{im} f \backslash\{0\}$ is compact in $R$, so it must be finite. Let $\operatorname{im} f \backslash\{0\}=\left\{r_{1}, \ldots, r_{n}\right\}$. Then each set $U_{i}=f^{-1}\left(r_{i}\right)$ is clopen because $f$ is continuous, and compact because $U_{i} \subseteq \operatorname{supp} f$. Hence $f=\sum_{i=1}^{n} r_{i} \mathbf{1}_{U_{i}} \in A_{R}(\mathcal{G})$, and this shows $A \subseteq A_{R}(\mathcal{G})$.

To prove the "moreover" part, we look again at (1.4). If $I, J \subseteq\{1, \ldots, n\}$ and $I \neq J$ then $B_{I} \cap B_{J}=\emptyset$. Therefore, $f \in A_{R}(\mathcal{G})$ can be written as an $R$-linear combination of characteristic functions of disjoint basic open sets in $\mathcal{B}$ :

$$
f=\sum_{s \in \operatorname{im} f \backslash\{0\}} s \mathbf{1}_{f^{-1}(s)}=\sum_{s \in \operatorname{im} f \backslash\{0\}} \sum_{\substack{I \subseteq\{1, \ldots, n\} \\ s=\sum_{i \in I} s_{i}}} s \mathbf{1}_{B_{I}} .
$$

1.4. Properties of Steinberg algebras. It is useful to know when $A_{R}(\mathcal{G})$ is unital or has some property that is nearly as good. The answer is quite easy, and we show it below. We use the definition that a ring (or $R$-algebra) $A$ is locally unital if there is a set of commuting idempotents $E \subseteq A$, called local units, with the property: for every finite subset $\left\{a_{1}, \ldots, a_{n}\right\} \subseteq A$, there is a local unit $e \in E$ with $e a_{i}=a_{i}=a_{i} e$ for every $1 \leq i \leq n$. Equivalently, $A$ is the direct limit of unital subrings: $A=\underset{\longrightarrow}{\longrightarrow} \lim _{e \in E} e A e$. The directed system is facilitated by the partial order, $e \leq e^{\prime}$ if $e e^{\prime}=e=e^{\prime} e$, and the connecting homomorphisms (which need not be unit-preserving) are the inclusions $e A e \hookrightarrow e^{\prime} A e^{\prime}$ for $e \leq e^{\prime}$.

In many respects, working with locally unital rings is like working with unital rings. Every locally unital ring $A$ is idempotent (i.e., $A^{2}=A$ ) and if $I \subseteq A$ is an ideal, then $A I=I=I A$. If $A$ is an $R$-algebra with local units, then the ring ideals of $A$ are always $R$-algebra ideals (which, by definition, should be $R$-submodules of $A$ ). These facts are not true in general for arbitrary non-unital rings. Locally unital rings and algebras are always homologically unital, in the sense of [53, Definition 1.4.6], which essentially means that they have well-behaved homology. The classical Morita Theorems, with slight adjustments, are valid for rings with local units (see [10]).

Proposition 1.20. [63, Proposition 4.11] , [25, Lemma 2.6]. Let $\mathcal{G}$ be an ample groupoid. Then $A_{R}(\mathcal{G})$ is locally unital. Moreover, $A_{R}(\mathcal{G})$ is unital if and only if $\mathcal{G}^{(0)}$ is compact.

Proof. We prove the "moreover" part first. If $\mathcal{G}^{(0)}$ is compact, then it is a compact open bisection, and $\mathbf{1}_{\mathcal{G}^{(0)}} \in A_{R}(\mathcal{G})$. Following Lemma $1.17(2), \mathbf{1}_{\mathcal{G}^{(0)}} * \mathbf{1}_{B}=\mathbf{1}_{\mathcal{G}^{(0)} B}=\mathbf{1}_{B}=\mathbf{1}_{B \mathcal{G}^{(0)}}=\mathbf{1}_{B} * \mathbf{1}_{\mathcal{G}^{(0)}}$, for every $B \in B^{\mathrm{co}}(\mathcal{G})$. Since $\left\{\mathbf{1}_{B} \mid B \in B^{\mathrm{co}}(\mathcal{G})\right\}$ spans $A_{R}(\mathcal{G})$, it follows by linearity that $\mathbf{1}_{\mathcal{G}(0)} * f=f=f * \mathbf{1}_{\mathcal{G}(0)}$ for every $f \in A_{R}(\mathcal{G})$. This proves that $\mathbf{1}_{\mathcal{G}^{(0)}}$ is the multiplicative identity in $A_{R}(\mathcal{G})$.

Conversely, suppose $A_{R}(\mathcal{G})$ has a multiplicative identity called $\xi$. The first step is to show that $\xi=\mathbf{1}_{\mathcal{G}^{(0)}}$. Let $x \in \mathcal{G}$ and let $V \subseteq \mathcal{G}^{(0)}$ be a compact open set containing $\boldsymbol{d}(x)$. Then $V$ must be Hausdorff because 
$\mathcal{G}^{(0)}$ is, so $\mathbf{1}_{V} \in A_{R}(\mathcal{G})$. If $x \notin \mathcal{G}^{(0)}$, then

$$
0=\mathbf{1}_{V}(x)=\xi * \mathbf{1}_{V}(x)=\sum_{y \in \mathcal{G} \boldsymbol{d}(x)} \xi\left(x y^{-1}\right) \mathbf{1}_{V}(y)=\sum_{y \in V \cap \mathcal{G} \boldsymbol{d}(x)} \xi\left(x y^{-1}\right)=\xi(x)
$$

because $V \cap \mathcal{G} \boldsymbol{d}(x)=\{\boldsymbol{d}(x)\}$. Similarly, if $x \in \mathcal{G}^{(0)}$ then $x=\boldsymbol{d}(x) \in V$ and

$$
1=\mathbf{1}_{V}(x)=\xi * \mathbf{1}_{V}(x)=\xi(x) .
$$

This shows that $\xi=\mathbf{1}_{\mathcal{G}^{(0)}}$. The second step is to show that $\mathbf{1}_{\mathcal{G}^{(0)}} \in A_{R}(\mathcal{G})$ implies $\mathcal{G}^{(0)}$ is compact. By the definition of $A_{R}(\mathcal{G})$, there exist scalars $r_{1}, \ldots, r_{n} \in R \backslash\{0\}$ and compact open sets $U_{1}, \ldots, U_{n} \subseteq \mathcal{G}$ such that $\mathbf{1}_{\mathcal{G}^{(0)}}=r_{1} \mathbf{1}_{U_{1}}+\cdots+r_{n} \mathbf{1}_{U_{n}}$. Then $\mathcal{G}^{(0)} \subseteq U_{1} \cup \cdots \cup U_{n}$ and consequently $\mathcal{G}^{(0)}=\boldsymbol{d}\left(U_{1}\right) \cup \cdots \cup \boldsymbol{d}\left(U_{n}\right)$. Each of the sets $\boldsymbol{d}\left(U_{1}\right), \ldots, \boldsymbol{d}\left(U_{n}\right)$ is compact (because $\boldsymbol{d}$ is continuous), so $\mathcal{G}^{(0)}$ is compact.

To show that $A_{R}(\mathcal{G})$ is locally unital for all ample groupoids $\mathcal{G}$, suppose $F=\left\{f_{1}, \ldots, f_{m}\right\}$ is a finite subset of $A_{R}(\mathcal{G})$. Since $A_{R}(\mathcal{G})$ is spanned by $\left\{\mathbf{1}_{B} \mid B \in B^{\mathrm{co}}(\mathcal{G})\right\}$, there exist finite subsets $\left\{B_{1}, \ldots, B_{n}\right\} \subseteq B^{\mathrm{co}}(\mathcal{G})$ and $\left\{r_{i, j} \mid 1 \leq i \leq n, 1 \leq j \leq m\right\} \subseteq R$ such that $f_{j}=r_{1, j} \mathbf{1}_{B_{1}}+\cdots+r_{n, j} \mathbf{1}_{B_{n}}$ for all $1 \leq j \leq m$. Let $X=\boldsymbol{d}\left(B_{1}\right) \cup \cdots \cup \boldsymbol{d}\left(B_{n}\right) \cup \boldsymbol{c}\left(B_{1}\right) \cup \cdots \cup \boldsymbol{c}\left(B_{n}\right)$. Then $X \subseteq G^{(0)}$ is compact and open because it is a finite union of compact open sets, and $X$ is Hausdorff because it is a subset of $\mathcal{G}^{(0)}$, so $\mathbf{1}_{X} \in A_{R}(\mathcal{G})$. Clearly, $X B_{i}=B_{i}=B_{i} X$, so $\mathbf{1}_{X} * \mathbf{1}_{B_{i}}=\mathbf{1}_{B_{i}}=\mathbf{1}_{B_{i}} * \mathbf{1}_{X}$, for all $1 \leq i \leq n$. By linearity, $\mathbf{1}_{X} * f_{j}=f_{j}=f_{j} * \mathbf{1}_{X}$ for all $1 \leq j \leq m$. The conclusion is that $E=\left\{\mathbf{1}_{X} \mid X \in \mathcal{B}\left(\mathcal{G}^{(0)}\right)\right\}$ is a set of local units for $A_{R}(\mathcal{G})$.

The characteristic of a ring $A$, written char $A$, is defined as the least positive integer $n$ such that $n \cdot a=0$ for all $a \in A$, or 0 if no such $n$ exists. If $A$ has a set of local units $E$, the characteristic of $A$ can be defined as the least $n$ such that $n \cdot e=0$ for all $e \in E$, or 0 if no such $n$ exists.

Proposition 1.21. For any ample groupoid $\mathcal{G}$, $\operatorname{char} A_{R}(\mathcal{G})=\operatorname{char} R$.

Proof. If $n$ is a positive integer, $n \cdot \mathbf{1}_{U}=0$ for all $U \in \mathcal{B}\left(\mathcal{G}^{(0)}\right)$ if and only if $n \cdot 1=0$.

Given a topological groupoid $\left(\mathcal{G}, \mathcal{G}^{(0)}, \boldsymbol{d}, \boldsymbol{c}, \boldsymbol{m}, \boldsymbol{i}\right)$, the opposite groupoid is:

$$
\mathcal{G}^{\mathrm{op}}=\left(\mathcal{G}, \mathcal{G}^{(0)}, \boldsymbol{d}^{\mathrm{op}}, \boldsymbol{c}^{\mathrm{op}}, \boldsymbol{m}^{\mathrm{op}}, \boldsymbol{i}\right)
$$

where $\boldsymbol{d}^{\mathrm{op}}=\boldsymbol{c}, \boldsymbol{c}^{\mathrm{op}}=\boldsymbol{d}$, and $\boldsymbol{m}^{\mathrm{op}}(x, y)=\boldsymbol{m}(y, x)$ for any $x, y$ with $\boldsymbol{c}(x)=\boldsymbol{d}(y)$. We call the opposite groupoid $\mathcal{G}^{\text {op }}$ to distinguish it from $\mathcal{G}$, even though they have the same underlying sets. We assume $\mathcal{G}^{\text {op }}$ has the same topology as $\mathcal{G}$. Naturally, the inversion map $i: \mathcal{G} \rightarrow \mathcal{G}^{\text {op }}$ is an isomorphism of topological groupoids.

If $A$ is a ring, an involution on $A$ is an additive, anti-multiplicative map $\tau: A \rightarrow A$ such that $\tau^{2}=\operatorname{id}_{A}$. If $A$ has an involution, it is called an involutive ring or $*$-ring. If $\mathcal{G}$ is an ample groupoid, $f \mapsto f \circ \boldsymbol{i}$ is a canonical involution on $A_{R}(\mathcal{G})$ that makes it a $*$-algebra. More generally, if there is an involution - : $R \rightarrow R$, written as $r \mapsto \bar{r}$, then $f \mapsto \overline{f \circ \boldsymbol{i}}$ is an involution on $A_{R}(\mathcal{G})$. To summarise:

Proposition 1.22. Let $\mathcal{G}$ be an ample groupoid. There are canonical isomorphisms $\mathcal{G} \cong \mathcal{G}^{\mathrm{op}}$ and $A_{R}(\mathcal{G}) \cong$ $A_{R}\left(\mathcal{G}^{\mathrm{op}}\right) \cong A_{R}(\mathcal{G})^{\mathrm{op}}$. Moreover, to each involution ${ }^{-}: R \rightarrow R$ is associated a canonical involution on $A_{R}(\mathcal{G})$, namely $f \mapsto \overline{f \circ \boldsymbol{i}}$ for all $f \in A_{R}(\mathcal{G})$.

This kind of symmetry is very nice to work with. It implies, for example, that the category of left $A_{R}(\mathcal{G})$ modules is isomorphic to the category of right $A_{R}(\mathcal{G})$-modules, and the lattice of left ideals in $A_{R}(\mathcal{G})$ is isomorphic to the lattice of right ideals. Many important notions, like left and right primitivity, are equivalent for involutive algebras (or more generally, self-opposite algebras).

1.5. First examples. One or two of the results in this section will be useful later on, but mostly they are just interesting in their own right. Presumably, most of this content is already known, but we do not adhere closely to any references. 
Given two groupoids $\left(\mathcal{G}_{1}, \boldsymbol{d}_{1}, \boldsymbol{c}_{1}, \boldsymbol{m}_{1}, \boldsymbol{i}_{1}\right)$ and $\left(\mathcal{G}_{2}, \boldsymbol{d}_{2}, \boldsymbol{c}_{2}, \boldsymbol{m}_{2}, \boldsymbol{i}_{2}\right)$, their disjoint union $\mathcal{G}_{1} \sqcup \mathcal{G}_{2}$ has the structure of a groupoid with unit space $\mathcal{G}_{1}^{(0)} \sqcup \mathcal{G}_{2}^{(0)}$, set of composable pairs $\mathcal{G}_{1}^{(2)} \sqcup \mathcal{G}_{2}^{(2)}$, and the following structure maps: for all $x_{1}, y_{1} \in \mathcal{G}_{1}$ and $x_{2}, y_{2} \in \mathcal{G}_{2}$,

$$
\boldsymbol{d}\left(x_{i}\right)=\boldsymbol{d}_{i}\left(x_{i}\right), \quad \boldsymbol{c}\left(x_{i}\right)=\boldsymbol{c}_{i}\left(x_{i}\right), \quad \boldsymbol{i}\left(x_{i}\right)=\boldsymbol{i}_{i}\left(x_{i}\right), \quad \boldsymbol{m}\left(x_{i}, y_{i}\right)=\boldsymbol{m}_{i}\left(x_{i}, y_{i}\right) .
$$

The product $\mathcal{G}_{1} \times \mathcal{G}_{2}$ also has the structure of a groupoid with unit space $\mathcal{G}_{1}^{(0)} \times \mathcal{G}_{2}^{(0)}$, and the following structure maps: for all $x_{1}, y_{1} \in \mathcal{G}_{1}$ and $x_{2}, y_{2} \in \mathcal{G}_{2}$,

$$
\begin{aligned}
\boldsymbol{d}\left(x_{1}, x_{2}\right) & =\left(\boldsymbol{d}_{1}\left(x_{1}\right), \boldsymbol{d}_{2}\left(x_{2}\right)\right), & \boldsymbol{c}\left(x_{1}, x_{2}\right) & =\left(\boldsymbol{c}_{1}\left(x_{1}\right), \boldsymbol{c}_{2}\left(x_{2}\right)\right), \\
\boldsymbol{i}\left(x_{1}, x_{2}\right) & =\left(\boldsymbol{i}_{1}\left(x_{1}\right), \boldsymbol{i}_{2}\left(x_{2}\right)\right), & \boldsymbol{m}\left(\left(x_{1}, x_{2}\right),\left(y_{1}, y_{2}\right)\right) & =\left(\boldsymbol{m}_{1}\left(x_{1}, y_{1}\right), \boldsymbol{m}_{2}\left(x_{2}, y_{2}\right)\right) .
\end{aligned}
$$

These constructions work just as well for the disjoint union or product of arbitrarily many (even infinitely many) groupoids. If $\mathcal{G}_{1}$ and $\mathcal{G}_{2}$ are topological groupoids, then $\mathcal{G}_{1} \sqcup \mathcal{G}_{2}$ (with the coproduct topology) and $\mathcal{G}_{1} \times \mathcal{G}_{2}$ (with the product topology) are again topological groupoids. The properties of being étale or ample are preserved by arbitrary disjoint unions and finite products.

Proposition 1.23. Let $\mathcal{G}_{1}$ and $\mathcal{G}_{2}$ be ample groupoids. The Steinberg algebra of $\mathcal{G}_{1} \sqcup \mathcal{G}_{2}$ is a direct sum of two ideals: $A_{R}\left(\mathcal{G}_{1} \sqcup \mathcal{G}_{2}\right) \cong A_{R}\left(\mathcal{G}_{1}\right) \oplus A_{R}\left(\mathcal{G}_{2}\right)$.

Proof. Let $I_{1}=\left\{f_{1} \in A_{R}\left(\mathcal{G}_{1} \sqcup \mathcal{G}_{2}\right) \mid \operatorname{supp} f_{1} \subseteq \mathcal{G}_{1}\right\}$ and $I_{2}=\left\{f_{2} \in A_{R}\left(\mathcal{G}_{1} \sqcup \mathcal{G}_{2}\right) \mid \operatorname{supp} f_{2} \subseteq \mathcal{G}_{2}\right\}$. Recall from Remark 1.16 that $I_{1} \cong A_{R}\left(\mathcal{G}_{1}\right)$ and $I_{2} \cong A_{R}\left(\mathcal{G}_{2}\right)$. Every $f \in A_{R}\left(\mathcal{G}_{1} \sqcup \mathcal{G}_{2}\right)$ decomposes as $f=f_{1}+f_{2}$ where $f_{i} \in I_{i}$ are defined as:

$$
f_{i}(x)= \begin{cases}f(x) & \text { if } x \in \mathcal{G}_{i} \\ 0 & \text { if } x \notin \mathcal{G}_{i}\end{cases}
$$

for $i=1,2$. We claim $I_{1}$ and $I_{2}$ are orthogonal ideals (that is, $I_{1} * I_{2}=0$ ). For all $f_{1} \in I_{1}, f_{2} \in I_{2}$, and $x \in \mathcal{G}_{1} \sqcup \mathcal{G}_{2}, f_{1} * f_{2}(x)=\sum_{a b=x} f_{1}(a) f_{2}(b)$. So, $\operatorname{supp}\left(f_{1} * f_{2}\right) \subseteq \operatorname{supp}\left(f_{1}\right) \operatorname{supp}\left(f_{2}\right) \subseteq \mathcal{G}_{1} \mathcal{G}_{2}=\emptyset$. This implies $I_{1}$ and $I_{2}$ are ideals, and $A_{R}\left(\mathcal{G}_{1} \sqcup \mathcal{G}_{2}\right)=I_{1} \oplus I_{2} \cong A_{R}\left(\mathcal{G}_{1}\right) \oplus A_{R}\left(\mathcal{G}_{2}\right)$.

By mathematical induction, the Steinberg algebra of a finite disjoint union of ample groupoids is isomorphic to the direct sum of their respective Steinberg algebras.

Like in [2, Notation 2.6.3], we have reasons to consider matrix rings of a slightly more general nature than usual.

Definition 1.24 (Matrix rings). Let $A$ be a ring (not necessarily commutative or unital). If $n$ is a positive integer, we write $M_{n}(A)$ for the ring of $n \times n$ matrices with entries in $A$. If $\Lambda$ is a set (not necessarily finite) we define $M_{\Lambda}(A)$ to be the ring of square matrices, with rows and columns indexed by $\Lambda$, having entries in $A$ and only finitely many nonzero entries.

Note that $M_{\Lambda}(A)$ is the direct limit of the finite-sized matrix rings associated to finite subsets of $\Lambda$. Also, $M_{\Lambda}(A)$ is unital if and only if $A$ is unital and $\Lambda$ is finite. The notation $\left[a_{i j}\right]$ stands for the matrix in $M_{n}(A)$, or $M_{\Lambda}(A)$, with $a_{i j}$ in its $(i, j)$-entry. Let $\mathcal{N}=\{1, \ldots, n\}^{2}$ be the transitive principal groupoid on $n$ elements, with the discrete topology, as seen in Example 1.3 (c).

Proposition 1.25. If $\mathcal{G}$ is a Hausdorff ample groupoid, then $A_{R}(\mathcal{N} \times \mathcal{G}) \cong M_{n}\left(A_{R}(\mathcal{G})\right)$.

Proof. Define the map $F: A_{R}(\mathcal{N} \times \mathcal{G}) \rightarrow M_{n}\left(A_{R}(\mathcal{G})\right)$ :

$$
F(f)=\left[f_{i j}\right], \quad \text { where } f_{i j}(x)=f((i, j), x) \text { for all } f \in A_{R}(\mathcal{N} \times \mathcal{G}),(i, j) \in \mathcal{N} \text {, and } x \in \mathcal{G} \text {. }
$$

If $f \in A_{R}(\mathcal{N} \times \mathcal{G})$, then $f$ is compactly supported and locally constant. The restriction of $f$ to a clopen subset, such as $\{(i, j)\} \times \mathcal{G}$ for some $(i, j) \in \mathcal{N}$, is also compactly supported and locally constant. Therefore $f_{i, j} \in A_{R}(\mathcal{G})$ for all $(i, j) \in \mathcal{N}$. Clearly, $F$ is bijective. Now, let $f, g \in A_{R}(\mathcal{N} \times \mathcal{G})$. For all $(i, j) \in \mathcal{N}$ and $x \in \mathcal{G}$, the convolution formula yields

$$
(f * g)_{i j}(x)=f * g((i, j), x)=\sum_{\substack{(k, \ell, y) \in \mathcal{N} \times \mathcal{G} \\(\ell, \boldsymbol{d}(y))=(j, \boldsymbol{d}(x))}} f\left[((i, j), x)((k, \ell), y)^{-1}\right] g((k, \ell), y)
$$




$$
=\sum_{1 \leq k \leq N} \sum_{\substack{y \in \mathcal{G} \\ d(y)=\boldsymbol{d}(x)}} f\left((i, k), x y^{-1}\right) g((k, j), y)=\sum_{1 \leq k \leq n} f_{i k} * g_{k j}(x)
$$

This shows $F(f * g)=F(f) F(g)$, so $F$ is an isomorphism.

Remark 1.26. As a specialisation of Proposition 1.25 , we obtain $A_{R}(\mathcal{N}) \cong M_{n}(R)$. It is well-known that when $A$ is an $R$-algebra, $M_{n}(A) \cong M_{n}(R) \otimes_{R} A$ (see [18, Example 4.22]). It is also well-known (see [18, Example 4.20]) that if $G$ and $H$ are groups, then $R(G \times H) \cong R G \otimes_{R} R H$. One can show using the standard techniques that when $\mathcal{G}_{1}$ and $\mathcal{G}_{2}$ are arbitrary ample groupoids, there is a surjective homomorphism $A_{R}\left(\mathcal{G}_{1}\right) \otimes_{R} A_{R}\left(\mathcal{G}_{2}\right) \rightarrow A_{R}\left(\mathcal{G}_{1} \times \mathcal{G}_{2}\right)$. An interesting question is: under what circumstances is it an isomorphism?

Suppose $\mathcal{G}$ is a topological groupoid and $\left\{\mathcal{G}_{i}\right\}_{i \in I}$ is a family of open subgroupoids indexed by a directed set $(I, \leq)$, such that $\mathcal{G}=\bigcup_{i \in I} \mathcal{G}_{i}$ and $\mathcal{G}_{i} \subseteq \mathcal{G}_{j}$ whenever $i \leq j$ in $I$. If this happens, we say that $\mathcal{G}$ is the directed union of the subgroupoids $\left\{\mathcal{G}_{i}\right\}_{i \in I}$.

Proposition 1.27. If a Hausdorff ample groupoid $\mathcal{G}$ is the directed union of a family of open subgroupoids $\left\{\mathcal{G}_{i}\right\}_{i \in I}$, then $A_{R}(\mathcal{G})$ is the direct limit of subalgebras $\left\{A_{R}\left(\mathcal{G}_{i}\right)\right\}_{i \in I}$.

Proof. For all $i \leq j$ in $I$, let $\varphi_{i j}: A_{R}\left(\mathcal{G}_{i}\right) \hookrightarrow A_{R}\left(\mathcal{G}_{j}\right)$ and $m_{i}: A_{R}\left(\mathcal{G}_{i}\right) \hookrightarrow A_{R}(\mathcal{G})$ be the canonical embeddings (see Remark 1.16). We claim that for every $f \in A_{R}(\mathcal{G})$, there exists $j \in I$ such that $f \in m_{j}\left(A_{R}\left(\mathcal{G}_{j}\right)\right)$. If $f \in A_{R}(\mathcal{G})$ then $\operatorname{supp} f$ is compact and open. Thus, there is a finite subcover of $\left\{\mathcal{G}_{i}\right\}_{i \in I}$ that covers $\operatorname{supp} f$. If $\operatorname{supp} f \subseteq \mathcal{G}_{i_{1}} \cup \cdots \cup \mathcal{G}_{i_{n}}$, then there exists $j \in I$ with $i_{1}, \ldots, i_{n} \leq j$, using the fact that $(I, \leq)$ is directed. Thus, supp $f \subseteq \mathcal{G}_{j}$, and $\left.f\right|_{\mathcal{G}_{j}}$ is compactly supported and locally constant, whereby $\left.f\right|_{\mathcal{G}_{j}} \in A_{R}\left(\mathcal{G}_{j}\right)$. Finally, this shows $f=m_{j}\left(\left.f\right|_{\mathcal{G}_{j}}\right) \in m_{j}\left(A_{R}\left(\mathcal{G}_{j}\right)\right)$.

Now assume $B$ is an $R$-algebra and $\left\{\beta_{i}\right\}_{i \in I}$ is a family of $R$-homomorphisms $\beta_{i}: A_{R}\left(\mathcal{G}_{i}\right) \rightarrow B$, such that $\beta_{i}=\beta_{j} \varphi_{i j}$ for all $i \leq j$. Then, since every $\varphi_{i j}: A_{R}\left(\mathcal{G}_{i}\right) \rightarrow A_{R}\left(\mathcal{G}_{j}\right)$ is injective, $\beta_{j}$ is an extension of $\beta_{i}$ whenever $i \leq j$. Since $A_{R}(\mathcal{G})=\bigcup_{i \in I} m_{i}\left(A_{R}\left(\mathcal{G}_{i}\right)\right)$, it follows that there is a unique homomorphism $\beta: A_{R}(\mathcal{G}) \rightarrow B$ such that $\beta_{i}=\beta m_{i}$ for all $i \in I$. As such, $A_{R}(\mathcal{G})$ has the universal property for the directed system $\left\{A_{R}\left(\mathcal{G}_{i}\right)\right\}_{i \in I}$, so we can conclude it is the direct limit of that system.

We can now extend Propositions 1.23 and 1.25 to allow infinite index sets. This could have been proved directly, mentioning that the functions in $A_{R}(\mathcal{G})$ have compact supports, but it is nice to demonstrate direct limits.

Proposition 1.28. Let $\mathcal{G}$ be a Hausdorff ample groupoid, and let $\Lambda$ be an infinite set.

(1) If $\mathcal{D}=\Lambda^{2}$ is the transitive principal groupoid on $\Lambda$, equipped with the discrete topology, then $A_{R}(\mathcal{D} \times$ $\mathcal{G}) \cong M_{\Lambda}\left(A_{R}(\mathcal{G})\right)$.

(2) If $\mathcal{G}=\bigsqcup_{\lambda \in \Lambda} \mathcal{G}_{\lambda}$ is the disjoint union of an infinite family of clopen subgroupoids $\left\{\mathcal{G}_{\lambda}\right\}_{\lambda \in \Lambda}$, then $A_{R}(\mathcal{G}) \cong \bigoplus_{\lambda \in \Lambda} A_{R}\left(\mathcal{G}_{\lambda}\right)$.

Proof. (1) Note that $\mathcal{D} \times \mathcal{G}$ is the directed union of the subgroupoids $\mathcal{D}_{F} \times \mathcal{G}$, where $\mathcal{D}_{F}=\left\{\left(d_{1}, d_{2}\right) \in\right.$ $\left.\mathcal{D} \mid d_{1}, d_{2} \in F\right\}$, as $F$ ranges over all the finite subsets of $\Lambda$ ordered by inclusion. By Propositions 1.25 and 1.27, $A_{R}(\mathcal{D} \times \mathcal{G})$ is the direct limit of matrix algebras $A_{R}\left(\mathcal{D}_{F} \times \mathcal{G}\right) \cong M_{F}\left(A_{R}(\mathcal{G})\right)$, and this direct limit is isomorphic to $M_{\Lambda}\left(A_{R}(\mathcal{G})\right)$.

(2) Note that $\mathcal{G}$ is the directed union of the subgroupoids $\mathcal{G}_{F}=\bigsqcup_{\lambda \in F} \mathcal{G}_{\lambda}$, as $F$ ranges over finite subsets of $\Lambda$ ordered by inclusion. By Propositions 1.23 and 1.27, $A_{R}(\mathcal{G})$ is the direct limit of the subalgebras $A_{R}\left(\mathcal{G}_{F}\right) \cong \bigoplus_{\lambda \in F} A_{R}\left(\mathcal{G}_{\lambda}\right)$, and this direct limit is isomorphic to $\bigoplus_{\lambda \in \Lambda} A_{R}\left(\mathcal{G}_{\lambda}\right)$.

Here we describe a class of principal groupoids, called approximately finite groupoids, that was defined by Renault in his influential monograph [59]. 
Example 1.29. Let $X$ be a locally compact, totally disconnected Hausdorff space. Consider it as a groupoid with unit space $X$ and no morphisms outside the unit space. Then $A_{R}(X)$ is the commutative $R$-algebra of locally constant, compactly supported functions $f: X \rightarrow R$, with pointwise addition and multiplication. We adopt the notation $A_{R}(X)=C_{R}(X)$ and drop the $*$ notation for products, because this serves as a reminder that $C_{R}(X)$ is commutative. An ample groupoid is called elementary if it is of the form $\left(\mathcal{N}_{1} \times X_{1}\right) \sqcup \cdots \sqcup\left(\mathcal{N}_{t} \times X_{t}\right)$, where $\mathcal{N}_{1}, \ldots \mathcal{N}_{t}$ are discrete, finite, transitive principal groupoids on $n_{1}, \ldots, n_{t}$ elements, respectively, and $X_{1}, \ldots, X_{n}$ are locally compact, totally disconnected, Hausdorff topological spaces. Using the results of this section:

$$
A_{R}\left(\bigsqcup_{i=1}^{n}\left(\mathcal{N}_{i} \times X_{i}\right)\right) \cong \bigoplus_{i=1}^{t} M_{n_{i}}\left(C_{R}\left(X_{i}\right)\right) .
$$

A groupoid is called approximately finite if it is the directed union of an increasing sequence of elementary groupoids. The Steinberg algebra of an approximately finite groupoid is a direct limit of matricial algebras, each resembling (1.5).

Definition 1.30. A ring $A$ is called von Neumann regular if for every $x \in A$ there exists $y \in A$ such that $x=x y x$.

If $y \in A$ satisfies $x=x y x$ then $y$ is called a von Neumann inverse of $x$. If $R$ is a commutative von Neumann regular ring, then for every $r \in R$ there exists a unique element $s \in R$ such that $r=r^{2} s$ and $s=s^{2} r$ (see [42, Proposition 3.6]).

Proposition 1.31. If $\mathcal{F}$ is an approximately finite groupoid and $R$ is a von Neumann regular unital commutative ring, then $A_{R}(\mathcal{F})$ is von Neumann regular.

Proof. Let $X$ be a locally compact, totally disconnected, Hausdorff topological space, and suppose $R$ is von Neumann regular. To verify that $C_{R}(X)$ is von Neumann regular, take $f \in C_{R}(X)$ and for every $x \in X$ define $g(x)$ to be the unique element of $R$ such that $f(x)=f(x)^{2} g(x)$ and $g(x)=g(x)^{2} f(x)$. Note that $g \in C_{R}(X)$ and $f g f=f$. Now, $C_{R}(X)$ being regular implies $M_{n}\left(C_{R}(X)\right)$ is regular (this could be argued carefully with Morita equivalence, but one finds in [48, Theorem 24] a clever direct proof by induction). A direct sum of regular rings is regular, so any ring of the form (1.5) is regular, provided $R$ is regular. A direct limit of regular rings is regular: each element in the direct limit must belong to a regular subring, and the von Neumann inverse can be chosen from that same subring. Therefore $A_{R}(\mathcal{F})$ is von Neumann regular.

Note that we did not use the assumption that $\mathcal{F}$ is a countable directed union of elementary groupoids; any directed union will do. It is an open problem to characterise von Neumann regularity for Steinberg algebras in groupoid terms; partial progress is achieved in [9].

This next result is a "baby version" of [65, Proposition 3.1], with a new proof. In preparation for it, we briefly remark that every transitive groupoid $\mathcal{G}$ is (algebraically, but not necessarily topologically) isomorphic to the product of a transitive principal groupoid and a group. To construct such an isomorphism, fix a unit $b \in \mathcal{G}^{(0)}$. Let $\Gamma=b \mathcal{G} b$ be the isotropy group based at $b$, and let $\mathcal{P}=\left[\mathcal{G}^{(0)}\right]^{2}$ be the transitive principal groupoid on $\mathcal{G}^{(0)}$. Fix a morphism $h_{y} \in b \mathcal{G} y$ for every $y \in \mathcal{G}^{(0)}$, and define the groupoid isomorphisms:

$$
\begin{aligned}
F: \mathcal{G} \rightarrow \mathcal{P} \times \Gamma, & F(g)=\left((\boldsymbol{c}(g), \boldsymbol{d}(g)), h_{\boldsymbol{c}(g)} g h_{\boldsymbol{d}(g)}^{-1}\right) & \text { for all } g \in \mathcal{G} ; \\
F^{-1}: \mathcal{P} \times \Gamma \rightarrow \mathcal{G}, & F^{-1}((x, y), \gamma)=h_{x}^{-1} \gamma h_{y} & \text { for all } x, y \in \mathcal{G}^{(0)}, \gamma \in \Gamma .
\end{aligned}
$$

Proposition 1.32. Let $\mathbb{K}$ be a field and $\mathcal{G}$ an ample groupoid. Then $A_{\mathbb{K}}(\mathcal{G})$ is finite-dimensional if and only if $\mathcal{G}$ is finite and has the discrete topology. If $\mathcal{O}_{1}, \ldots, \mathcal{O}_{t}$ are the orbits of $\mathcal{G}$, and $\Gamma_{1}, \ldots, \Gamma_{t}$ are the corresponding isotropy groups, then

$$
A_{\mathbb{K}}(\mathcal{G}) \cong \bigoplus_{i=1}^{t} M_{\mathcal{O}_{i}}\left(R \Gamma_{i}\right)
$$


Proof. First of all, if $\mathcal{G}$ is discrete, then $\operatorname{dim}_{\mathbb{K}} A_{\mathbb{K}}(\mathcal{G})=|\mathcal{G}|$, because $\left\{\mathbf{1}_{\{g\}} \mid g \in \mathcal{G}\right\}$ is a basis for $A_{\mathbb{K}}(\mathcal{G})$, by Corollary 1.14. Thus, $A_{\mathbb{K}}(\mathcal{G})$ is finite-dimensional if $\mathcal{G}$ is finite and discrete. Conversely, suppose $A_{\mathbb{K}}(\mathcal{G})$ is finite-dimensional, and let $\left\{f_{1}, \ldots, f_{n}\right\}$ be a basis. The image of each $f_{i}$ is finite, so $\left|\operatorname{im} f_{1} \cup \cdots \cup \operatorname{im} f_{n}\right|$ is bounded by some $M<\infty$. If $\left|\mathcal{G}^{(0)}\right|>M^{n}$ then, by the pigeonhole principle, there exists $u \neq v$ in $\mathcal{G}^{(0)}$ such that $f_{i}(u)=f_{i}(v)$ for all $1 \leq i \leq n$, and thus $f(u)=f(v)$ for all $f \in A_{\mathbb{K}}(\mathcal{G})$. But $\mathcal{G}^{(0)}$ is Hausdorff, locally compact, and totally disconnected, so there is a compact open subset $U \subseteq \mathcal{G}^{(0)}$ with $u \in U$ and $v \notin U$. Since $\mathcal{G}^{(0)}$ is open in $\mathcal{G}$, it follows that $U$ is a compact open bisection in $\mathcal{G}$, so $\mathbf{1}_{U} \in A_{\mathbb{K}}(\mathcal{G})$. We arrive at a contradiction, because $\mathbf{1}_{U}(u) \neq \mathbf{1}_{U}(v)$. Therefore $\left|\mathcal{G}^{(0)}\right| \leq M^{n}<\infty$. A finite Hausdorff space is discrete, so $\mathcal{G}^{(0)}$ is discrete. As $\mathcal{G}$ is étale, it must also be discrete. Thus $\operatorname{dim}_{\mathbb{K}} A_{\mathbb{K}}(\mathcal{G})=n=|\mathcal{G}|$. Given that $\mathcal{G}$ is finite and discrete, it is isomorphic to a disjoint union of transitive groupoids (one for each orbit), each of which is isomorphic to the product of a transitive principal groupoid (with as many elements as the corresponding orbit), and a finite group (the isotropy group of that orbit). The expression giving the structure of $A_{\mathbb{K}}(\mathcal{G})$ follows from Propositions 1.23 and 1.25 .

1.6. Graded groupoids and graded Steinberg algebras. Just as the Steinberg algebra of a groupoid inherits an involution from the groupoid, so it can inherit a graded structure. Many well-studied examples of Steinberg algebras receive a canonical group-grading that comes from a grading on the groupoid itself. We first introduce the concepts and terminology of graded groupoids and graded algebras.

A standing assumption is that $\Gamma$ is a group with identity $\varepsilon$. A ring $A$ is called a $\Gamma$-graded ring if it decomposes as a direct sum of additive subgroups $A=\bigoplus_{\gamma \in \Gamma} A_{\gamma}$ such that $A_{\gamma} A_{\delta} \subseteq A_{\gamma \delta}$ for every $\gamma, \delta \in \Gamma$. The meaning of $A_{\gamma} A_{\delta}$ is the additive subgroup generated by all products $a b$ where $a \in A_{\gamma}, b \in A_{\delta}$. The additive group $A_{\gamma}$ is called the $\gamma$-component of $A$. The elements of $\bigcup_{\gamma \in \Gamma} A_{\gamma}$ in a graded ring $A$ are called homogeneous elements. The nonzero elements of $A_{\gamma}$ are called $\gamma$-homogeneous, and we write $\operatorname{deg}(a)=\gamma$ for $a \in A_{\gamma} \backslash\{0\}$. When it is clear from context that a ring $A$ is graded by the group $\Gamma$, we simply say that $A$ is a graded ring. If $A$ is an $R$-algebra, then $A$ is called a graded algebra if it is a graded ring and each $A_{\gamma}$ is an $R$-submodule.

An ideal $I \subseteq A$ is a graded ideal if $I \subseteq \sum_{\gamma \in \Gamma} I \cap A_{\gamma}$. Graded left ideals, graded right ideals, graded subrings, and graded subalgebras are defined in a similar manner. If $H$ is a set of homogeneous elements in $A$, the ideal generated by $H$ is a graded ideal. Likewise, the left and right ideals generated by $H$ are graded. A graded homomorphism of $\Gamma$-graded rings is a homomorphism $f: A \rightarrow B$ such that $f\left(A_{\gamma}\right) \subseteq B_{\gamma}$ for every $\gamma \in \Gamma$. Finally, we say that a $\Gamma$-graded ring $A$ has homogeneous local units (or graded local units) if $A$ is locally unital, and the set of local units can be chosen to be a subset of $A_{\varepsilon}$.

A topological groupoid $\mathcal{G}$ is called $\Gamma$-graded if it can be partitioned by clopen subsets $\mathcal{G}=\bigsqcup_{\gamma \in \Gamma} \mathcal{G}_{\gamma}$, such that $\mathcal{G}_{\gamma} \mathcal{G}_{\delta} \subseteq \mathcal{G}_{\gamma \delta}$ for every $\gamma, \delta \in \Gamma$. Equivalently $\mathcal{G}$ is $\Gamma$-graded if there is a continuous homomorphism $\kappa: \mathcal{G} \rightarrow \Gamma$. We can show the definitions are equivalent by setting $\mathcal{G}_{\gamma}=\kappa^{-1}(\{\gamma\})$. If $\kappa: \mathcal{G} \rightarrow \Gamma$ defines the grading on $\mathcal{G}$, we call it the degree map. We use the notation $\mathcal{G}_{\gamma} x=\mathcal{G}_{\gamma} \cap \mathcal{G} x$ and $x \mathcal{G}_{\gamma}=x \mathcal{G} \cap \mathcal{G}_{\gamma}$ for $x \in \mathcal{G}^{(0)}$ and $\gamma \in \Gamma$.

We say a subset $X \subseteq \mathcal{G}$ is $\gamma$-homogeneous if $X \subseteq \mathcal{G}_{\gamma}$. Obviously, the unit space is $\varepsilon$-homogeneous and if $X$ is $\gamma$-homogeneous then $X^{-1}$ is $\gamma^{-1}$-homogeneous. Moreover, $\mathcal{G}_{\gamma}{ }^{-1}=\mathcal{G}_{\gamma^{-1}}$ for all $\gamma \in \Gamma$. For a $\Gamma$-graded ample groupoid, we write $B_{\gamma}^{\text {co }}(\mathcal{G})$ for the set of all $\gamma$-homogeneous compact open bisections of $\mathcal{G}$. For the set of all homogeneous compact open bisections, we use the notation:

$$
B_{*}^{\mathrm{co}}(\mathcal{G})=\bigcup_{\gamma \in \Gamma} B_{\gamma}^{\mathrm{co}}(\mathcal{G}) \subseteq B^{\mathrm{co}}(\mathcal{G})
$$

In Proposition 1.10, we proved that $B^{\mathrm{co}}(\mathcal{G})$ is an inverse semigroup, and it is readily apparent that $B_{*}^{\mathrm{co}}(\mathcal{G})$ is an inverse subsemigroup of $B^{\mathrm{co}}(\mathcal{G})$. In addition, $B_{*}^{\mathrm{co}}(\mathcal{G})$ is a base of compact open bisections for $\mathcal{G}$. Indeed, since $B^{\mathrm{co}}(\mathcal{G})$ is a base for $\mathcal{G}$, it suffices to show that every $B \in B^{\mathrm{co}}(\mathcal{G})$ is a union of sets in $B_{*}^{\mathrm{co}}(\mathcal{G})$. This is almost trivial, for if $B \in B^{\mathrm{co}}(\mathcal{G})$ then $B=\bigcup_{\gamma \in \Gamma} B \cap \mathcal{G}_{\gamma}$ and $B \cap \mathcal{G}_{\gamma} \in B_{\gamma}^{\mathrm{co}}(\mathcal{G})$. The next two results are from [32, Lemma 3.1]. 
Proposition 1.33. If $\mathcal{G}=\bigsqcup_{\gamma \in \Gamma} \mathcal{G}_{\gamma}$ is a $\Gamma$-graded ample groupoid, then $A_{R}(\mathcal{G})=\bigoplus_{\gamma \in \Gamma} A_{R}(\mathcal{G})_{\gamma}$ is a $\Gamma$-graded algebra with homogeneous local units, where:

$$
A_{R}(\mathcal{G})_{\gamma}=\left\{f \in A_{R}(\mathcal{G}) \mid \operatorname{supp} f \subseteq \mathcal{G}_{\gamma}\right\} \quad \text { for all } \gamma \in \Gamma .
$$

Proof. From Proposition 1.13, it follows that

$$
A_{R}(\mathcal{G})=\operatorname{span}_{R}\left\{\mathbf{1}_{B} \mid B \in B_{*}^{\mathrm{co}}(\mathcal{G})\right\}=\sum_{\gamma \in \Gamma} \operatorname{span}_{R}\left\{\mathbf{1}_{B} \mid B \in B_{\gamma}^{\mathrm{co}}(\mathcal{G})\right\}=\sum_{\gamma \in \Gamma} A_{R}(\mathcal{G})_{\gamma} .
$$

It is clear that $A_{R}(\mathcal{G})_{\gamma} \cap\left(\sum_{\delta \neq \gamma} A_{R}(\mathcal{G})_{\delta}\right)=\{0\}$ for all $\gamma \in \Gamma$, so we have $A_{R}(\mathcal{G})=\bigoplus_{\gamma \in \Gamma} A_{R}(\mathcal{G})_{\gamma}$. Now for all $f \in A_{R}(\mathcal{G})_{\gamma}$ and $g \in A_{R}(\mathcal{G})_{\delta}$, we have $\operatorname{supp}(f * g) \subseteq \operatorname{supp}(f) \operatorname{supp}(g) \subseteq \mathcal{G}_{\gamma} \mathcal{G}_{\delta} \subseteq \mathcal{G}_{\gamma \delta}$, and thus $f * g \in A_{R}(\mathcal{G})_{\gamma \delta}$. Therefore $A_{R}(\mathcal{G})_{\gamma} * A_{R}(\mathcal{G})_{\delta} \subseteq A_{R}(\mathcal{G})_{\gamma \delta}$. It follows from Proposition 1.20, and the fact that $\mathcal{G}^{(0)} \subseteq \mathcal{G}_{\varepsilon}$, that $A_{R}(\mathcal{G})$ has homogeneous local units.

Lemma 1.34. If $\mathcal{G}$ is a $\Gamma$-graded Hausdorff ample groupoid, every $f \in A_{R}(\mathcal{G})$ can be expressed as a finite sum $f=\sum_{i=1}^{n} r_{i} \mathbf{1}_{B_{i}}$, where $r_{1}, \ldots, r_{n} \in R$, and $B_{1}, \ldots, B_{n} \in B_{*}^{\mathrm{co}}(\mathcal{G})$ are mutually disjoint.

Proof. Since $\mathcal{G}$ is Hausdorff, every homogeneous compact open bisection is closed, so $B_{*}^{\text {co }}(\mathcal{G})$ is closed under finite intersections and relative complements. The statement now follows from Proposition 1.19.

Example 1.35. Recall, from Example $1.3(\mathrm{~d})$, the definition of the transformation groupoid $G \times X$, associated to a group $G$ and a $G$-set $X$. Now assume that $X$ is a locally compact, totally disconnected, Hausdorff topological space, and for each $g \in G$ the map $\rho_{g}: X \rightarrow X, \rho_{g}(x)=g \cdot x$, is continuous. If we assign the discrete topology to $G$ and the product topology to $G \times X$, then $G \times X$ is an ample groupoid. It is easy to verify that this is a $G$-graded groupoid with homogeneous components $(G \times X)_{g}=\{g\} \times X$ for all $g \in G$. The Steinberg algebra of $G \times X$ turns out (see [17]) to be the skew group $\operatorname{ring} C_{R}(X) \star G$, associated to a certain action of $G$ on $C_{R}(X)$, canonically induced by the action of $G$ on $X$.

One can generalise this example quite profitably, by replacing the group action with something more general called a partial group action (see [40, Definition 2.1]). In doing so, one obtains a class of algebras so general that it includes all Leavitt path algebras (see [41, Theorem 3.3]) and other interesting things, like the partial group algebras that were studied in [36] and [45].

\section{The PATH SPACE AND BOUNDARY PATH GROUPOID OF A GRAPH}

Part 2 is structured as follows. In $\S 2.1$, we define directed graphs and introduce some terminology. In $\S 2.2$, we introduce a topological space called the path space of a graph. The path space of a graph is the set of all finite and infinite paths, with a topology described explicitly by a base of open sets. Generalising [69, Theorem 2.1], we prove in Theorem 2.4 that for graphs of any cardinality, the path space is locally compact and Hausdorff. We also determine which graphs have a second-countable, first-countable, or $\sigma$-compact path space. In $\S 2.3$, we use the path space (or more precisely, a closed subspace called the boundary path space) to define the boundary path groupoid associated to a graph. We prove it is ample and study its local structure from a topological and an algebraic point of view.

Remark 2.1. Perhaps as an artefact of its history, many fundamental properties of the boundary path groupoid were absorbed into folklore. Some proofs were never written, and others were written at a higher level of generality, and not all in one place, making them difficult to relate back to our present needs. For instance, we could not find a complete proof that the boundary path groupoid is an ample groupoid, even though this fact was used in all the early papers that pioneered the use of groupoid methods for Leavitt path algebras [28, 29, 32]. The groupoid approach to Leavitt path algebras is particularly wellsuited, compared to traditional, purely algebraic methods, for dealing with graphs of large cardinalities. Therefore, it is important to make sure that the theorems used to justify these methods can be proved without assuming graphs are countable. This is something that we achieve here, in Theorem 2.4 and Theorem 2.17. 
2.1. Graphs. In this section, we introduce the necessary terminology and conventions pertaining to graphs. We always use the word graph to mean a directed graph, defined as follows.

Definition 2.2. A graph is a system $E=\left(E^{0}, E^{1}, r, s\right)$, where $E^{0}$ is a set whose elements are called vertices, $E^{1}$ is a set whose elements are called edges, $r: E^{1} \rightarrow E^{0}$ is a map that associates a range to every edge, and $s: E^{1} \rightarrow E^{0}$ is a map that associates a source to every edge.

A countable graph is one where $E^{0}$ and $E^{1}$ are countable sets. A row-finite (resp., row-countable) graph is one in which $s^{-1}(v)$ is finite (resp., countable) for every $v \in E^{0}$. If $e$ is an edge with $s(e)=v$ and $r(e)=w$ then we say that $v$ emits $e$ and $w$ receives $e$. A sink is a vertex that emits no edges and an infinite emitter is a vertex that emits infinitely many edges. If $v \in E^{0}$ is either a sink or an infinite emitter (that is, $s^{-1}(v)$ is either empty or infinite) then $v$ is called singular, and if $v$ is not singular then it is called regular. A vertex that neither receives nor emits any edges is called an isolated vertex.

A finite path is a finite sequence of edges $\alpha=\alpha_{1} \alpha_{2} \ldots \alpha_{n}$ such that $r\left(\alpha_{i}\right)=s\left(\alpha_{i+1}\right)$ for all $i=1, \ldots, n-1$. The length of the path $\alpha$ is $|\alpha|=n$. Reusing notation and terminology, we shall say that $s(\alpha)=s\left(\alpha_{1}\right)$ is the source of the path, and $r(\alpha)=r\left(\alpha_{n}\right)$ is the range of the path. By convention, vertices $v \in E^{0}$ are regarded as finite paths of zero length, with $r(v)=s(v)=v$. If $v, w \in E^{0}$, we write $v \geq w$ if there exists a finite path $\alpha$ with $s(\alpha)=v$ and $r(\alpha)=w$. If a finite path $\alpha$ of positive length satisfies $r(\alpha)=s(\alpha)=v$, then $\alpha$ is called a closed path based at $v$. A closed path $\alpha$ with the property that none of the vertices $s\left(\alpha_{1}\right), \ldots, s\left(\alpha_{|\alpha|}\right)$ are repeated is called a cycle, and a graph that has no cycles is called acyclic. An exit for a finite path $\alpha$ is an edge $f \in E^{1}$ with $s(f)=s\left(\alpha_{i}\right)$ for some $1 \leq i \leq|\alpha|$, but $f \neq \alpha_{i}$.

An infinite path is, predictably, an infinite sequence of edges $p=p_{1} p_{2} p_{3} \ldots$ such that $r\left(p_{i}\right)=s\left(p_{i+1}\right)$ for $i=1,2, \ldots$. Again, $s(p)=s\left(p_{1}\right)$ is called the source of the infinite path $p$. We let $|p|=\infty$ if $p$ is an infinite path. We use the notation $E^{\star}$ for the set of finite paths (including vertices), and $E^{\infty}$ for the set of infinite paths.

Paths can be concatenated if their range and source agree. If $\alpha, \beta \in E^{\star}$ have positive length and $r(\alpha)=$ $s(\beta)$, then $\alpha \beta=\alpha_{1} \ldots \alpha_{|\alpha|} \beta_{1} \ldots \beta_{|\beta|} \in E^{\star}$. If $p \in E^{\infty}$ has $r(\alpha)=s(p)$, then $\alpha p=\alpha_{1} \ldots \alpha_{|\alpha|} p_{1} p_{2} \ldots \in$ $E^{\infty}$. If $v \in E^{0}$ and $x \in E^{\star} \cup E^{\infty}$ has $s(x)=v$, then $v x=x$ by convention. Likewise, if $\alpha \in E^{\star}$ has $r(\alpha)=v$ then $\alpha v=\alpha$. If $\alpha \in E^{\star}, x \in E^{\star} \cup E^{\infty}$, and $x=\alpha x^{\prime}$ for some $x^{\prime} \in E^{\star} \cup E^{\infty}$, then we say that $\alpha$ is an initial subpath of $x$. In particular, $s(\alpha)$ is considered an initial subpath of $\alpha$.

Let $E_{\mathrm{sing}}^{0}=\left\{v \in E^{0} \mid v\right.$ is singular $\}$ and $E_{\mathrm{reg}}^{0}=\left\{v \in E^{0} \mid v\right.$ is regular $\}$. Using the terminology of [69], we define the set of boundary paths as

$$
\partial E=E^{\infty} \cup\left\{\alpha \in E^{\star} \mid r(\alpha) \in E_{\text {sing }}^{0}\right\}
$$

We employ the following notation from now on:

$$
\begin{aligned}
& v E^{1}=\left\{e \in E^{1} \mid s(e)=v\right\}, \\
& v E^{\infty}=\left\{p \in E^{\infty} \mid s(p)=v\right\}, \\
& E^{\star} \times_{r} E^{\star}=\left\{(\alpha, \beta) \in E^{\star} \times E^{\star} \mid r(\alpha)=r(\beta)\right\} .
\end{aligned}
$$

$$
\begin{aligned}
& v E^{\star}=\left\{\alpha \in E^{\star} \mid s(\alpha)=v\right\}, \\
& v \partial E=\{x \in \partial E \mid s(x)=v\},
\end{aligned}
$$

2.2. The path space of a graph. Throughout this section, assume $E=\left(E^{0}, E^{1}, r, s\right)$ is an arbitrary graph. The path space of $E$ is $E^{\star} \cup E^{\infty}$, the set of all finite and infinite paths, and the boundary path space is $\partial E$, the set of paths that are either infinite or end at a singular vertex. We now set out to define a suitable topology on the path space. For a finite path $\alpha \in E^{\star}$, we define the cylinder set

$$
C(\alpha)=\left\{\alpha x \mid x \in E^{\star} \cup E^{\infty}, r(\alpha)=s(x)\right\} \subseteq E^{\star} \cup E^{\infty} .
$$

It is easy to see that the intersection of two cylinders is either empty or a cylinder. Indeed, if $x \in$ $C(\alpha) \cap C(\beta)$ then $x=\alpha y=\beta z$ for some $y, z \in E^{\star} \cup E^{\infty}$. If $|\alpha| \leq|\beta|$ then $\alpha$ is an initial subpath of $\beta$, 
implying $C(\beta) \subseteq C(\alpha)$. In symbols:

$$
C(\alpha) \cap C(\beta)= \begin{cases}C(\beta) & \text { if } \alpha \text { is an initial subpath of } \beta \\ C(\alpha) & \text { if } \beta \text { is an initial subpath of } \alpha \\ \emptyset & \text { otherwise. }\end{cases}
$$

This is all we need to conclude that the collection of cylinder sets is a base for a topology on $E^{\star} \cup E^{\infty}$. As the authors of [50] have stated, the subspace $E^{\infty} \subseteq E^{\star} \cup E^{\infty}$ with the cylinder set topology is homeomorphic (in the canonical way) to a subspace of $\prod_{n=1}^{\infty} E^{1}$, where $E^{1}$ is discrete and the product has the product topology. In particular, the cylinder sets generate a Hausdorff topology on $E^{\infty}$, and if $E$ is row-finite, that topology is locally compact. However, the cylinder set topology generated by the sets (2.1) is not Hausdorff (or even $T_{1}$ ) on the whole set $E^{\star} \cup E^{\infty}$, because a finite path cannot be separated from a proper initial subpath. In order to have enough open sets in hand for a Hausdorff topology, we define a base of open sets called generalised cylinder sets:

$$
C(\alpha, F)=C(\alpha) \backslash \bigcup_{e \in F} C(\alpha e) ; \quad \alpha \in E^{\star}, F \subseteq r(\alpha) E^{1} \text { is finite. }
$$

We shall write $F \subseteq_{\text {finite }} v E^{1}$ to mean that $F$ is a finite subset of $v E^{1}$. The next lemma (a generalisation of [50, Lemma 2.1]) shows that the collection of generalised cylinders is closed under intersections, so it is a base for a topology on $E^{\star} \cup E^{\infty}$. With the generalised cylinder set topology on $E^{\star} \cup E^{\infty}$, every finite path is an isolated point unless its range is an infinite emitter.

Lemma 2.3. If $\alpha, \beta \in E^{\star},|\alpha| \leq|\beta|, F \subseteq_{\text {finite }} r(\alpha) E^{1}$, and $H \subseteq$ finite $r(\beta) E^{1}$, then

$$
C(\alpha, F) \cap C(\beta, H)= \begin{cases}C(\beta, F \cup H) & \text { if } \beta=\alpha \\ C(\beta, H) & \text { if } \exists \delta \in E^{\star},|\delta| \geq 1, \beta=\alpha \delta, \text { and } \delta_{1} \notin F \\ \emptyset & \text { otherwise. }\end{cases}
$$

Proof. By definition of $C(\alpha, F)$ and $C(\beta, H)$, we have

$$
C(\alpha, F) \cap C(\beta, H)=C(\alpha) \cap C(\beta) \backslash\left(\bigcup_{e \in F} C(\alpha e) \cup \bigcup_{e \in H} C(\beta e)\right) .
$$

If $\beta=\alpha$, the right hand side of $(2.3)$ is $C(\beta, F \cup H)$. If $\beta=\alpha \delta(|\delta| \geq 1)$ and $\delta_{1} \notin F$ then $C(\beta) \cap C(\alpha)=$ $C(\beta)$ does not meet $\bigcup_{e \in F} C(\alpha e)$, so the right hand side of $(2.3)$ is $C(\beta, H)$. If $\beta=\alpha \delta$ and $\delta_{1} \in F$, then $C(\beta) \cap C(\alpha)=C(\beta)=C\left(\alpha \delta_{1} \ldots \delta_{|\delta|}\right) \subseteq C\left(\alpha \delta_{1}\right) \subseteq \bigcup_{e \in F} C(\alpha e)$, so the right hand side of (2.3) is empty. If $\alpha$ is not an initial subpath of $\beta$ then $C(\alpha) \cap C(\beta)=\emptyset$.

To apply Steinberg's theory from Part 1, it is critical that the induced topology on the boundary path space $\partial E \subseteq E^{\star} \cup E^{\infty}$ is locally compact and Hausdorff. We proceed by proving that the topology on the path space $E^{\star} \cup E^{\infty}$, generated by the base in (2.2), is locally compact and Hausdorff, and that $\partial E$ is closed in $E^{\star} \cup E^{\infty}$. As it were, this base is well-chosen: the basic open sets themselves are compact in the Hausdorff topology that they generate.

The proof of the theorem below is essentially the same as [69, Theorem 2.1], just written slightly differently so that it does not use any assumptions of countability. The main idea is to equip $\mathbb{P}\left(E^{\star}\right)$, i.e., the power set of $E^{\star}$, with a compact Hausdorff topology, and show that $E^{\star} \cup E^{\infty}$ is homeomorphic to a locally compact subspace $\mathbb{S} \subset \mathbb{P}\left(E^{\star}\right)$.

Theorem 2.4. The collection (2.2) of generalised cylinder sets is a base of compact open sets for a locally compact Hausdorff topology on $E^{\star} \cup E^{\infty}$.

Proof. Let $\{0,1\}$ have the discrete topology. The product space $\{0,1\}^{E^{\star}}$ is compact by Tychonoff's Theorem, and Hausdorff because products preserve the Hausdorff property. There is a canonical bijection from $\mathbb{P}\left(E^{\star}\right)$ to $\{0,1\}^{E^{\star}}$, which transfers a compact Hausdorff topology to $\mathbb{P}\left(E^{\star}\right)$. For the first part of the 
proof, we work entirely in the space $\mathbb{P}\left(E^{\star}\right)$. The topology on $\mathbb{P}\left(E^{\star}\right)$, by definition, is generated by the base of open sets

$$
\left\{[P, N] \mid P, N \subseteq \text { finite } E^{\star}\right\}
$$

where we define

$$
[P, N]=\left\{A \in \mathbb{P}\left(E^{\star}\right) \mid P \subseteq A, N \subseteq E^{\star} \backslash A\right\} .
$$

Note that $[P, N]=\emptyset$ if $P \cap N \neq \emptyset$.

Define the subspace $\mathbb{S} \subset \mathbb{P}\left(E^{\star}\right)$ to be the set of subsets $A \subseteq E^{\star}$ such that:

- $A \neq \emptyset$ and for all $\alpha \in A$, every initial subpath of $\alpha$ is in $A$;

- For every $0 \leq n<\infty$, there is at most one path of length $n$ in $X$.

We claim that $\mathbb{S} \cup\{\emptyset\}$ is closed in $\mathbb{P}\left(E^{\star}\right)$. Suppose $A \in \mathbb{P}\left(E^{\star}\right) \backslash(\mathbb{S} \cup\{\emptyset\})$. If $A$ contains two distinct paths $\alpha$ and $\beta$ of the same length, then $[\{\alpha, \beta\}, \emptyset]$ is open, contains $A$, and does not meet $\mathbb{S} \cup\{\emptyset\}$. If there is some $\alpha \in A$ and an initial subpath $\beta$ of $\alpha$ such that $\beta \notin A$, then $[\{\alpha\},\{\beta\}]$ is open, contains $A$, and does not meet $\mathbb{S} \cup\{\emptyset\}$. Failing this, $A \in \mathbb{S} \cup\{\emptyset\}$, which we assumed is false. Therefore $\mathbb{S} \cup\{\emptyset\}$ is closed in $\mathbb{P}\left(E^{\star}\right)$, which implies it is compact.

We now work out what the subspace topology is on $\mathbb{S}$. Let $P, N \subseteq$ finite $E^{\star}$. If $[P, N] \cap \mathbb{S} \neq \emptyset$ then $P$ contains a unique path $\rho$ of maximal length (because of the way $\mathbb{S}$ is defined) and $[P, N] \cap \mathbb{S}=\left[\{\rho\}, N^{\prime}\right] \cap \mathbb{S}$ where

$$
N^{\prime}=\{\eta \in N \mid \rho \text { is an initial subpath of } \eta\} .
$$

Therefore, the topology on $\mathbb{S}$ is generated by basic open sets of the form $\left[\{\rho\}, N^{\prime}\right] \cap \mathbb{S}$ where $\rho \in E^{\star}$ and $N^{\prime} \subseteq E^{\star}$ is a finite set of paths that are proper extensions of $\rho$.

Note that $\mathbb{S}=\bigsqcup_{v \in E^{0}}[\{v\}, \emptyset] \cap \mathbb{S}$. For each $v \in E^{0}$, the set $[\{v\}, \emptyset]$ is closed in $\mathbb{P}\left(E^{\star}\right)$ because $\mathbb{P}\left(E^{\star}\right) \backslash$ $[\{v\}, \emptyset]=[\emptyset,\{v\}]$ is open. Since $[\{v\}, \emptyset] \cap \mathbb{S}=[\{v\}, \emptyset] \cap(\mathbb{S} \cup\{\emptyset\})$ and $\mathbb{S} \cup\{\emptyset\}$ is closed in $\mathbb{P}\left(E^{\star}\right)$, we have that $[\{v\}, \emptyset] \cap \mathbb{S}$ is closed in $\mathbb{P}\left(E^{\star}\right)$, and therefore compact. This proves that $\mathbb{S}$ is locally compact, because it is Hausdorff and every point has a compact neighbourhood.

Now we show that $E^{\star} \cup E^{\infty}$ is homeomorphic to $\mathbb{S}$. Define the map $\Psi: E^{\star} \cup E^{\infty} \rightarrow \mathbb{S}$,

$$
\Psi(x)=\left\{\nu \in E^{\star} \mid \nu \text { is an initial subpath of } x\right\} .
$$

It is clear that $\Psi$ is a bijection. Let $\rho \in E^{\star}$ and let $N^{\prime} \subseteq E^{\star}$ be a finite set of paths that properly extend $\rho$. Then

$$
\Psi^{-1}\left(\left[\{\rho\}, N^{\prime}\right] \cap \mathbb{S}\right)=C(\rho) \backslash \bigcup_{\rho \beta \in N^{\prime}} C(\rho \beta)=\bigcap_{\rho \beta \in N^{\prime}} C(\rho) \backslash C(\rho \beta)
$$

It is not difficult to see that for each $\rho \beta \in N^{\prime}$, the set

$$
C(\rho) \backslash C(\rho \beta)=C\left(\rho,\left\{\beta_{1}\right\}\right) \cup C\left(\rho \beta_{1},\left\{\beta_{2}\right\}\right) \cup \cdots \cup C\left(\rho \beta_{|\beta|-1},\left\{\beta_{|\beta|}\right\}\right)
$$

is open. Therefore $\Psi^{-1}\left(\left[\{\rho\}, N^{\prime}\right] \cap \mathbb{S}\right)$ is open in $E^{\star} \cup E^{\infty}$. Consequently, $\Psi$ is continuous. If $\alpha \in E^{\star}$ and $F \subseteq$ finite $r(\alpha) E^{1}$, then $C(\alpha, F)$ is mapped to an open set in $\mathbb{S}$ :

$$
\Psi(C(\alpha, F))=\left[\{\alpha\}, N^{\prime}\right] \cap \mathbb{S}
$$

where $N^{\prime}=\{\alpha e \mid e \in F\}$. It follows that $\Psi$ is a homeomorphism and $E^{\star} \cup E^{\infty}$ is Hausdorff.

Since we showed that $[\{v\}, \emptyset] \cap \mathbb{S}$ is compact, it follows that $C(v)=\Psi^{-1}([\{v\}, \emptyset] \cap \mathbb{S})$ is compact, for all $v \in E^{0}$. To show that $C(\alpha)$ is compact for all $\alpha \in E^{\star}$, we proceed by induction on the length of $\alpha$. If $e \in E^{1}$, then $C(s(e)) \backslash C(e)=C(s(e),\{e\})$ is a basic open set, so $C(e)$ is closed in $C(s(e))$, hence compact. Assume $C(\alpha)$ is compact for any $\alpha \in E^{\star}$ with $|\alpha|=n$. If $\mu \in E^{\star}$ has $|\mu|=n+1$ then let $\mu^{\prime}=\mu_{1} \mu_{2} \ldots \mu_{n}$. We have that $C\left(\mu^{\prime}\right) \backslash C(\mu)=C\left(\mu^{\prime},\left\{\mu_{n+1}\right\}\right)$ is a basic open set, so $C(\mu)$ is closed in $C\left(\mu^{\prime}\right)$, hence compact. By induction, $C(\alpha)$ is compact for arbitrary $\alpha \in E^{\star}$. Finally, if $F \subseteq$ finite $r(\alpha) E^{1}$ then $C(\alpha) \backslash C(\alpha, F)=\bigcup_{e \in F} C(\alpha e)$ is open, so $C(\alpha, F)$ is compact. 
Recall that a topological space is called second-countable if it has a countable base, first-countable if every point has a countable neighbourhood base, and $\sigma$-compact if it is a countable union of compact subsets.

Theorem 2.5. The path space $E^{\star} \cup E^{\infty}$ is:

(1) second-countable if and only if $E$ is a countable graph;

(2) first-countable if and only if $E$ is a row-countable graph;

(3) $\sigma$-compact if and only if $E^{0}$ is countable.

Proof. (1) If $E$ is a countable graph (i.e., $E^{0} \cup E^{1}$ is countable) then $E^{\star}$ is countable. The base of open sets (2.2) is countable too, because there are only countably many pairs $(\alpha, F)$ where $\alpha \in E^{\star}$ and $F \subseteq$ finite $r(\alpha) E^{1}$. This proves the topology is second-countable. Conversely, if one of $E^{0}$ or $E^{1}$ is uncountable, then one of $\left\{C(v) \mid v \in E^{0}\right\}$ or $\left\{C(e) \mid e \in E^{1}\right\}$ is an uncountable set of pairwise disjoint open sets, so $E^{\star} \cup E^{\infty}$ is not second-countable.

(2) Notice that the following sets are neighbourhood bases at $\alpha \in E^{\star}$ and $p \in E^{\infty}$ respectively:

$$
\mathcal{N}_{\alpha}=\left\{C(\alpha, F) \mid F \subseteq_{\text {finite }} r(\alpha) E^{1}\right\}, \quad \mathcal{N}_{p}=\left\{C\left(p_{1} \ldots p_{m}\right) \mid m \geq 1\right\} .
$$

Regardless of the graph, $\mathcal{N}_{p}$ is countable for every $p \in E^{\infty}$. If a finite path $\alpha \in E^{\star}$ has the property that $r(\alpha) E^{1}$ is countable, then $\mathcal{N}_{\alpha}$ is countable, because there are only countably many finite subsets $F$ of $r(\alpha) E^{1}$. So, for every row-countable graph $E$, the path space $E^{\star} \cup E^{\infty}$ is first-countable. Conversely, suppose there exists $v \in E^{0}$ such that $v E^{1}$ is uncountable. Towards a contradiction, assume $v$ has a countable neighbourhood base $\mathcal{B}_{v}=\left\{B_{1}, B_{2}, \ldots,\right\}$. By replacing $B_{n}$, for all $n \geq 1$, with a set of the form $C\left(v, F_{n}\right) \subseteq B_{n}$, where $F_{n} \subseteq$ finite $v E^{1}$, we have a countable neighbourhood base for $v$ of the form $\mathcal{C}_{v}=\left\{C\left(v, F_{1}\right), C\left(v, F_{2}\right), \ldots\right\}$. Since $\bigcup_{n=1}^{\infty} F_{n}$ is countable, one can choose $e \in v E^{1} \backslash \bigcup_{n=1}^{\infty} F_{n}$. Then every neighbourhood of $v$ contains $e$, which is absurd, because the space is Hausdorff. Therefore $E^{\star} \cup E^{\infty}$ is first-countable if and only if $E$ is row-countable.

(3) If $E^{0}$ is countable then the path space is $\sigma$-compact, because $E^{\star} \cup E^{\infty}=\bigcup_{v \in E^{0}} C(v)$ and $C(v)$ is compact for every $v \in E^{0}$, by Theorem 2.4. For the converse, suppose $E^{\star} \cup E^{\infty}$ is $\sigma$-compact. Then there is a sequence of compact subsets $\left(K_{n}\right)_{1}^{\infty}$ such that $E^{\star} \cup E^{\infty}=\bigcup_{n=1}^{\infty} K_{n}$. Each $K_{n}$ is compact, so it can be covered by a finite subcover of $\left\{C(v) \mid v \in E^{0}\right\}$, implying that there is a countable set $S \subseteq E^{0}$ such that $E^{\star} \cup E^{\infty}=\bigcup_{v \in S} C(v)$. But this implies $S=E^{0}$ because $C(v)$ and $C(w)$ are disjoint unless $v=w$.

We now prove an easy fact that forms a bridge to the next section, where we shall construct a groupoid with unit space $\partial E=E^{\infty} \cup\left\{\alpha \in E^{\star} \mid r(\alpha) \in E_{\text {sing }}^{0}\right\}$.

Proposition 2.6. The boundary path space $\partial E$ is closed in $E^{\star} \cup E^{\infty}$.

Proof. The complement of $\partial E$ consists of isolated points. Indeed, if $\mu \in\left(E^{\star} \cup E^{\infty}\right) \backslash \partial E$, then $r(\mu)$ is a regular vertex, and $C\left(\mu, r(\mu) E^{1}\right)=\{\mu\}$ is open in $E^{\star} \cup E^{\infty}$.

An immediate consequence of Theorem 2.4 and Proposition 2.6 is that $\partial E$ is a locally compact Hausdorff space with the base of compact open sets:

$$
Z(\alpha, F)=C(\alpha, F) \cap \partial E ; \quad \alpha \in E^{\star}, F \subseteq_{\text {finite }} r(\alpha) E^{1} .
$$

For $\alpha \in E^{\star}$, we define $Z(\alpha)=Z(\alpha, \emptyset)$, which is the same as $Z(\alpha)=C(\alpha) \cap \partial E$. As it were, the sets $Z(\alpha, F)$ are very rarely empty. In particular, $Z(\alpha) \neq \emptyset$ for all $\alpha \in E^{\star}$; in other words, every finite path can be extended to a boundary path.

Lemma 2.7. Let $\alpha \in E^{\star}$ and let $F \subseteq$ finite $r(\alpha) E^{1}$. Then $Z(\alpha, F)=\emptyset$ if and only if $r(\alpha)$ is a regular vertex and $F=r(\alpha) E^{1}$. 
Proof. $(\Rightarrow)$ Assume $Z(\alpha, F)=\emptyset$. If $r(\alpha)$ were a singular vertex then it would imply $\alpha \in Z(\alpha, F)$. Therefore $r(\alpha)$ is regular, so $r(\alpha) E^{1} \neq \emptyset$. Towards a contradiction, assume $F$ is a proper subset of $r(\alpha) E^{1}$. Then there exists some $x_{1} \in r(\alpha) E^{1} \backslash F$. Assume that we have a path $x_{1} x_{2} \ldots x_{n} \in r(\alpha) E^{\star}$. If $r\left(x_{n}\right)$ is a sink, let $x=x_{1} \ldots x_{n}$. Otherwise, let $x_{n+1} \in r\left(x_{n}\right) E^{1}$. Inductively, this constructs $x \in r(\alpha) \partial E$ such that $\alpha x \in Z(\alpha, F)$. Since this is a contradiction, it proves $F=r(\alpha) E^{1}$.

$(\Leftarrow)$ If $r(\alpha)$ is regular, then $Z(\alpha)=\bigcup_{e \in r(\alpha) E^{1}} Z(\alpha e)$, so $Z\left(\alpha, r(\alpha) E^{1}\right)=\emptyset$.

Theorem 2.8. The boundary path space $\partial E$ is:

(1) second-countable if and only if $E$ is a countable graph,

(2) first-countable if and only if $E$ is a row-countable graph, and

(3) $\sigma$-compact if and only if $E^{0}$ is countable.

Proof. Together with Lemma 2.7, the proof is almost identical to the relevant parts of Theorem 2.5.

2.3. The boundary path groupoid. In this section, we define the boundary path groupoid of a graph (see [32, Example 2.1]) and investigate some of its algebraic and topological properties. Throughout, let $E=\left(E^{0}, E^{1}, r, s\right)$ be an arbitrary graph.

Define the one-sided shift map $\sigma: \partial E \backslash E^{0} \rightarrow \partial E$ as follows:

$$
\sigma(x)= \begin{cases}r(x) & \text { if } x \in E^{\star} \cap \partial E \text { and }|x|=1 \\ x_{2} \ldots x_{|x|} & \text { if } x \in E^{\star} \cap \partial E \text { and }|x| \geq 2 \\ x_{2} x_{3} \ldots & \text { if } x \in E^{\infty}\end{cases}
$$

The $n$-fold composition $\sigma^{n}$ is defined on paths of length $\geq n$ and we understand that $\sigma^{0}: \partial E \rightarrow \partial E$ is the identity map.

Definition 2.9. Let $k$ be an integer and let $x, y \in \partial E$. We say that $x$ and $y$ are tail equivalent with lag $k$, written $x \sim_{k} y$, if there exists some $n \geq \max \{0, k\}$ such that

$$
\sigma^{n}(x)=\sigma^{n-k}(y) \text {. }
$$

If an integer $k$ exists such that $x \sim_{k} y$, we say that $x$ and $y$ are tail equivalent, and write $x \sim y$.

An equivalent definition is that $x \sim_{k} y$ if there exists $(\alpha, \beta) \in E^{\star} \times_{r} E^{\star}$ and $z \in r(\alpha) \partial E$, such that $x=\alpha z, y=\beta z$, and $|\alpha|-|\beta|=k$. Something that is potentially counter-intuitive about these relations is that the lag is not necessarily unique: it is possible to have $x \sim_{k} y$ and $x \sim_{\ell} y$ even when $k \neq \ell$. It is straightforward to prove from the definition that for all $x, y, z \in \partial E$ :

$$
\begin{gathered}
x \sim_{0} x, \\
x \sim_{k} y \Longrightarrow y \sim_{-k} x, \\
x \sim_{k} y \text { and } y \sim_{\ell} z \Longrightarrow x \sim_{k+\ell} z, \\
x \sim_{k} y \Longrightarrow x, y \in E^{\star} \text { or } x, y \in E^{\infty} .
\end{gathered}
$$

This shows that $\sim$ is an equivalence relation on $\partial E$ that respects the partition between finite and infinite paths.

Definition 2.10. The boundary path groupoid of a graph $E$ is

$$
\begin{aligned}
\mathcal{G}_{E} & =\left\{(x, k, y) \mid x, y \in \partial E, x \sim_{k} y\right\} \\
& =\left\{(\alpha x,|\alpha|-|\beta|, \beta x) \mid(\alpha, \beta) \in E^{\star} \times_{r} E^{\star}, x \in r(\alpha) \partial E\right\}
\end{aligned}
$$

where a morphism $(x, k, y) \in \mathcal{G}_{E}$ has domain $y$ and codomain $x$. The composition of morphisms and their inverses are defined by the formulae:

$$
(x, k, y)(y, l, z)=(x, k+l, z), \quad(x, k, y)^{-1}=(y,-k, x) .
$$


The unit space is $\mathcal{G}_{E}^{(0)}=\{(x, 0, x) \mid x \in \partial E\}$, which we silently identify with $\partial E$ (see Remark 1.2). The orbits in $\partial E$ are tail equivalence classes.

Example 2.11. Consider this graph, called the rose with two petals:

$$
R_{2}={ }^{\bullet} \cdot{ }^{v}{ }^{f}
$$

A standard diagonal argument proves that $\partial R_{2}$ is an uncountable set. There are uncountably many orbits in $\partial R_{2}$, but the topology on $\partial R_{2}$ is second-countable and even metrisable. In fact, it can be shown that $\partial R_{2}$ is homeomorphic to the Cantor set $\{0,1\}^{\mathbb{N}}$.

A boundary path $p \in \partial E$ is called eventually periodic if it is of the form $p=\mu \epsilon \epsilon \ldots \in E^{\infty}$ where $\mu, \epsilon \in E^{\star}$ and $\epsilon$ is a closed path of positive length. The following result is [65, Proposition 4.2] except there appears to be a clash between our definitions of cycles and closed paths. We also prove it slightly more formally.

Proposition 2.12. If $E$ is a graph and $p \in \partial E$, then the isotropy group at $p$ is:

(1) infinite cyclic if $p$ is eventually periodic;

(2) trivial if $p$ is not eventually periodic.

Proof. (1) Assume $p=\mu \epsilon \epsilon \ldots \in E^{\infty}$ where $\mu, \epsilon \in E^{\star}, r(\mu)=s(\epsilon)=r(\epsilon)$, and assume $\epsilon$ is minimal in the sense that it has no initial subpath $\delta$ such that $\epsilon=\delta^{n}$ for some $n>1$. Let $(p, k, p) \in p\left(\mathcal{G}_{E}\right) p$ and suppose $k \geq 0$. Then $p \sim_{k} p$ implies that for all sufficiently large $n \geq 0$, we have $\sigma^{|\mu|+n|\epsilon|+k}(p)=\sigma^{|\mu|+n|\epsilon|}(p)$. This yields:

$$
\sigma^{|\mu|+n|\epsilon|+k}(p)=\sigma^{k}(\epsilon \epsilon \ldots)=\sigma^{|\mu|+n|\epsilon|}(p)=\epsilon \epsilon \ldots
$$

Let $m=k \bmod |\epsilon|$. Then $0 \leq m<|\epsilon|$ and

$$
\sigma^{k}(\epsilon \epsilon \ldots)=\sigma^{m}(\epsilon \epsilon \ldots)=\epsilon_{m+1} \ldots \epsilon_{|\epsilon|} \epsilon \epsilon \ldots=\epsilon_{1} \ldots \epsilon_{m} \epsilon \epsilon \ldots
$$

Since $\epsilon$ is minimal, this implies $m=0$, so $k|| \epsilon \mid$. On the other hand, if $k<0$ then $(p,-k, p)=(p, k, p)^{-1} \in$ $p\left(\mathcal{G}_{E}\right) p$ and the same argument establishes $k|| \epsilon \mid$. The conclusion is that $p\left(\mathcal{G}_{E}\right) p$ is the infinite cyclic group generated by $(p,|\epsilon|, p)$.

(2) Let $(p, k, p) \in p\left(\mathcal{G}_{E}\right) p$. Then $p \sim_{k} p$ implies $p=\alpha x=\beta x$ for some $(\alpha, \beta) \in E^{\star} \times_{r} E^{\star}$ and $x \in r(\alpha) \partial E$, with $|\alpha|-|\beta|=k$. If $p$ is finite, this implies $\alpha=\beta$, so $k=0$. That is, the isotropy group at $p$ is trivial. On the other hand, suppose $p$ is infinite and not eventually periodic. If $|\alpha|<|\beta|$, then $\beta=\alpha \beta^{\prime}$ for some $\beta^{\prime} \in E^{\star}$. But then $p=\alpha x=\beta x=\alpha \beta^{\prime} x$, so $x=\beta^{\prime} x=\beta^{\prime} \beta^{\prime} x=\beta^{\prime} \beta^{\prime} \beta^{\prime} \ldots$, and this proves $p$ is eventually periodic, a contradiction. Similarly, assuming $|\beta|<|\alpha|$ reaches the same contradiction. Therefore, $|\alpha|=|\beta|$ and $k=0$, implying that the isotropy group at $p$ is trivial.

The next step is to define a topology on $\mathcal{G}_{E}$. Let $(\alpha, \beta) \in E^{\star} \times_{r} E^{\star}$, and let $F \subseteq_{\text {finite }} r(\alpha) E^{1}$. Define the sets:

$$
\mathcal{Z}(\alpha, \beta)=\{(\alpha x,|\alpha|-|\beta|, \beta x) \mid x \in r(\alpha) \partial E\} ; \quad \mathcal{Z}(\alpha, \beta, F)=\mathcal{Z}(\alpha, \beta) \backslash \bigcup_{e \in F} \mathcal{Z}(\alpha e, \beta e) .
$$

Obviously, $\mathcal{Z}(\alpha, \beta)=\mathcal{Z}(\alpha, \beta, \emptyset)$. Next we present a pair of technical lemmas (generalising [50, Lemma $2.5])$ which prove that the collection of sets of the form $\mathcal{Z}(\alpha, \beta, F)$ is closed under pairwise intersections, so it can serve as a base for a topology on $\mathcal{G}_{E}$.

Lemma 2.13. Let $(\alpha, \beta),(\gamma, \delta) \in E^{\star} \times_{r} E^{\star}$. Then

$$
\mathcal{Z}(\alpha, \beta) \cap \mathcal{Z}(\gamma, \delta)= \begin{cases}\mathcal{Z}(\alpha, \beta) & \text { if } \exists \kappa \in E^{\star}, \alpha=\gamma \kappa, \beta=\delta \kappa \\ \mathcal{Z}(\gamma, \delta) & \text { if } \exists \kappa \in E^{\star}, \gamma=\alpha \kappa, \delta=\beta \kappa \\ \emptyset & \text { otherwise }\end{cases}
$$

Proof. We prove that when the intersection of the two sets is nonempty, then it must be one of the first two cases in the piecewise expression. To this end, let $(\alpha x,|\alpha|-|\beta|, \beta x)=\left(\gamma x^{\prime},|\gamma|-|\delta|, \delta x^{\prime}\right) \in$ $\mathcal{Z}(\alpha, \beta) \cap \mathcal{Z}(\gamma, \delta)$, where $x \in r(\alpha) \partial E$ and $x^{\prime} \in r(\gamma) \partial E$. Assume $|\gamma| \leq|\alpha|$, which implies $|\delta| \leq|\beta|$; if not, 
rearrange. Since $\alpha x=\gamma x^{\prime}$, it must be that $\alpha=\gamma \kappa$ where $\kappa$ is the initial subpath of $x^{\prime}$ of length $|\alpha|-|\gamma|$. Similarly, $\beta=\delta \kappa$. So we are in the first case (or the second case, if a rearrangement took place). In the first two cases in the piecewise expression, it is clear from the definitions what the intersection of $\mathcal{Z}(\alpha, \beta)$ and $\mathcal{Z}(\gamma, \delta)$ must be.

Lemma 2.14. Suppose $(\alpha, \beta),(\gamma, \delta) \in E^{\star} \times_{r} E^{\star}, F \subseteq_{\text {finite }} r(\alpha) E^{1}$, and $H \subseteq$ finite $r(\gamma) E^{1}$. Then

$$
\mathcal{Z}(\alpha, \beta, F) \cap \mathcal{Z}(\gamma, \delta, H)= \begin{cases}\mathcal{Z}(\alpha, \beta, F \cup H) & \text { if } \alpha=\gamma, \beta=\delta \\ \mathcal{Z}(\alpha, \beta, F) & \text { if } \exists \kappa \in E^{\star},|\kappa| \geq 1, \alpha=\gamma \kappa, \beta=\delta \kappa, \kappa_{1} \notin H \\ \mathcal{Z}(\gamma, \delta, H) & \text { if } \exists \kappa \in E^{\star},|\kappa| \geq 1, \gamma=\alpha \kappa, \delta=\beta \kappa, \kappa_{1} \notin F \\ \emptyset & \text { otherwise }\end{cases}
$$

Proof. We make a calculation and then proceed by cases:

$$
\begin{aligned}
\mathcal{Z}(\alpha, \beta, F) \cap \mathcal{Z}(\gamma, \delta, H) & =\left[\mathcal{Z}(\alpha, \beta) \backslash \bigcup_{e \in F} \mathcal{Z}(\alpha e, \beta e)\right] \cap\left[\mathcal{Z}(\gamma, \delta) \backslash \bigcup_{e \in H} \mathcal{Z}(\gamma e, \delta e)\right] \\
& =[\mathcal{Z}(\alpha, \beta) \cap \mathcal{Z}(\gamma, \delta)] \backslash\left[\bigcup_{e \in F} \mathcal{Z}(\alpha e, \beta e) \cup \bigcup_{e \in H} \mathcal{Z}(\gamma e, \delta e)\right] .
\end{aligned}
$$

Case 1: If $\alpha=\gamma$ and $\beta=\delta$, equation (2.4) yields $\mathcal{Z}(\alpha, \beta, F) \cap \mathcal{Z}(\gamma, \delta, H)=\mathcal{Z}(\alpha, \beta, F \cup H)$.

Case 2: If there exists $\kappa \in E^{\star} \backslash E^{0}$ such that $\alpha=\gamma \kappa$ and $\beta=\delta \kappa$ then after applying Lemma 2.13, the right hand side of (2.4) becomes

$$
\mathcal{Z}(\alpha, \beta) \backslash\left[\bigcup_{e \in F} \mathcal{Z}(\alpha e, \beta e) \cup \bigcup_{e \in H} \mathcal{Z}(\gamma e, \delta e)\right] .
$$

Moreover, $\mathcal{Z}(\alpha, \beta) \cap \mathcal{Z}(\gamma e, \delta e)=\emptyset$ for all $e \in H$, provided $e \neq \kappa_{1}$. If $e=\kappa_{1}$ then $\mathcal{Z}(\alpha, \beta) \cap \mathcal{Z}(\gamma e, \delta e)=$ $\mathcal{Z}(\alpha, \beta)$. Therefore $(2.4)$ becomes $\mathcal{Z}(\alpha, \beta, F)$ if $\kappa_{1} \notin H$ and $\emptyset$ if $\kappa_{1} \in H$.

Case 3: If there exists $\kappa \in E^{\star} \backslash E^{0}$ such that $\gamma=\alpha \kappa$ and $\delta=\beta \kappa$ then the situation is symmetric to the second case.

Case 4: Otherwise, $\mathcal{Z}(\alpha, \beta) \cap \mathcal{Z}(\gamma, \delta)=\emptyset$, by Lemma 2.13.

From now on, we assume $\mathcal{G}_{E}$ has the topology generated by all the sets:

$$
\mathcal{Z}(\alpha, \beta, F) ; \quad(\alpha, \beta) \in E^{\star} \times_{r} E^{\star}, F \subseteq \text { finite } r(\alpha) E^{1} .
$$

Some of our references give a different base for the topology on $\mathcal{G}_{E}$, but all the different bases that we know of contain the sets $\mathcal{Z}(\alpha, \beta, F)$. There are advantages to working with a base that is not too large, which is why we have chosen to focus on this one.

Let $E$ be a graph and consider $\mathbb{Z}$ with the discrete topology. The map

$$
\theta: \mathcal{G}_{E} \rightarrow \mathbb{Z}, \quad(x, k, y) \mapsto k,
$$

is a continuous groupoid homomorphism. In fact, it is a degree map giving $\mathcal{G}_{E}$ the structure of a $\mathbb{Z}$-graded groupoid. Some parts of this lemma are reminiscent of [50, Proposition 2.6].

Lemma 2.15. Let E be a graph.

(1) The topology on $\mathcal{G}_{E}$ is Hausdorff.

(2) $\boldsymbol{d}: \mathcal{G}_{E} \rightarrow \partial E$ is a local homeomorphism.

(3) If $(\alpha, \beta) \in E^{\star} \times_{r} E^{\star}$ and $F \subseteq$ finite $r(\alpha) E^{1}$, then $\mathcal{Z}(\alpha, \beta, F)$ is compact. 
Proof. (1) Take $(x, k, y) \neq(w, \ell, z)$ in $\mathcal{G}_{E}$. If $k \neq \ell$ then $\theta^{-1}(k)$ and $\theta^{-1}(\ell)$ are disjoint open sets separating the two points. Otherwise, either $x \neq w$ or $y \neq z$. If $w \neq x$ then either: $w$ and $x$ must differ on some initial segment, or one must be an initial subpath of the other. Using Lemma 2.14, it is not difficult to separate the two points by disjoint open sets. If $y \neq z$, the same reasoning applies.

(2) For $(\alpha, \beta) \in E^{\star} \times_{r} E^{\star}$, define

$$
h_{\alpha, \beta}: Z(\beta) \rightarrow \mathcal{Z}(\alpha, \beta), \quad \quad \beta x \mapsto(\alpha x,|\alpha|-|\beta|, \beta x) .
$$

Clearly, $h_{\alpha, \beta}$ is a bijection. By Lemma 2.14, the basic open sets contained in $\mathcal{Z}(\alpha, \beta)$ are all of the form $\mathcal{Z}\left(\alpha \kappa, \beta \kappa, F^{\prime}\right)$ where $\kappa \in r(\alpha) E^{\star}$ and $F^{\prime} \subseteq_{\text {finite }} r(\kappa) E^{1}$. Clearly

$$
h_{\alpha, \beta}^{-1}\left(\mathcal{Z}\left(\alpha \kappa, \beta \kappa, F^{\prime}\right)\right)=Z\left(\beta \kappa, F^{\prime}\right)
$$

is open in $Z(\beta)$, so $h_{\alpha, \beta}$ is continuous. A continuous map from a compact space to a Hausdorff space is a closed map, so $h_{\alpha, \beta}$ is a closed map. Therefore $h_{\alpha, \beta}$ is a homeomorphism. This proves that $\left.\boldsymbol{d}\right|_{\mathcal{Z}(\alpha, \beta)}$ is a homeomorphism onto its image (because $\left.\left.\boldsymbol{d}\right|_{\mathcal{Z}(\alpha, \beta)} ^{-1}=h_{\alpha, \beta}\right)$.

(3) According to item (2), $\boldsymbol{d}$ restricts to a homeomorphism $\mathcal{Z}(\alpha, \beta, F) \approx Z(\beta, F)$, and $Z(\beta, F)$ is compact by Theorem 2.4 .

Since $\mathcal{Z}(\alpha, \beta, F) \approx Z(\beta, F)$, Lemma 2.7 implies that $\mathcal{Z}(\alpha, \beta, F)=\emptyset$ if and only if $r(\alpha)$ is a regular vertex and $F=r(\alpha) E^{1}$.

Remark 2.16. The groupoid $\mathcal{G}_{E}$ admits continuous maps

$$
\boldsymbol{c}:(x, k, y) \mapsto x, \quad \theta:(x, k, y) \mapsto k, \quad \boldsymbol{d}:(x, k, y) \mapsto y,
$$

so it is tempting to think that the topology on $\mathcal{G}_{E}$ coincides with the relative topology that it gets from being a subset of the product space $\partial E \times \mathbb{Z} \times \partial E$. However, this is not the case: the topology on $\mathcal{G}_{E}$ is much finer than the relative topology from $\partial E \times \mathbb{Z} \times \partial E$.

The main theorem that follows is not new, and it has been in use for some time. Indeed, it is implied by [61, Lemma 2.1], although not in a trivial way (see also [57, Theorem 3.5] and [71, Theorem 3.16]). However, this is the first self-contained proof that we know of that applies to ordinary directed graphs, and does not require the graph to be countable.

Theorem 2.17. Let $E$ be a graph. The groupoid $\mathcal{G}_{E}$ is a Hausdorff ample groupoid with the base of compact open bisections given in (2.5).

Proof. The most technical part that remains is showing that the composition map $\boldsymbol{m}$ is continuous. If $x, z \in E^{\star} \cap \partial E$ are tail equivalent finite paths, then $(x,|x|-|z|, z)$ has a neighbourhood base of open sets, $\mathcal{N}_{(x,|x|-|z|, z)}=\left\{\mathcal{Z}(x, z, F) \mid F \subseteq_{\text {finite }} r(x) E^{1}\right\}$. If $x, z \in E^{\infty}$ are tail equivalent infinite paths, with lag $t$, then there exists $N \geq 0$ such that $\sigma^{N+t}(x)=\sigma^{N}(z)$. Consequently $(x, t, z)$ has a neighbourhood base of open sets, $\mathcal{N}_{(x, t, z)}=\left\{\mathcal{Z}\left(x_{1} \ldots x_{n+t}, z_{1} \ldots z_{n}\right) \mid n>N\right\}$.

Now suppose $U$ is an open set in $\mathcal{G}_{E}$ containing a product of two morphisms $(x, k+\ell, z)=(x, k, y)(y, \ell, z)$. It must be that $x, y, z$ are all finite paths or they are all infinite paths. If $x, y, z$ are finite paths,

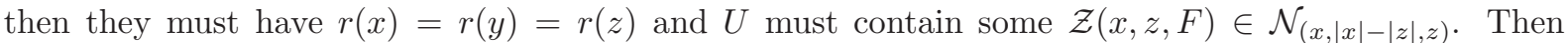
$((x, k, y),(y, \ell, z))$ is contained in the open set $(\mathcal{Z}(x, y, F) \times \mathcal{Z}(y, z, F)) \cap \mathcal{G}_{E}^{(2)}$ which is mapped bijectively by $\boldsymbol{m}$ into $Z(x, z, F) \subseteq U$. Otherwise $x, y, z$ are all infinite paths, and there must exist $n$ large enough that $\sigma^{n+k+\ell}(x)=\sigma^{n+\ell}(y)=\sigma^{n}(z)$. Making $n$ even larger if necessary, we can assume $U$ contains some $\mathcal{Z}\left(x_{1} \ldots x_{n+k+\ell}, z_{1} \ldots z_{n}\right) \in \mathcal{N}_{(x, k+\ell, z)}$. Define:

$$
x^{\prime}=x_{1} \ldots x_{n+k+\ell}, \quad y^{\prime}=y_{1} \ldots y_{n+\ell}, \quad z^{\prime}=z_{1} \ldots z_{n} .
$$

Then $((x, k, y),(y, \ell, z))$ is contained in the open set $\left(\mathcal{Z}\left(x^{\prime}, y^{\prime}\right) \times \mathcal{Z}\left(y^{\prime}, z^{\prime}\right)\right) \cap \mathcal{G}_{E}^{(2)}$, which is mapped bijectively by $\boldsymbol{m}$ into $Z\left(x^{\prime}, z^{\prime}\right) \subseteq U$. Since $(x, k+\ell, z)=(x, k, y)(y, \ell, z)$ was an arbitrary product in $U$, this shows that $\boldsymbol{m}^{-1}(U)$ is open in $\mathcal{G}_{E}^{(2)}$, so $\boldsymbol{m}$ is continuous. It is much easier to show that the inversion map $\boldsymbol{i}$ is continuous, because $\boldsymbol{i}$ puts $Z(\alpha, \beta, F)$ in bijection with $Z(\beta, \alpha, F)$. We have proved $\mathcal{G}_{E}$ is a 
topological groupoid. In Lemma 2.15 (2), it is shown that $\boldsymbol{d}$ is a local homeomorphism. Therefore, $\mathcal{G}_{E}$ is an étale groupoid. The remaining facts from Lemma 2.15 establish that $\mathcal{G}_{E}$ is a Hausdorff ample groupoid and that the base described in (2.5) consists of compact open bisections.

\section{The LeavitT Path Algebra of A GRAPH}

In $\S 3.1$, we define the Leavitt path algebra of a graph. We define it in terms of its universal property, and then describe how it can be realised as the quotient of a path algebra. Path algebras are, in some sense, the definitive examples of $\mathbb{Z}$-graded algebras, and the $\mathbb{Z}$-grading survives in their Leavitt path algebra quotients. In $\S 3.2$, we prove the Graded Uniqueness Theorem for Leavitt path algebras. In $\S 3.3$, we prove the cornerstone result: the Leavitt path algebra of a graph is isomorphic to the Steinberg algebra of its boundary path groupoid. Through this lens, we rederive some fundamentals of Leavitt path algebras, and classify finite-dimensional Leavitt path algebras. In $§ 3.4$, we prove the Graded and Cuntz-Krieger Uniqueness Theorems for Steinberg algebras and use them to prove the Cuntz-Krieger Uniqueness Theorem for Leavitt path algebras.

Remark 3.1. Historically, the theory of Leavitt path algebras was developed for the case when $R$ is a field, and $E$ is a row-finite countable graph. Later, the methods were improved and $R$ could be any unital commutative ring if $E$ is a countable graph [4, 68]. Alternatively, $E$ could be an arbitrary graph if $R$ is a field $[2,43]$. The proofs of some key results, including the fact that the relations on $L_{R}(E)$ do not collapse the algebra to zero ([43, Lemma 1.5] and [68, Proposition 3.4]) and the Graded Uniqueness Theorem ([43, Proposition 3.6] and [68, Theorem 5.3]), have not yet been recorded for the case where simultaneously $E$ is uncountable and $R$ is not a field. Here, we fix this and complete the picture.

3.1. Introducing Leavitt path algebras. Let $E=\left(E^{0}, E^{1}, r, s\right)$ be a graph. We introduce the set of formal symbols $\left(E^{1}\right)^{*}=\left\{e^{*} \mid e \in E^{1}\right\}$ and call the elements of $\left(E^{1}\right)^{*}$ ghost edges. For clarity, we will sometimes refer to the elements of $E^{1}$ as real edges. If $\alpha=\alpha_{1} \ldots \alpha_{|\alpha|} \in E^{\star}$ is a finite path of positive length, we define $\alpha^{*}$ to be the sequence $\alpha_{|\alpha|}^{*} \ldots \alpha_{1}^{*}$, and call it a ghost path. We also define $v^{*}=v$ for every $v \in E^{0}$.

Definition 3.2. [68] Let $E$ be a graph and let $A$ be a ring. Assume $\left\{v, e, e^{*} \mid v \in E^{0}, e \in E^{1}\right\}$ is a subset of $A$; in other words, there is a function $E^{0} \sqcup E^{1} \sqcup\left(E^{1}\right)^{*} \rightarrow A$ whose image inherits the notation of its domain. Then $\left\{v, e, e^{*} \mid v \in E^{0}, e \in E^{1}\right\} \subset A$ is called a Leavitt $E$-family if the following conditions are satisfied:

(V) $v^{2}=v$ and $v w=0$ for all $v, w \in E^{0}, v \neq w$;

(E1) $s(e) e=e r(e)=e$ for all $e \in E^{1}$;

(E2) $e^{*} s(e)=r(e) e^{*}=e^{*}$ for all $e \in E^{1}$;

(CK1) $e^{*} e=r(e)$ and $e^{*} f=0$ for all $e, f \in E^{1}, e \neq f$;

(CK2) $v=\sum_{e \in v E^{1}} e e^{*}$ for all $v \in E_{\mathrm{reg}}^{0}$.

The interpretation of $(\mathrm{V})$ is that $\left\{v \in A \mid v \in E^{0}\right\}$ is a set of pairwise orthogonal idempotents. The relations (CK1) and (CK2) are called the Cuntz-Krieger relations, and they originate from operator theory. The relevant interpretation, at least in that setting, is that vertices are represented by projections, and edges are represented by partial isometries with mutually orthogonal ranges.

In any algebra $A$ containing a Leavitt $E$-family $\left\{v, e, e^{*} \mid v \in E^{0}, e \in E^{1}\right\}$, one can consider paths $\mu=\mu_{1} \ldots \mu_{|\mu|}$ and ghost paths $\mu^{*}=\mu_{|\mu|}^{*} \ldots \mu_{1}^{*}$ as elements of $A$ in the obvious way: products of their constituent real edges and ghost edges respectively. The following lemma is straightforward to prove using the relations (E1), (E2), and (CK1). It is so fundamental that we will usually use the result without referring to it. 
Lemma 3.3. If $A$ is an R-algebra generated by a Leavitt E-family $\left\{v, e, e^{*} \mid v \in E^{0}, e \in E^{1}\right\}$, the elements of $A$ obey the rule:

$$
\left(r \mu \nu^{*}\right)\left(r^{\prime} \gamma \lambda^{*}\right)= \begin{cases}\left(r r^{\prime}\right) \mu \kappa^{*} \lambda^{*} & \text { if } \gamma \text { is an initial subpath of } \nu, \text { with } \nu=\gamma \kappa \\ \left(r r^{\prime}\right) \mu \kappa \lambda^{*} & \text { if } \nu \text { is an initial subpath of } \gamma, \text { with } \gamma=\nu \kappa \\ 0 & \text { otherwise }\end{cases}
$$

for all $r, r^{\prime} \in R$ and all $\mu, \nu, \gamma, \lambda \in E^{\star}$, with $r(\mu)=r(\nu)$ and $r(\gamma)=r(\lambda)$.

Corollary 3.4. Every R-algebra generated by a Leavitt E-family is generated, as an abelian group, by the set $\left\{r \alpha \beta^{*} \mid r \in R,(\alpha, \beta) \in E^{\star} \times{ }_{r} E^{\star}\right\}$.

Proof. By Lemma 3.3, every word in the generators $\left\{v, e, e^{*} \mid v \in E^{0}, e \in E^{1}\right\}$ reduces to an expression of the form $\alpha \beta^{*}$ where $\alpha, \beta \in E^{\star}$. Moreover, $\alpha \beta^{*}=0$ unless $r(\alpha)=r(\beta)$, by (V), (E1), and (E2).

Let $B$ be an $R$-algebra generated by a Leavitt $E$-family $\left\{v, e, e^{*} \mid v \in E^{0}, e \in E^{1}\right\}$. We say that $B$ is universal (for Leavitt $E$-families) if every $R$-algebra $A$ containing a Leavitt $E$-family $\left\{a_{v}, b_{e}, c_{e^{*}} \mid v \in\right.$ $\left.E^{0}, e \in E^{1}\right\}$ admits a unique $R$-algebra homomorphism $\pi: B \rightarrow A$ such that $\pi(v)=a_{v}, \pi(e)=b_{e}$, and $\pi\left(e^{*}\right)=c_{e^{*}}$ for every $v \in E^{0}$ and $e \in E^{1}$. The universal property determines $B$ up to isomorphism.

Definition 3.5. Let $E$ be a graph. The Leavitt path algebra of $E$ with coefficients in $R$, denoted by $L_{R}(E)$, is the universal $R$-algebra generated by a Leavitt $E$-family.

Technically, $L_{R}(E)$ is an isomorphism class in the category of $R$-algebras. If $B$ is a specific $R$-algebra having the universal property for Leavitt $E$-families, then $B$ is a model of $L_{R}(E)$. However, it is customary and natural to refer to $L_{R}(E)$ as if it were a specific model with the standard generators $\left\{v, e, e^{*} \mid v \in\right.$ $\left.E^{0}, e \in E^{1}\right\}$. Every element $x \in L_{R}(E)$, so to speak, is a finite sum of the form $x=\sum r_{i} \alpha_{i} \beta_{i}^{*}$ where $r_{i} \in R$ and $(\alpha, \beta) \in E^{\star} \times{ }_{r} E^{\star}$ for all $i$. Such an expression for $x$ is not necessarily unique, owing to the (CK2) relation. If we have reason to consider a different model of $L_{R}(E)$, say another $R$-algebra $B$, then we would write $L_{R}(E) \cong B$.

Examples 3.6. [2, $\S 1.3]$ Sometimes $L_{R}(E)$ can be recognised as a more familiar algebra. Four fundamental examples of Leavitt path algebras are:

(a) The finite line graph with $n$ vertices is the graph pictured below:

$$
A_{n}=\quad v^{v_{1}} \stackrel{e_{1}}{\longrightarrow} \bullet^{v_{2}} \stackrel{e_{2}}{\longrightarrow} \bullet v^{v_{3}} \ldots \ldots \ldots \ldots . . . . \bullet^{v_{n-1}} \stackrel{e_{n-1}}{\longrightarrow} \bullet v_{n}
$$

It turns out that $L_{R}\left(A_{n}\right) \cong M_{n}(R)$, the matrix algebra of $n \times n$ matrices over $R$. Explicitly, the set of standard matrix units $\left\{E_{i, j} \mid 1 \leq i, j \leq n\right\} \subset M_{n}(R)$ contains a Leavitt $E$-family $\left\{a_{v}, b_{e}, c_{e^{*}} \mid\right.$ $\left.v \in A_{n}^{0}, e \in A_{n}^{1}\right\}$, where:

$$
a_{v_{i}}=E_{i, i}, \quad b_{e_{j}}=E_{j, j+1}, \quad c_{e_{j}^{*}}=E_{j+1, j}, \quad 1 \leq i \leq n, 1 \leq j \leq n-1 .
$$

(b) The rose with $n$ petals is the graph pictured below (see also Example 2.11):

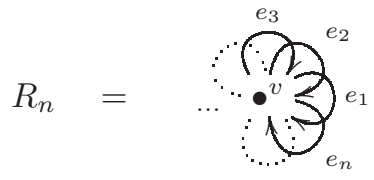

The Leavitt path algebra $L_{R}\left(R_{n}\right)$ is isomorphic to the Leavitt algebra $L_{n, R}$, discovered by W. G. Leavitt in $[51, \S 3]$. It is from this example that the Leavitt path algebras get their name.

(c) The rose with 1 petal,

$$
R_{1}=v^{v} \bullet e
$$

gives rise to the algebra of Laurent polynomials $R\left[x, x^{-1}\right]$. 
(d) The Toeplitz graph,

$$
T=e \mathrm{C}^{u} \bullet \stackrel{f}{\longrightarrow} \bullet v
$$

gives rise to the Toeplitz $R$-algebra, which has the presentation $R\langle x, y \mid x y=1\rangle$. The isomorphism $R\langle x, y \mid x y=1\rangle \rightarrow L_{K}(T)$ maps $x \mapsto e^{*}+f^{*}$ and $y \mapsto e+f$.

As an alternative to Definition 3.5, it is popular to define the Leavitt path algebra of a graph as a certain quotient of a path algebra. The path algebra of a graph (also called the quiver algebra of a quiver) is an older concept, familiar to a wider audience of algebraists and representation theorists. We have defined $L_{R}(E)$ by its universal property, so we look towards path algebras to provide a model of $L_{R}(E)$, thereby proving that $L_{R}(E)$ exists.

Let $E=\left(E^{0}, E^{1}, r, s\right)$ be a graph. The path algebra of $E$ with coefficients in $R$ is the free $R$-algebra generated by $E^{0} \sqcup E^{1}$, modulo the ideal generated by the relations (V) and (E1). The extended graph of $E$ is defined as $\widehat{E}=\left(E^{0}, E^{1} \sqcup\left(E^{1}\right)^{*}, r^{\prime}, s^{\prime}\right)$, where $r^{\prime}$ and $s^{\prime}$ are extensions of $r$ and $s$, respectively:

$$
\begin{array}{ll}
r^{\prime}(e)=r(e) \text { for all } e \in E^{1} ; & r^{\prime}\left(e^{*}\right)=s(e) \text { for all } e^{*} \in\left(E^{1}\right)^{*} \\
s^{\prime}(e)=s(e) \text { for all } e \in E^{1} ; & s^{\prime}\left(e^{*}\right)=r(e) \text { for all } e^{*} \in\left(E^{1}\right)^{*} .
\end{array}
$$

In other words, $\widehat{E}$ is formed from $E$ by adding a new edge $e^{*}$ for each edge $e$, such that $e^{*}$ has the opposite direction to $e$. The path algebra $R \widehat{E}$ can be characterised as the free $R$-algebra generated by $E^{0} \sqcup E^{1} \sqcup\left(E^{1}\right)^{*}$, subject to the relations $(\mathrm{V}),(\mathrm{E} 1)$, and $(\mathrm{E} 2)$. Let $\mathcal{A}$ be the quotient of $R \widehat{E}$ by the ideal generated by the relations (CK1) and (CK2). By virtue of its construction, $\mathcal{A}$ has the universal property for Leavitt $E$-families, and consequently $\mathcal{A} \cong L_{R}(E)$. The path algebra model is useful for proving the following fact.

Proposition 3.7. The Leavitt path algebra $L_{R}(E)=\bigoplus_{n \in \mathbb{Z}} L_{R}(E)_{n}$ is a $\mathbb{Z}$-graded algebra, where the homogeneous components are:

$$
L_{R}(E)_{n}=\operatorname{span}_{R}\left\{\mu \nu^{*}\left|(\mu, \nu) \in E^{\star} \times_{r} E^{\star},\right| \mu|-| \nu \mid=n\right\} .
$$

Proof. Naturally, the free $R$-algebra $R\left\langle E^{0} \cup E^{1} \cup\left(E^{1}\right)^{*}\right\rangle$ is $\mathbb{Z}$-graded by setting $\operatorname{deg}(v)=0$ for all $v \in E^{0}$, and $\operatorname{deg}(e)=1, \operatorname{deg}\left(e^{*}\right)=-1$ for all $e \in E^{1}$. Extending the degree map (in the only possible way) yields $\operatorname{deg}\left(a_{1} \ldots a_{n}\right)=\sum_{i=1}^{n} \operatorname{deg}\left(a_{n}\right)$ for any word $a_{1} \ldots a_{n} \in R\left\langle E^{0} \cup E^{1} \cup\left(E^{1}\right)^{*}\right\rangle$. The relations (V), (E1), and (E2) are all homogeneous with respect to the grading on $R\left\langle E^{0} \cup E^{1} \cup\left(E^{1}\right)^{*}\right\rangle$, so they generate a graded ideal, and the quotient $R \widehat{E}$ is $\mathbb{Z}$-graded. Similarly, relations (CK1) and (CK2) are homogeneous with respect to the grading on $R \widehat{E}$, so they generate a graded ideal, and the quotient $L_{R}(E)$ is $\mathbb{Z}$-graded. The word $\mu \nu^{*}$ has degree $|\mu|-|\nu|$ in $R\left\langle E^{0} \cup E^{1} \cup\left(E^{1}\right)^{*}\right\rangle$, which gives the expression for the homogeneous components of $L_{R}(E)$.

3.2. Uniqueness theorems for Leavitt path algebras. Research on graph algebras has made extensive use of two main kinds of uniqueness theorems: the Cuntz-Krieger uniqueness theorems, and the graded uniqueness theorems. (In the analytic setting, graded uniqueness theorems are replaced by gauge invariant uniqueness theorems.) These theorems give sufficient conditions for a homomorphism to be injective, so they are very useful for establishing isomorphisms between a graph algebra and another algebra that comes from somewhere else. They are also very useful for studying structural properties like primeness and simplicity. Appropriate versions of these theorems have been proved not just for Leavitt path algebras but also (and we refer to $[30,31,58,61]$ ) for graph $C^{*}$-algebras, as well as Cohn path algebras, higher-rank graph algebras, and even algebras of topological higher-rank graphs.

This section provides a brief account of the uniqueness theorems for Leavitt path algebras. For the Graded Uniqueness Theorem, we adhere to Tomforde's proof from [68].

Lemma 3.8. [68, Lemma 5.1] Let I be a graded ideal of $L_{R}(E)$, where $E$ is a graph. Then $I$ is generated as an ideal by its 0 -component $I_{0}=I \cap L_{R}(E)_{0}$. 
Proof. Since $I$ is a graded ideal, $I=\sum_{k \in \mathbb{Z}} I_{k}$, where $I_{k}=I \cap L_{R}(E)_{k}$. Let $k>0$ and $x \in I_{k}$. By Corollary 3.4, we can write $x=\sum_{i=1}^{n} \alpha_{i} x_{i}$ where each $x_{i} \in L_{R}(E)_{0}$, and each $\alpha_{i} \in E^{\star}$ is distinct with $\left|\alpha_{i}\right|=k$. Then for $1 \leq j \leq n$, we have $x_{j}=\alpha_{j}^{*}\left(\sum_{i=1}^{n} \alpha_{i} x_{i}\right)=\alpha_{j}^{*} x \in I_{0}$. So, $I_{k}$ is spanned by elements of the form $\alpha_{j} x_{j}$ where $\alpha_{j} \in L_{R}(E)_{k}$ and $x_{j} \in I_{0}$. That is, $I_{k}=L_{R}(E)_{k} I_{0}$. Similarly, if $y \in I_{-k}$ then we can write $y=\sum_{i=1}^{m} y_{i} \beta_{i}^{*}$ where each $y_{i} \in L_{R}(E)_{0}$, and each $\beta_{i} \in E^{\star}$ is distinct with $\left|\beta_{i}\right|=k$. Then for $1 \leq j \leq n$, we have $y_{j}=\left(\sum_{i=1}^{n} y_{i} \beta_{i}^{*}\right) \beta_{j}=y \beta_{j} \in I_{0}$. Therefore $I_{-k}$, is spanned by elements of the form $y_{j} \beta_{j}$ where $\beta_{j} \in L_{R}(E)_{-k}$ and $y_{j} \in I_{0}$. That is, $I_{-k}=I_{0} L_{R}(E)_{-k}$. Since $I=\sum_{n \in \mathbb{Z}} I_{k}$, this shows $I$ is the ideal generated by $I_{0}$.

The next lemma is a slight variation of the Reduction Theorem [2, Theorem 2.2.11]. The lemma needs the assumption that $r v \in L_{R}(E)$ is nonzero for every $r \in R \backslash\{0\}$ and $v \in E^{0}$. In fact, this is always true, but we shall only prove it later.

Lemma 3.9. [68, Lemma 5.2]. Let $E$ be an arbitrary graph. Assume $r v \in L_{R}(E)$ is nonzero for every $r \in R \backslash\{0\}$ and $v \in E^{0}$. If $x \in L_{R}(E)_{0}$ is nonzero, then there exists $(\alpha, \beta) \in E^{\star} \times_{r} E^{\star}$ and $s \in R \backslash\{0\}$, such that $\alpha^{*} x \beta=\operatorname{sr}(\alpha)$.

Proof. The set $\mathcal{M}_{n}=\operatorname{span}_{R}\left\{\alpha \beta^{*}|1 \leq| \alpha|=| \beta \mid \leq n\right\}$ is an $R$-submodule of $L_{R}(E)_{0}$, and indeed $L_{R}(E)_{0}=\bigcup_{n=0}^{\infty} \mathcal{M}_{n}$. The strategy is to prove inductively that for all $n \geq 0$ the claim holds: for all $0 \neq x \in \mathcal{M}_{n}$ there exists $(\alpha, \beta) \in E^{\star} \times_{r} E^{\star}$ and $s \in R \backslash\{0\}$ such that $\alpha^{*} x \beta=\operatorname{sr}(\alpha)$. The base case is $n=0$. If $x \in \mathcal{M}_{0}$ then $x$ is a linear combination of vertices. Say $x=\sum_{i} r_{i} v_{i}$ with the $v_{i}$ being distinct vertices and the $r_{i} \in R \backslash\{0\}$. Then $v_{1} x v_{1}=r_{1} v_{1}$ proves the claim. Now assume the claim holds for $n-1$. Let $0 \neq x \in \mathcal{M}_{n}$. We can write

$$
x=\sum_{i=1}^{p} r_{i} \alpha_{i} \beta_{i}^{*}+\sum_{j=1}^{q} s_{j} v_{j}
$$

where for all $1 \leq i \leq p$ and all $1 \leq j \leq q: r_{i}, s_{j} \in R \backslash\{0\},\left(\alpha_{i}, \beta_{i}\right) \in E^{\star} \times_{r} E^{\star}$ with $1 \leq\left|\alpha_{i}\right|=\left|\beta_{i}\right| \leq n$, and $v_{j} \in E^{0}$. Further assume that all the $\left(\alpha_{i}, \beta_{i}\right)$ are distinct and all the $v_{j}$ are distinct. In the first case, if $v_{j}$ is a sink for some $1 \leq j \leq q$, then $v_{j} x v_{j}=s_{j} v_{j}$ proves the claim. In the second case, if $v_{j}$ is an infinite emitter for some $1 \leq j \leq q$, then there is an edge $e \in v_{j} E^{1} \backslash\left\{\left(\alpha_{1}\right)_{1}, \ldots,\left(\alpha_{p}\right)_{1}\right\}$ and $e^{*} x e=s_{j} r(e)$ proves the claim. Otherwise, in the third case, every $v_{j}$ is a regular vertex. Applying (CK2), it is possible to expand $v_{j}=\sum_{e \in v_{j} E^{1}} e e^{*}$ for all $1 \leq j \leq q$. Then (3.1) can be rewritten as

$$
x=\sum_{i=1}^{p} t_{i} e_{i} \mu_{i} \nu_{i}^{*} f_{i}^{*}
$$

where $t_{i} \in R \backslash\{0\}, e_{i}, f_{i} \in E^{1}$, and $\left(e_{i} \mu_{i}, f_{i} \nu_{i}\right) \in E^{\star} \times_{r} E^{\star}$ for all $i \leq 1 \leq p$. It is safe to assume that

$$
\sum_{\substack{1 \leq j \leq p \\ e_{j}=e_{1}, f_{j}=f_{1}}} t_{j} e_{j} \mu_{j} \nu_{j}^{*} f_{j}^{*}=e_{1}\left(\sum_{\substack{1 \leq j \leq p \\ e_{j}=e_{1}, f_{j}=f_{1}}} t_{j} \mu_{j} \nu_{j}^{*}\right) f_{1}^{*} \neq 0,
$$

otherwise it could just be removed from the sum in (3.2). Then, define

$$
x^{\prime}=\sum_{\substack{1 \leq j \leq p \\ 1 \leq e_{1}, f_{j}=f_{1}}} t_{j} \mu_{j} \nu_{j}^{*},
$$

noting that $0 \neq x^{\prime} \in \mathcal{M}_{n-1}$. By the inductive assumption there exists $(\alpha, \beta) \in E^{\star} \times_{r} E^{\star}$ and $s \in R \backslash\{0\}$ such that $\alpha^{*} x^{\prime} \beta=\operatorname{sr}(\alpha)$. Clearly $x^{\prime}=e_{1}^{*} x f_{1}$, so $\alpha^{*} x^{\prime} \beta=\alpha^{*} e_{1}^{*} x f_{1} \beta=\operatorname{sr}(\alpha)$. By assumption, $\operatorname{sr}(\alpha) \neq 0$; this implies $e_{1} \alpha$ and $f_{1} \beta$ are legitimate paths with the same range. The claim is now proved for $n$, and by mathematical induction it holds for all $n \geq 0$.

Combining these lemmas proves the Graded Uniqueness Theorem for Leavitt path algebras. This generalises both [68, Theorem 5.3] and [43, Theorem 3.2] by removing any restrictions on the cardinality of $E$, and by not requiring $R$ to be a field. However, we emphasise that this is essentially Tomforde's proof with the insight that countability is not required. 
Theorem 3.10 (Graded Uniqueness Theorem for Leavitt path algebras).

Let $E$ be a graph, and $R$ a unital commutative ring. If $A$ is a $\mathbb{Z}$-graded ring and $\pi: L_{R}(E) \rightarrow A$ is a graded homomorphism with the property that $\pi(r v) \neq 0$ for every $v \in E^{0}$ and every $r \in R \backslash\{0\}$, then $\pi$ is injective.

Proof. The first observation is that $r v \neq 0$ (because $\pi(r v) \neq 0$ ) for every $v \in E^{0}$ and $r \in R \backslash\{0\}$. The second observation is that $\operatorname{ker} \pi$ is a graded ideal, because $\pi$ is a graded homomorphism. Suppose $x \in(\operatorname{ker} \pi)_{0}=\operatorname{ker} \pi \cap L_{R}(E)_{0}$. If $x \neq 0$, then by Lemma 3.9, there exists $(\alpha, \beta) \in E^{\star} \times_{r} E^{\star}$ and $s \in R \backslash\{0\}$, such that $\alpha^{*} x \beta=\operatorname{sr}(\alpha)$. Then $\pi(\operatorname{sr}(\alpha))=\pi\left(\alpha^{*}\right) \pi(x) \pi(\beta)=0$, which is a contradiction. Therefore $x=0$, so $(\operatorname{ker} \pi)_{0}=0$. Lemma 3.8 proves that $\operatorname{ker} \pi$ is generated as an ideal by $(\operatorname{ker} \pi)_{0}=0$; consequently, $\operatorname{ker} \pi=0$, so $\pi$ is injective.

Corollary 3.11. For every nonzero graded ideal $I$ of $L_{R}(E)$, there exists $r \in R \backslash\{0\}$ and $v \in E^{0}$ such that $r v \in I$.

In fact, all of the uniqueness theorems have a corollary of this sort. We will not always write it so explicitly. The Cuntz-Krieger Uniqueness Theorem is similar in spirit to the Graded Uniqueness Theorem. We do not require the homomorphism to be graded, this time, but pay the price of an extra condition on the graph, called Condition (L).

Definition 3.12. A graph $E$ satisfies Condition (L) if every cycle has an exit.

Note that $E$ satisfies Condition (L) if and only if every closed path has an exit; this is fairly intuitive and it is proved in [3, Lemma 2.5]. Combining [68, Theorem 6.5] and [43, Theorem 3.6] (see also [2, Theorem 2.2.16]) produces a version of the Cuntz-Krieger Uniqueness Theorem for Leavitt path algebras.

Theorem 3.13. Let $E$ be a graph satisfying Condition $(L)$ and let $R$ be a unital commutative ring, such that either $E$ is countable or $R$ is a field. If $A$ is a ring and $\psi: L_{R}(E) \rightarrow A$ is a homomorphism with the property that $\psi(r v) \neq 0$ for every $v \in E^{0}$ and every $r \in R \backslash\{0\}$, then $\psi$ is injective.

This theorem can be proved for a field $R=\mathbb{K}$, using the Reduction Theorem [2, Theorem 2.2.11]. However, we shall prove it later using groupoid methods instead. In doing so, we remove the awkward restrictions on $E$ and $R$.

3.3. The Steinberg algebra model. Here, we prove the existence of a Steinberg algebra model for Leavitt path algebras, and use it to prove some fundamental facts.

Theorem 3.14. [32] Let $E$ be a graph and $R$ a unital commutative ring. Then $L_{R}(E)$ and $A_{R}\left(\mathcal{G}_{E}\right)$ are isomorphic as $\mathbb{Z}$-graded $R$-algebras.

Proof. For $v \in E^{0}$ and $e \in E^{1}$, define

$$
a_{v}=\mathbf{1}_{Z(v)}, \quad b_{e}=\mathbf{1}_{\mathcal{Z}(e, r(e))}, \quad b_{e^{*}}=\mathbf{1}_{\mathcal{Z}(r(e), e)} .
$$

We can routinely validate that $\left\{a_{v}, b_{e}, b_{e}^{*} \mid v \in E^{0}, e \in E^{1}\right\}$ is a Leavitt $E$-family. For all $e, f \in E^{1}$, $v, w \in E^{0}$, and $u \in E_{\mathrm{reg}}^{0}$ :

$$
\begin{gathered}
a_{v} a_{w}=\mathbf{1}_{Z(v)} \mathbf{1}_{Z(w)}=\mathbf{1}_{Z(v) \cap Z(w)}=\delta_{v, w} \mathbf{1}_{Z(v)}, \\
a_{s(e)} b_{e} a_{r(e)}=\mathbf{1}_{Z(s(e)) \mathcal{Z}(e, r(e)) Z(r(e))}=\mathbf{1}_{\mathcal{Z}(e, r(e))}=b_{e}, \\
a_{r(e)} b_{e^{*}} a_{s(e)}=\mathbf{1}_{Z(r(e)) \mathcal{Z}(r(e), e) Z(s(e))}=\mathbf{1}_{\mathcal{Z}(r(e), e)}=b_{e^{*}}, \\
b_{e^{*}} b_{f}=\mathbf{1}_{\mathcal{Z}(r(e), e) \mathcal{Z}(f, r(f))}=\delta_{e, f} \mathbf{1}_{Z(r(e))}=\delta_{e, f} a_{r(e)}, \\
\mathbf{1}_{Z(u)}=\mathbf{1}_{\sqcup_{e \in u E^{1}} Z(e)}=\sum_{e \in u E^{1}} \mathbf{1}_{Z(e)}=\sum_{e \in u E^{1}} b_{e} b_{e^{*}}
\end{gathered}
$$


By the universal property of Leavitt path algebras, there is a unique homomorphism of $R$-algebras $\pi: L_{R}(E) \rightarrow A_{R}\left(\mathcal{G}_{E}\right)$ such that

$$
\pi(v)=a_{v}, \quad \pi(e)=b_{e}, \quad \pi\left(e^{*}\right)=b_{e^{*}},
$$

for all $v \in E^{0}$ and $e \in E^{1}$. Evidently $\pi$ is a graded homomorphism. The Graded Uniqueness Theorem for Leavitt path algebras implies $\pi$ is injective. For a path $\mu \in E^{\star}$, if we define $b_{\mu}=b_{\mu_{1}} \ldots b_{\mu_{|\mu|}}$ and $b_{\mu^{*}}=b_{\mu_{|\mu|}^{*}} \ldots b_{\mu_{1}^{*}}$ then it turns out that $b_{\mu}=\mathbf{1}_{\mathcal{Z}(\mu, r(\mu))}$ and $b_{\mu^{*}}=\mathbf{1}_{\mathcal{Z}(r(\mu), \mu)}$. Moreover, if $\nu \in E^{\star}$ is another path with $r(\mu)=r(\nu)$, then $b_{\mu} b_{\nu}^{*}=\mathbf{1}_{\mathcal{Z}(\mu, \nu)}$. If $F \subseteq$ finite $r(\mu) E^{1}$, this yields

$$
\mathbf{1}_{\mathcal{Z}(\mu, \nu, F)}=\mathbf{1}_{\mathcal{Z}(\mu, \nu)}-\sum_{e \in F} \mathbf{1}_{\mathcal{Z}(\mu e, \nu e)}=b_{\mu} b_{\nu^{*}}-\sum_{e \in F} b_{\mu e} b_{e^{*} \nu^{*}}=\pi\left(\mu \nu^{*}-\sum_{e \in F} \mu e e^{*} \nu^{*}\right) .
$$

Therefore, $\mathbf{1}_{\mathcal{Z}(\mu, \nu, F)}$ is in the image of $\pi$. Corollary 1.14 implies that $A_{R}(\mathcal{G})$ is generated by functions of the form (3.3). We conclude that $\pi$ is surjective. Therefore, $\pi$ is an isomorphism.

In the following, we generalise [68, Propositions $3.4 \& 4.9$ ] and [43, Lemmas $1.5 \& 1.6]$ by removing restrictions on the graph and the base ring.

Corollary 3.15. Let $E$ be a graph and $R$ a unital commutative ring. Then

(1) $L_{R}(E)$ has homogeneous local units, and it has a unit if and only if $E^{0}$ is finite;

(2) The set $\left\{\mu, \mu^{*} \in L_{R}(E) \mid \mu \in E^{\star}\right\}$ is R-linearly independent in $L_{R}(E)$;

(3) For every $v \in E^{0}$ and $r \in R \backslash\{0\}, r v \neq 0$.

(4) If $r \mapsto \bar{r}$ is an involution on $R$, then there exists a unique involution $L_{R}(E) \rightarrow L_{R}(E)$ such that $r \mu \nu^{*} \mapsto \bar{r} \nu \mu^{*}$ for every $r \in R$ and $(\mu, \nu) \in E^{\star} \times_{r} E^{\star}$.

Proof. (1) From Proposition 1.20, $L_{R}(E)$ has homogeneous local units, and it has a unit if and only if $\partial E$ is compact. Since $\partial E=\bigsqcup_{v \in E^{0}} Z(v)$, and each $Z(v)$ is compact and open, it is clear that $\partial E$ is compact if and only if $E^{0}$ is finite.

(2) Since $L_{R}(E)=\bigoplus_{n \in \mathbb{Z}} L_{R}(E)_{n}$, it suffices to show that $\left\{\mu\left|\mu \in E^{\star},\right| \mu \mid=n\right\}$ and $\left\{\mu^{*}\left|\mu \in E^{\star},\right| \mu \mid=n\right\}$ are linearly independent in $L_{R}(E)$, for every $n \in \mathbb{Z}$. Equivalently, $\left\{\mathbf{1}_{\mathcal{Z}(\mu, r(\mu))}\left|\mu \in E^{\star},\right| \mu \mid=n\right\}$ and $\left\{\mathbf{1}_{\mathcal{Z}(r(\mu), \mu)}\left|\mu \in E^{\star},\right| \mu \mid=n\right\}$ are linearly independent in $A_{R}\left(\mathcal{G}_{E}\right)$, for every $n \in \mathbb{Z}$. This is clearly true, since $\mathcal{Z}(\mu, r(\mu)), \mathcal{Z}(\nu, r(\nu)) \neq \emptyset$ and $\mathcal{Z}(\mu, r(\mu)) \cap \mathcal{Z}(\nu, r(\nu))=\emptyset$ for every $\mu, \nu \in E^{\star}$ such that $\mu \neq \nu$ and $|\mu|=|\nu|$.

(3) This follows directly from (2), or just the fact that $Z(v) \neq \emptyset$ for all $v \in E^{0}$.

(4) The existence follows from Proposition 1.22. The uniqueness follows from the universal property of $L_{R}(E)$.

Item (3) in Corollary 3.15 is entirely disarmed by the Steinberg algebra model. It was noticed in the early years of Leavitt path algebras that a nontrivial proof was needed for Corollary 3.15 (3). The first proofs were written, separately, by Goodearl [43] and Tomforde [68] and they involved a representation of $L_{R}(E)$ on a free $R$-module of infinite rank $\aleph \geq \operatorname{card}\left(E^{0} \sqcup E^{1}\right)$. Here is another result from the early years of Leavitt path algebras.

Proposition 3.16. [5, Proposition 3.5] If $E$ is a graph and $\mathbb{K}$ a field, then $L_{\mathbb{K}}(E)$ is finite-dimensional if and only if $E$ is acyclic and $E^{0} \cup E^{1}$ is finite. In this case, if $v_{1}, \ldots, v_{t}$ are the sinks and $n\left(v_{i}\right)=\mid\{\alpha \in$ $\left.E^{\star} \mid r(\alpha)=v_{i}\right\} \mid$, then

$$
L_{\mathbb{K}}(E) \cong \bigoplus_{i=1}^{t} M_{n\left(v_{i}\right)}(\mathbb{K})
$$


Proof. From Proposition 1.32 we have that $L_{\mathbb{K}}(E)$ is finite-dimensional if and only if $\mathcal{G}_{E}$ is finite and discrete. If $E$ had a cycle $c$, then the isotropy group based at $c c c \ldots \in \partial E$ would be infinite. If either $E^{0}$ or $E^{1}$ were infinite, then $\partial E$ would be infinite, because $\partial E=\bigsqcup_{v \in E^{0}} Z(v)=E_{\text {sing }}^{0} \sqcup\left(\bigsqcup_{e \in E^{1}} Z(e)\right)$. Thus, $\mathcal{G}_{E}$ is finite only if $E$ is acyclic and $E^{0} \cup E^{1}$ is finite. Conversely, if $E$ is acyclic and $E^{0} \cup E^{1}$ is finite, then there are no infinite paths, and only finitely many finite paths, so $\mathcal{G}_{E}$ is finite and discrete. To prove the final sentence, note that there are $t$ orbits of sizes $n\left(v_{1}\right), \ldots, n\left(v_{t}\right)$, all with trivial isotropy groups. The structure of $L_{\mathbb{K}}(E)$ is now apparent from Proposition 1.32.

3.4. Uniqueness theorems for Steinberg algebras. Steinberg algebras also support a Cuntz-Krieger Uniqueness Theorem and a Graded Uniqueness Theorem. These were first investigated in [19] and later improved in [24] and [64]. One can think of the Cuntz-Krieger Uniqueness Theorems as saying that a certain property of a graph, namely Condition (L), or a certain property of an ample groupoid, namely effectiveness, forces a homomorphism to be injective - provided it does not annihilate any scalar multiples of a local unit. This is interesting as a first example of how a Leavitt path algebra theorem translates into the more general setting of Steinberg algebras.

Briefly, this is the order of events in this section. First, we prove the Graded Uniqueness Theorem for Steinberg algebras of graded ample groupoids. Any groupoid can be graded by the trivial group, and this simple trick obtains the Cuntz-Krieger Uniqueness Theorem for Steinberg algebras. We then use the Cuntz-Krieger Uniqueness Theorem for Steinberg algebras to prove the Cuntz-Krieger Uniqueness Theorem for Leavitt path algebras.

Definitions 3.17. An étale groupoid is

(1) effective if $\operatorname{Iso}(\mathcal{G})^{\circ}=\mathcal{G}^{(0)}$, where ${ }^{\circ}$ denotes the interior in $\mathcal{G}$;

(2) topologically principal if $\left\{x \in \mathcal{G}^{(0)} \mid x \mathcal{G} x=\{x\}\right\}$ is dense in $\mathcal{G}^{(0)}$.

Recall that a groupoid is called principal if the isotropy group at every unit is trivial. Being topologically principal amounts to having a dense set of units with trivial isotropy groups. Obviously, principal implies topologically principal. Effective does not imply topologically principal, with counterexamples in [19, Examples 6.3 and 6.4], and topologically principal does not imply effective, with counterexamples in [26, §5.1]. For a deeper understanding of effective groupoids, the upcoming lemma is essential. We state and prove the lemma for more general groupoids than just ample groupoids, mainly because there was an error in its original proof and this is an opportunity to correct it.

First, some topological comments are needed. Sets with compact closure are called precompact. A locally compact, Hausdorff étale groupoid $\mathcal{G}$ need not have a base of compact open bisections, but it does have a base of precompact open bisections [19]. Indeed, $\mathcal{G}$ has a base of open bisections. Since it is locally compact and Hausdorff, $\mathcal{G}$ has a base of open bisections, each of which is contained in a (necessarily closed) compact set, and thus has compact closure.

Lemma 3.18. [19, Lemma 3.1] Let $\mathcal{G}$ be a locally compact Hausdorff étale groupoid. Then the following are equivalent:

(1) $\operatorname{Iso}(\mathcal{G}) \backslash \mathcal{G}^{(0)}$ has empty interior in $\mathcal{G}$;

(2) $\mathcal{G}$ is effective;

(3) Every nonempty open bisection $B \subseteq \mathcal{G} \backslash \mathcal{G}^{(0)}$ contains a morphism $g \notin \operatorname{Iso}(\mathcal{G})$;

(4) For every compact set $K \subseteq \mathcal{G} \backslash \mathcal{G}^{(0)}$ and every nonempty open $U \subseteq \mathcal{G}^{(0)}$, there exists an open subset $V \subseteq U$ such that $V K V=\emptyset$.

Proof. (1) $\Rightarrow(2)$ Since $\mathcal{G}$ is étale and Hausdorff, $\mathcal{G}^{(0)}$ is clopen in $\mathcal{G}$, so $\mathcal{G}^{(0)} \subseteq$ Iso $(\mathcal{G})^{\circ}$. Now assume $\left(\operatorname{Iso}(\mathcal{G}) \backslash \mathcal{G}^{(0)}\right)^{\circ}=\emptyset$. If $S \subseteq \operatorname{Iso}(\mathcal{G})$ is open, then $S$ is a disjoint union of two open sets: $S \cap \mathcal{G}^{(0)}$ and 
$S \cap\left(\mathcal{G} \backslash \mathcal{G}^{(0)}\right)$. But $S \cap\left(\mathcal{G} \backslash \mathcal{G}^{(0)}\right) \subseteq\left(\operatorname{Iso}(\mathcal{G}) \backslash \mathcal{G}^{(0)}\right)^{\circ}=\emptyset$, so $S \subseteq \mathcal{G}^{(0)}$. This shows $\operatorname{Iso}(\mathcal{G})=\mathcal{G}^{(0)}$, which means $\mathcal{G}$ is effective.

$(2) \Rightarrow(3)$ Suppose $\mathcal{G}$ is effective. If $B \subseteq \mathcal{G} \backslash \mathcal{G}^{(0)}$ is an open bisection, then $B \subseteq \operatorname{Iso}(\mathcal{G})$ implies $B \subseteq \operatorname{Iso}(\mathcal{G})^{\circ}=\mathcal{G}^{(0)}$ and therefore $B=\emptyset$.

$(3) \Rightarrow(1)$ If there are no nonempty open bisections contained in Iso $(\mathcal{G}) \backslash \mathcal{G}^{(0)}$, then there are no nonempty open subsets of $\operatorname{Iso}(\mathcal{G}) \backslash \mathcal{G}^{(0)}$, and therefore $\operatorname{Iso}(\mathcal{G}) \backslash \mathcal{G}^{(0)}$ has empty interior.

$(3) \Rightarrow(4)$ We begin by proving a claim: if $B \subseteq \mathcal{G} \backslash \mathcal{G}^{(0)}$ is an open bisection and $U \subseteq \mathcal{G}^{(0)}$ is open and nonempty, then there exists a nonempty open subset $V \subseteq U$ such that $V B V=\emptyset$. If $U B U=\emptyset$, then set $U=V$ and we are done. Otherwise, $U B U \subseteq B \subseteq \mathcal{G} \backslash \mathcal{G}^{(0)}$ is a nonempty open bisection. Applying (3), there exists some $g \in U B U$ with $\boldsymbol{d}(g) \neq \boldsymbol{c}(g)$. Naturally, $\boldsymbol{d}(g), \boldsymbol{c}(g) \in U$. By the Hausdorff property, there exist disjoint open sets $W, W^{\prime} \subseteq U$ with $\boldsymbol{c}(g) \in W$ and $\boldsymbol{d}(g) \in W^{\prime}$. Set $V=W \cap \boldsymbol{c}\left(B W^{\prime}\right)$. Then $c(g) \in V$, so $V$ is nonempty, and

$$
V B=\left(W \cap \boldsymbol{c}\left(B W^{\prime}\right)\right) B=W B \cap \boldsymbol{c}\left(B W^{\prime}\right) B=W B \cap B W^{\prime} .
$$

The last equality uses the fact that $B$ is a bisection, so $c\left(B W^{\prime}\right) B=B W^{\prime}$. Therefore,

$$
V B V=\left(W B \cap B W^{\prime}\right) V \subseteq\left(B W^{\prime}\right) V \subseteq\left(B W^{\prime}\right) W=\emptyset,
$$

because $W^{\prime} W=W^{\prime} \cap W=\emptyset$. This proves the claim.

Now, let $K \subseteq \mathcal{G} \backslash \mathcal{G}^{(0)}$ be a compact set, and let $U \subseteq \mathcal{G}^{(0)}$ be open and nonempty. We set out to construct a nonempty open subset $V \subseteq U$ such that $V K V=\emptyset$. The set $K$, being compact, can be covered by finitely many open bisections: $K \subseteq B_{1} \cup \cdots \cup B_{n}$. The claim in the previous paragraph proves the existence of a nonempty open set $V_{1} \subseteq U$, such that $V_{1} B_{1} V_{1}=\emptyset$. Similarly, there is a nonempty open $V_{2} \subseteq V_{1}$ such that $V_{2} B_{2} V_{2}=\emptyset$. Inductively, this produces a chain of open sets $\emptyset \neq V_{n} \subseteq V_{n-1} \subseteq \cdots \subseteq V_{1} \subseteq U$ such that $V_{i} B_{i} V_{i}=\emptyset$ for $1 \leq i \leq n$. Setting $V=V_{n}$, we have

$$
V K V \subseteq V\left(B_{1} \cup \cdots \cup B_{n}\right) V \subseteq V_{1} B_{1} V_{1} \cup \cdots \cup V_{n} B_{n} V_{n}=\emptyset .
$$

(4) $\Rightarrow(3)$ Suppose (3) does not hold, so there is a nonempty open bisection $B_{0} \subseteq \mathcal{G} \backslash \mathcal{G}^{(0)}$ with $B_{0} \subseteq$ Iso $(\mathcal{G})$. By shrinking it if necessary, we can assume $B_{0}$ is precompact. Let $K_{0}=\overline{B_{0}}$, the closure of $B_{0}$. As Iso $(\mathcal{G})$ is closed in $\mathcal{G}$, we have that $K_{0} \subseteq \operatorname{Iso}(\mathcal{G})$. Let $U_{0}=\boldsymbol{c}\left(B_{0}\right)$ and take any $\emptyset \neq V \subseteq U_{0}$. Since $K_{0} \subseteq \operatorname{Iso}(\mathcal{G})$, it follows that $V K_{0}=K_{0} V \neq \emptyset$, so $V K_{0} V \neq \emptyset$. Therefore (4) does not hold, because there is no $V \subseteq U_{0}$ such that $V K_{0} V=\emptyset$.

Remark 3.19. The original proof of the "(3) $\Rightarrow(4)$ " part of [19, Lemma 3.1], does not appear to be correct. There are examples for which the set $V$ defined in the proof is empty. Fortunately, this problem is resolved by defining $V$ inductively, as we have done in the proof of Lemma 3.18.

Lemma 3.20. [60, Proposition 3.6 (i)] If a Hausdorff étale groupoid $\mathcal{G}$ is topologically principal, then it is effective.

Proof. Suppose $\mathcal{G}$ is topologically principal: the set $D=\left\{x \in \mathcal{G}^{(0)} \mid{ }^{x} \mathcal{G}^{x}=\{x\}\right\}$ is dense in $\mathcal{G}^{(0)}$. If $U \subseteq \operatorname{Iso}(\mathcal{G}) \backslash \mathcal{G}^{(0)}$ is an open bisection (i.e., open in $\mathcal{G}$ ) then $\boldsymbol{d}(U)$ is an open subset of $\mathcal{G}^{(0)} \backslash D$, but $D$ is dense in $\mathcal{G}^{(0)}$, so $\boldsymbol{d}(U)=\emptyset$, which implies $U=\emptyset$. This proves Iso $(\mathcal{G}) \backslash \mathcal{G}^{(0)}$ has empty interior, which implies $\mathcal{G}$ is effective (noting that the proof of $(1) \Rightarrow(2)$ in Lemma 3.18 only requires $\mathcal{G}$ to be Hausdorff and étale).

The following result is an analogue of [2, Corollary 2.2.13], and it is just an alternative way of presenting some content from [24] and [64].

Proposition 3.21. Let $\mathcal{G}$ be a $\Gamma$-graded Hausdorff ample groupoid such that $\mathcal{G}_{\varepsilon}$ is effective. Given a nonzero homogeneous element $h \in A_{R}(\mathcal{G})_{\gamma}$, there exists $C \in B_{\gamma^{-1}}^{\text {co }}(\mathcal{G})$, nonempty $V \in \mathcal{B}\left(\mathcal{G}^{(0)}\right)$, and nonzero $r \in R$ such that $\mathbf{1}_{C} * h * \mathbf{1}_{V}=r \mathbf{1}_{V}$. 
Proof. Step 1 [24, Lemma 3.1]: We show that there exists $B \in B_{\gamma^{-1}}^{\text {co }}(\mathcal{G})$ such that the function $f=\mathbf{1}_{B} * h$ is $\varepsilon$-homogeneous and its support has nonempty intersection with $\mathcal{G}^{(0)}$. Applying Lemma 1.34, we can write $h=\sum_{i=1}^{n} r_{i} \mathbf{1}_{D_{i}}$, where $r_{1}, \ldots, r_{n} \in R \backslash\{0\}$ and $D_{1}, \ldots, D_{n} \in B_{*}^{\text {co }}(\mathcal{G})$ are mutually disjoint. Since the $D_{i}$ are disjoint and the $r_{i}$ are nonzero, we can assume each $D_{i} \subseteq \mathcal{G}_{\gamma}$. Let $B=D_{1}^{-1}$ and define $f=\mathbf{1}_{B} * h$. Then

$$
f=\mathbf{1}_{B} * h=\sum_{i=1}^{n} r_{i} \mathbf{1}_{B} * \mathbf{1}_{D_{i}}=\sum_{i=1}^{n} r_{i} \mathbf{1}_{B D_{i}}=r_{1} \mathbf{1}_{B B^{-1}}+\sum_{i=2}^{n} r_{i} \mathbf{1}_{B D_{i}} \in A_{R}(\mathcal{G})_{\varepsilon} .
$$

Note that $B D_{1}, \ldots, B D_{n} \in B_{\varepsilon}^{\mathrm{co}}(\mathcal{G})$ are mutually disjoint. Indeed, if $x \in B$ and $y \in D_{i}$ are composable, then $x y \in B D_{j}$ implies $y=x^{-1} x y \in B^{-1} B D_{j}=\boldsymbol{d}(B) D_{j} \subseteq D_{j}$. But $y \in D_{i} \cap D_{j}$ implies $i=j$ because $D_{1}, \ldots, D_{n}$ are disjoint. To show that $(\operatorname{supp} f) \cap \mathcal{G}^{(0)} \neq \emptyset$, let $x \in B$. Then $x x^{-1} \in B D_{i}$ if and only if $i=1$. Consequently, $f\left(x x^{-1}\right)=r_{1} \neq 0$, so $x x^{-1} \in(\operatorname{supp} f) \cap \mathcal{G}^{(0)}$.

Step 2 [24, 64]: We show that there exists $V \in \mathcal{B}\left(\mathcal{G}^{(0)}\right)$ such that $\mathbf{1}_{V} * f * \mathbf{1}_{V}=r_{1} \mathbf{1}_{V}$, where $f$ is from Step 1. The set $K=(\operatorname{supp} f) \backslash B B^{-1}=B D_{2} \cup \cdots \cup B D_{n}$ is a compact subset of $\mathcal{G}_{\varepsilon} \backslash \mathcal{G}^{(0)}$. Since $\mathcal{G}_{\varepsilon}$ is effective, Lemma 3.18 (4) proves that a nonempty open set $V \subseteq B B^{-1}=c(B)$ exists such that $V K V=\emptyset$. By shrinking if necessary, we can assume $V$ is compact. This yields

$$
\mathbf{1}_{V} * f * \mathbf{1}_{V}=r_{1} \mathbf{1}_{V\left(B B^{-1}\right) V}+\sum_{i=2}^{n} r_{i} \mathbf{1}_{V\left(B D_{i}\right) V}=r_{1} \mathbf{1}_{V}
$$

For completion: set $C=V B$ and $r=r_{1}$. Then $C \in B_{\gamma^{-1}}^{\text {co }}(\mathcal{G}), V \in \mathcal{B}\left(\mathcal{G}^{(0)}\right)$ is nonempty, $r \in R$ is nonzero, and $\mathbf{1}_{C} * h * \mathbf{1}_{V}=\mathbf{1}_{V} * \mathbf{1}_{B} * h * \mathbf{1}_{V}=\mathbf{1}_{V} * f * \mathbf{1}_{V}=r \mathbf{1}_{V}$.

We are now in a position to prove the Graded Uniqueness Theorem for Steinberg algebras.

Theorem 3.22 (Graded Uniqueness Theorem for Steinberg algebras). [24, Theorem 3.4]

Let $\mathcal{G}$ be a $\Gamma$-graded Hausdorff ample groupoid such that $\mathcal{G}_{\varepsilon}$ is effective. If $A$ is a $\Gamma$-graded ring and $\phi: A_{R}(\mathcal{G}) \rightarrow A$ is a graded homomorphism with the property that $\phi\left(r \mathbf{1}_{V}\right) \neq 0$ for every nonempty $V \in \mathcal{B}\left(\mathcal{G}^{(0)}\right)$ and every $r \in R \backslash\{0\}$, then $\phi$ is injective.

Proof. The kernel of $\phi$ is a graded ideal. Let $h \in(\operatorname{ker} \phi)_{\gamma}$. If $h \neq 0$ then, according to Proposition 3.21 , there exists a compact open bisection $C \subseteq \mathcal{G}_{\gamma^{-1}}$ and a nonempty compact open set $V \subseteq \mathcal{G}^{(0)}$ such that $\mathbf{1}_{C} * h * \mathbf{1}_{V}=r \mathbf{1}_{V}$ for some $r \neq 0$. Then $\phi\left(r \mathbf{1}_{V}\right)=\phi\left(\mathbf{1}_{C}\right) \phi(h) \phi\left(\mathbf{1}_{V}\right)=0$, which contradicts the assumption about $\phi$. Therefore $h=0$, so $(\operatorname{ker} \phi)_{\gamma}=0$. Since this is true for every $\gamma \in \Gamma$, $\operatorname{ker} \phi=$ $\bigoplus_{\gamma \in \Gamma}(\operatorname{ker} \phi)_{\gamma}=0$.

Remark 3.23. If $\mathcal{G}=\mathcal{G}_{E}$ is the groupoid of a graph $E$, then

$$
\mathcal{G}_{0}=\bigcup\left\{\mathcal{Z}(\alpha, \beta)\left|(\alpha, \beta) \in E^{\star} \times_{r} E^{\star},\right| \alpha|=| \beta \mid\right\}
$$

so $\operatorname{Iso}\left(\mathcal{G}_{0}\right)=\operatorname{Iso}\left(\mathcal{G}_{0}\right)^{\circ}=\mathcal{G}^{(0)}$, which shows that $\mathcal{G}$ satisfies the hypotheses of Theorem 3.22. The Graded Uniqueness Theorem for Steinberg algebras is a generalisation of the Graded Uniqueness Theorem for Leavitt path algebras, notwithstanding the fact that the latter theorem is usually called upon to prove that all Leavitt path algebras are Steinberg algebras.

Any groupoid can be graded by the trivial group $\{\varepsilon\}$. With this observation, we immediately obtain the Cuntz-Krieger Uniqueness Theorem for Steinberg algebras [24, Theorem 3.2] .

Corollary 3.24 (Cuntz-Krieger Uniqueness Theorem for Steinberg algebras).

Let $\mathcal{G}$ be an effective Hausdorff ample groupoid. If $A$ is a ring and $\phi: A_{R}(\mathcal{G}) \rightarrow A$ is a homomorphism with the property that $\phi\left(r \mathbf{1}_{V}\right) \neq 0$ for every nonempty $V \in \mathcal{B}\left(\mathcal{G}^{(0)}\right)$ and every $r \in R \backslash\{0\}$, then $\phi$ is injective.

We now show how Condition (L) translates to the groupoid setting. 
Proposition 3.25. If $E$ is a graph, then $\mathcal{G}_{E}$ is effective if and only if $\mathcal{G}_{E}$ is topologically principal, if and only if E satisfies Condition ( $L$ ).

Proof. [67] Assume that $E$ satisfies Condition (L), so that every closed path has an exit. Then every basic open set in $\partial E$ contains a path that is not eventually periodic. Such paths have trivial isotropy groups in $\mathcal{G}$, by Proposition 2.12, so $\mathcal{G}^{(0)}$ has a dense subset with trivial isotropy. This implies $\mathcal{G}$ is topologically principal, hence effective, by Lemma 3.20. On the other hand, if $E$ does not satisfy Condition $(\mathrm{L})$, then there exists a cycle $c$ without an exit, and $\mathcal{G}_{E}$ is not effective because there is an open set: $\mathcal{Z}(c c,|c|, c)=\{(c c c \ldots,|c|, c c c \ldots)\} \subseteq \operatorname{Iso}(\mathcal{G}) \backslash \mathcal{G}^{(0)}$.

Having proved the Cuntz-Krieger Uniqueness Theorem for Steinberg algebras, we can prove the CuntzKrieger Uniqueness Theorem for Leavitt path algebras (see Theorem 3.13), once and for all, in its full generality.

Theorem 3.26 (Cuntz-Krieger Uniqueness Theorem for Leavitt path algebras).

Let $E$ be a graph satisfying Condition $(L)$ and let $R$ be a unital commutative ring. If $A$ is a ring and $\psi: L_{R}(E) \rightarrow A$ is a homomorphism with the property that $\psi(r v) \neq 0$ for every $v \in E^{0}$ and every $r \in R \backslash\{0\}$, then $\psi$ is injective.

Proof. First of all, suppose $r \in R \backslash\{0\}, \mu \in E^{\star}$, and $F$ is a finite proper subset of $r(\mu) E^{1}$. Let $x=r \mu \mu^{*}-r \sum_{e \in F} \mu e e^{*} \mu^{*}$. Then $0 \neq x \in L_{R}(E)_{0}$, so Lemma 3.9 yields $(\alpha, \beta) \in E^{\star} \times_{r} E^{\star}, v \in E^{0}$, and $s \in R \backslash\{0\}$ such that $\alpha^{*} x \beta=s v$. This implies that $\psi\left(\alpha^{*}\right) \psi(x) \psi(\beta)=\psi(s v) \neq 0$, so $\psi(x) \neq 0$.

By Proposition 3.25, the groupoid $\mathcal{G}_{E}$ is effective. Let $\phi: A_{R}\left(\mathcal{G}_{E}\right) \rightarrow A$ be the map $\phi=\psi \circ \pi^{-1}$, where $\pi: L_{R}(E) \rightarrow A_{R}\left(\mathcal{G}_{E}\right)$ is the isomorphism from Theorem 3.14. Suppose $V \subseteq \partial E$ is compact and open, and $r \in R \backslash\{0\}$. We can find $\mu \in E^{\star}$ and $F \subseteq_{\text {finite }} r(\mu) E^{1}$ such that $Z(\mu, F)$ is a nonempty open subset of $V$. Then $Z(\mu, F) V=Z(\mu, F) \cap V=Z(\mu, F)$, so $r \mathbf{1}_{Z(\mu, F)}=\mathbf{1}_{Z(\mu, F)} * r \mathbf{1}_{V}$. Noting that $\pi^{-1}\left(r \mathbf{1}_{Z(\mu, F)}\right)=r \mu \mu^{*}-r \sum_{e \in F} \mu e e^{*} \mu^{*}$, the first paragraph proves that $0 \neq \psi \circ \pi^{-1}\left(r \mathbf{1}_{Z(\mu, F)}\right)=$ $\phi\left(r \mathbf{1}_{Z(\mu, F)}\right)=\phi\left(\mathbf{1}_{\mathcal{Z}(\mu, F)}\right) \phi\left(r \mathbf{1}_{V}\right)$; consequently $\phi\left(r \mathbf{1}_{V}\right) \neq 0$. Applying Corollary 3.24 , the map $\phi$ is injective. Conclude that $\psi=\phi \circ \pi$ is injective.

\section{AcKNOWLEDGEMENTs}

I thank Juana Sánchez Ortega, for her valuable advice and guidance throughout my Masters degree. I also thank Tran Giang Nam for finding an important error in an earlier version of Theorem 2.4, and suggesting a way to fix it. (Any errors that remain are my own responsibility.) Finally, I thank the two examiners of my Masters thesis, Pere Ara and Aidan Sims, who wrote very insightful comments that led to an improvement of this work.

I acknowledge the support of the National Research Foundation of South Africa.

\section{REFERENCES}

[1] Abrams, G. Leavitt path algebras: the first decade. Bulletin of Mathematical Sciences 5, 1 (2015), 59-120.

[2] Abrams, G., Ara, P., And Siles Molina, M. Leavitt path algebras, vol. 2191 of Lecture Notes in Mathematics. Springer, 2017.

[3] Abrams, G., and Aranda Pino, G. The Leavitt path algebra of a graph. Journal of Algebra 293, 2 (2005), 319-334.

[4] Abrams, G., and Aranda Pino, G. The Leavitt path algebras of arbitrary graphs. Houston Journal of Mathematics 34, 2 (2008), 423-442.

[5] Abrams, G., Aranda Pino, G., and Siles Molina, M. Finite-dimensional Leavitt path algebras. Journal of Pure and Applied Algebra 209, 3 (2007), 753-762.

[6] Abrams, G., Bell, J., and Rangaswamy, K. M. On prime nonprimitive von Neumann regular algebras. Transactions of the American Mathematical Society 366, 5 (2014), 2375-2392.

[7] Abrams, G., And Tomforde, M. Isomorphism and Morita equivalence of graph algebras. Transactions of the American Mathematical Society 363, 7 (2011), 3733-3767. 
8] Alahmedi, A., Alsulami, H., Jain, S., And Zelmanov, E. I. Structure of Leavitt path algebras of polynomial growth. Proceedings of the National Academy of Sciences of the United States of America 110, 38 (2013), 15222-15224.

[9] Ambily, A. A., Hazrat, R., And Li, H. Simple flat Leavitt path algebras are von Neumann regular. Communications in Algebra, to appear (2018).

[10] Ánh, P., AND Márki, L. Morita equivalence for rings without identity. Tsukuba Journal of Mathematics 11, 1 (1987), $1-16$.

[11] Ara, P., Bosa, J., Hazrat, R., and Sims, A. Reconstruction of graded groupoids from graded Steinberg algebras Forum Mathematicum 29, 5 (2017), 1023-1037.

[12] Ara, P., Goodearl, K. R., And Pardo, E. $K_{0}$ of purely infinite simple regular rings. K-theory 26, 1 (2002), 69-100.

[13] Ara, P., Moreno, M. A., And Pardo, E. Nonstable $K$-theory for graph algebras. Algebras and Representation Theory 10, 2 (2007), 157-178.

[14] Aranda Pino, G., Martín Barquero, D., Martín González, C., and Siles Molina, M. Socle theory for Leavitt path algebras of arbitrary graphs. Revista Matemática Iberoamericana 26, 2 (2010), 611-638.

[15] Aranda Pino, G., Rangaswamy, K. M., And Vaš, L. *-Regular Leavitt path algebras of arbitrary graphs. Acta Mathematica Sinica, English Series 28, 5 (2012), 957-968.

[16] Bates, T., Pask, D., Raeburn, I., and Szymański, W. The $C^{*}$-algebras of row-finite graphs. New York Journal of Mathematics 6, 307 (2000), 324.

[17] Beuter, V., And Gonçalves, D. The interplay between Steinberg algebras and skew group rings. Journal of Algebra $487(2018)$

[18] BreŠAR, M. Introduction to Noncommutative Algebra. Universitext. Springer, 2014.

[19] Brown, J., Clark, L. O., Farthing, C., And Sims, A. Simplicity of algebras associated to étale groupoids. Semigroup Forum 88, 2 (2014), 433-452.

[20] Brown, J. H., Clark, L. O., and an Huef, A. Diagonal-preserving ring $*$-isomorphisms of Leavitt path algebras. Journal of Pure and Applied Algebra 221, 10 (2017), 2458-2481.

[21] Brownlowe, N., Carlsen, T. M., and Whittaker, M. F. Graph algebras and orbit equivalence. Ergodic Theory and Dynamical Systems 37, 2 (2017), 389-417.

[22] Carlsen, T. M. *-isomorphism of Leavitt path algebras over $\mathbb{Z}$. Advances in Mathematics 324 (2018), 326-335.

[23] Carlsen, T. M., and Rout, J. Diagonal-preserving graded isomorphisms of Steinberg algebras. Communications in Contemporary Mathematics (2017), 1750064.

[24] Clark, L. O., And Edie-Michell, C. Uniqueness theorems for Steinberg algebras. Algebras and Representation Theory 18, 4 (2015), 907-916.

[25] Clark, L. O., Exel, R., and Pardo, E. A generalized uniqueness theorem and the graded ideal structure of Steinberg algebras. Forum Mathematicum 30, 3 (2018), 533-552.

[26] Clark, L. O., Exel, R., Pardo, E., Sims, A., and Starling, C. Simplicity of algebras associated to non-Hausdorff groupoids. arXiv preprint, arXiv:1806.04362v1 (2018).

[27] Clark, L. O., Farthing, C., Sims, A., and Tomforde, M. A groupoid generalisation of Leavitt path algebras. Semigroup Forum 89, 3 (2014), 501-517.

[28] Clark, L. O., Martín Barquero, D., Martín González, C., and Siles Molina, M. Using Steinberg algebras to study decomposability of Leavitt path algebras. Forum Mathematicum 29, 6 (2016), 1311-1324.

29] Clark, L. O., Martín Barquero, D., Martín González, C., and Siles Molina, M. Using the Steinberg algebra model to determine the center of any Leavitt path algebra. Israel Journal of Mathematics, to appear (2018), 1-22.

[30] Clark, L. O., and Pangalela, Y. E. Cohn path algebras of higher-rank graphs. Algebras and Representation Theory 20, 1 (2017), 47-70.

[31] Clark, L. O., and Pangalela, Y. E. Kumjian-Pask algebras of finitely aligned higher-rank graphs. Journal of Algebra $482(2017), 364-397$

[32] Clark, L. O., And Sims, A. Equivalent groupoids have Morita equivalent Steinberg algebras. Journal of Pure and Applied Algebra 219, 6 (2015), 2062-2075.

[33] Cuntz, J. Simple $C^{*}$-algebras generated by isometries. Communications in Mathematical Physics 57, 2 (1977), 173185.

[34] Cuntz, J., And Krieger, W. A class of $C^{*}$-algebras and topological Markov chains. Inventiones mathematicae 56, 3 (1980), 251-268.

[35] Deaconu, V. Groupoids associated with endomorphisms. Transactions of the American Mathematical Society 347, 5 (1995), 1779-1786.

[36] Dokuchaev, M., Exel, R., And Piccione, P. Partial representations and partial group algebras. Journal of Algebra 226, 1 (2000), 505-532.

[37] Eilers, S., Restorff, G., Ruiz, E., And Sørensen, A. P. The complete classification of unital graph $C^{*}$-algebras: Geometric and strong. arXiv preprint, arXiv:1611.07120v1 (2016).

[38] Exel, R. Inverse semigroups and combinatorial $C^{*}$-algebras. Bulletin of the Brazilian Mathematical Society, New Series 39, 2 (2008), 191-313.

[39] Exel, R. Reconstructing a totally disconnected groupoid from its ample semigroup. Proceedings of the American Mathematical Society 138, 8 (2010), 2991-3001.

[40] Exel, R. Partial Dynamical Systems, Fell Bundles and Applications, vol. 224 of Mathematical Surveys and Monographs. American Mathematical Society, 2017.

[41] Gonçalves, D., And Royer, D. Leavitt path algebras as partial skew group rings. Communications in Algebra 42, 8 (2014), 3578-3592 
[42] Goodearl, K. R. Von Neumann Regular Rings. Pitman, 1979.

[43] Goodearl, K. R. Leavitt path algebras and direct limits. Contemporary Mathematics 480, 200 (2009), $165-187$.

[44] Hazrat, R. The graded Grothendieck group and the classification of Leavitt path algebras. Mathematische Annalen 355, 1 (2013), 273-325.

[45] Hazrat, R., And Li, H. Graded Steinberg algebras and partial actions. Journal of Pure and Applied Algebra 222, 12 (2018), 3946-3967.

[46] Hazrat, R., And Vaš, L. Baer and Baer *-ring characterizations of Leavitt path algebras. Journal of Pure and Applied Algebra 222, 1 (2018), 39-60.

[47] Kanuni, M., Martín Barquero, D., Martín González, C., And Siles Molina, M. Classification of Leavitt path algebras with two vertices. arXiv preprint, arXiv:1708.03128v2 (2017).

[48] Kaplansky, I. Fields and Rings, 2nd ed. Chicago Lectures in Mathematics. University of Chicago Press, 1972.

[49] Kumjian, A., And Pask, D. Higher rank graph $C^{*}$-algebras. New York Journal of Mathematics 6, 1 (2000), 1-20.

[50] Kumjian, A., Pask, D., Raeburn, I., And Renault, J. Graphs, groupoids, and Cuntz-Krieger algebras. Journal of Functional Analysis 144, 2 (1997), 505-541.

[51] Leavitt, W. G. The module type of a ring. Transactions of the American Mathematical Society 103, 1 (1962), 113-130.

[52] Leavitt, W. G. The module type of homomorphic images. Duke Mathematical Journal 32, 2 (1965), 305-311.

[53] Loday, J.-L. Cyclic Homology, 2nd ed., vol. 301 of Grundlehren der mathematischen Wissenschaften. Springer, 1998.

[54] NekrashevyCh, V. Growth of étale groupoids and simple algebras. International Journal of Algebra and Computation 26, 2 (2016), 375-397.

[55] PArdo, E. The isomorphism problem for Higman-Thompson groups. Journal of Algebra 344, (2011), 172-183.

[56] Paterson, A. L. T. Groupoids, Inverse Semigroups, and their Operator Algebras, vol. 170 of Progress in Mathematics, Springer, 1999.

[57] Paterson, A. L. T. Graph inverse semigroups, groupoids and their $C^{*}$-algebras. Journal of Operator Theory 48, 3 (2002), 645-662.

[58] Raeburn, I. Graph Algebras, vol. 103 of CBMS Regional Conference Series in Mathematics. American Mathematical Society, 2005.

[59] Renault, J. A Groupoid Approach to $C^{*}$-Algebras, vol. 793 of Lecture Notes in Mathematics. Springer-Verlag, 1980.

[60] Renault, J. Cartan subalgebras in $C^{*}$-algebras. Bulletin of the Irish Mathematical Society, 61 (2008), 29-63.

[61] Renault, J., Sims, A., Williams, D., And Yeend, T. Uniqueness theorems for topological higher-rank graph $C^{*}$ algebras. Proceedings of the American Mathematical Society 146, 2 (2018), 669-684.

[62] Sims, A. Étale groupoids and their $C^{*}$-algebras. arXiv preprint, arXiv:1710.10897v1 (2017).

[63] SteinberG, B. A groupoid approach to discrete inverse semigroup algebras. Advances in Mathematics 223, 2 (2010), $689-727$.

[64] SteinberG, B. Simplicity, primitivity and semiprimitivity of étale groupoid algebras with applications to inverse semigroup algebras. Journal of Pure and Applied Algebra 220, 3 (2016), 1035-1054.

[65] Steinberg, B. Chain conditions on étale groupoid algebras with applications to Leavitt path algebras and inverse semigroup algebras. Journal of the Australian Mathematical Society 104, 3 (2018), 403-411.

[66] Steinberg, B. Diagonal-preserving isomorphisms of étale groupoid algebras. Journal of Algebra, to appear (2018).

[67] Steinberg, B. Prime étale groupoid algebras with applications to inverse semigroup and Leavitt path algebras. Journal of Pure and Applied Algebra, to appear (2018).

[68] Tomforde, M. Leavitt path algebras with coefficients in a commutative ring. Journal of Pure and Applied Algebra 215, 4 (2011), 471-484.

[69] Webster, S. The path space of a directed graph. Proceedings of the American Mathematical Society 142, 1 (2014), $213-225$

[70] Willard, S. General Topology. Addison-Wesley Series in Mathematics. Addison-Wesley, 1970.

[71] YeEnd, T. Groupoid models for the $C^{*}$-algebras of topological higher-rank graphs. Journal of Operator Theory 57, 1 (2007), 95-120.

Department of Mathematics and Applied Mathematics, University of Cape Town, South Africa.

Department of Mathematics: Algebra and Geometry, Ghent University, Belgium.

E-mail address: simon.rigby@ugent.be 